\title{
DEGREE AND ALGEBRAIC PROPERTIES OF LATTICE AND MATRIX IDEALS*
}

\author{
LIAM O'CARROLL ${ }^{\dagger}$, FRANCESC PLANAS-VILANOVA $^{\ddagger}$, AND RAFAEL H. VILLARREAL ${ }^{\S}$
}

\begin{abstract}
We study the degree of nonhomogeneous lattice ideals over arbitrary fields, and give formulas to compute the degree in terms of the torsion of certain factor groups of $\mathbb{Z}^{s}$ and in terms of relative volumes of lattice polytopes. We also study primary decompositions of lattice ideals over an arbitrary field using the Eisenbud-Sturmfels theory of binomial ideals over algebraically closed fields. We then use these results to study certain families of integer matrices (positive critical binomial (PCB), generalized positive critical binomial (GPCB), critical binomial (CB), and generalized critical binomial (GCB) matrices) and the algebra of their corresponding matrix ideals. In particular, the family of GPCB matrices is shown to be closed under transposition, and previous results for PCB ideals are extended to GPCB ideals. Then, more particularly, we give some applications to the theory of 1-dimensional binomial ideals. If $G$ is a connected graph, we show as a further application that the order of its sandpile group is the degree of the Laplacian ideal and the degree of the toppling ideal. We also use our earlier results to give a structure theorem for graded lattice ideals of dimension 1 in 3 variables and for homogeneous lattices in $\mathbb{Z}^{3}$ in terms of $\mathrm{CB}$ ideals and $\mathrm{CB}$ matrices, respectively, thus complementing a well-known theorem of Herzog on the toric ideal of a monomial space curve.
\end{abstract}

Key words. lattice ideal, graded binomial ideal, degree, primary decomposition, PCB ideal

AMS subject classifications. 13F20, 13A15, 13H15, 13P05, 05E40, 14 Q99

DOI. $10.1137 / 130922094$

1. Introduction. Let $S=K\left[t_{1}, \ldots, t_{s}\right]$ be a polynomial ring over a field $K$. As usual, $\mathfrak{m}$ will denote the maximal ideal of $S$ generated by $t_{1}, \ldots, t_{s}$. For an arbitrary ideal $I$ of $S$ there are various ways of introducing the notion of degree; let us briefly recall one of them. The vector space of polynomials in $S$ (resp., $I$ ) of degree at most $i$ is denoted by $S_{\leq i}\left(\operatorname{resp} ., I_{\leq i}\right)$. If $H_{I}^{a}(i):=\operatorname{dim}_{K}\left(S_{\leq i} / I_{\leq i}\right)$ is the affine Hilbert function of $S / I$ and $k$ is the Krull dimension of $S / I$, the positive integer

$$
\operatorname{deg}(S / I):=k ! \lim _{i \rightarrow \infty} H_{I}^{a}(i) / i^{k}
$$

is called the degree or multiplicity of $S / I$. If $S=\oplus_{i=0}^{\infty} S_{i}$ has the standard grading and $I \subset S$ is a graded ideal, then $H_{I}^{a}$ is the Hilbert-Samuel function of $S / I$ with respect to $\mathfrak{m}$ in the sense of [35, Definition B.3.1]. The notion of degree plays a central role in this paper. One of our aims is to give a formula for the degree when $I$ is any lattice ideal.

The set of nonnegative integers (resp., positive integers) is denoted by $\mathbb{N}$ (resp., $\mathbb{N}_{+}$). A binomial is a polynomial of the form $t^{b}-t^{c}$, where $b, c \in \mathbb{N}^{s}$ and where, if $c=\left(c_{i}\right) \in \mathbb{N}^{s}$, we set $t^{c}=t_{1}^{c_{1}} \cdots t_{s}^{c_{s}}$. We use the term "binomial" as a shorthand for what elsewhere has been called pure difference binomial $[10$, p. 2] or unital binomial

${ }^{*}$ Received by the editors May 24, 2013; accepted for publication (in revised form) January 6, 2014; published electronically March 20, 2014.

http://www.siam.org/journals/sidma/28-1/92209.html

${ }^{\dagger}$ Maxwell Institute for Mathematical Sciences, School of Mathematics, University of Edinburgh, EH9 3JZ, Edinburgh, Scotland (L.O'Carroll@ed.ac.uk).

${ }^{\ddagger}$ Departament de Matemàtica Aplicada 1, Universitat Politècnica de Catalunya, Diagonal 647, ETSEIB, 08028 Barcelona, Catalunya (francesc.planas@upc.edu). This author's research was partially supported by the MTM2010-20279-C02-01 grant.

$\S$ Departamento de Matemáticas, Centro de Investigación y de Estudios Avanzados del IPN, Apartado Postal 14-740, 07000 Mexico City, Mexico (vila@math.cinvestav.mx). This author's research was partially supported by SNI. 
[18]. A binomial ideal is an ideal generated by binomials. The set $\left\{i \mid c_{i} \neq 0\right\}$, denoted by $\operatorname{supp}(c)$, is called the support of $c$.

Consider an $s \times m$ integer matrix $L$ with column vectors $a_{1}, \ldots, a_{m}$. Each $a_{i}$ can be written uniquely as $a_{i}=a_{i}^{+}-a_{i}^{-}$, where $a_{i}^{+}$and $a_{i}^{-}$are in $\mathbb{N}^{s}$ and have disjoint support. The matrix ideal of $L$, denoted by $I(L)$, is the ideal of $S$ generated by $t^{a_{1}^{+}}-t^{a_{1}^{-}}, \ldots, t_{m}^{a_{m}^{+}}-t^{a_{m}^{-}}$. A matrix ideal is an ideal of the form $I(L)$ for some $L$. Matrix ideals are a special class of binomial ideals. Some of our results concern certain integer matrices and the algebra of their matrix ideals.

A subgroup $\mathcal{L}$ of $\mathbb{Z}^{s}$ is called a lattice. A lattice ideal, over the field $K$, is an ideal of $S$ of the form $I(\mathcal{L})=\left(t^{a^{+}}-t^{a^{-}} \mid a \in \mathcal{L}\right)$ for some lattice $\mathcal{L}$ in $\mathbb{Z}^{s}$. Let $\mathcal{L}$ be the lattice generated by the columns $a_{1}, \ldots, a_{m}$ of an integer matrix $L$. It is well known that $I(L)$ and $I(\mathcal{L})$ are related by the equality $I(\mathcal{L})=\left(I(L):\left(t_{1} \cdots t_{s}\right)^{\infty}\right)$ and that $I(\mathcal{L})$ is also a matrix ideal. The class of lattice ideals has been studied in many places; see [27] and the references therein. This concept is a natural generalization of a toric ideal.

Using commutative algebra methods and the Eisenbud-Sturmfels theory of binomial ideals over algebraically closed fields [10], in this paper we study algebraic properties and primary decompositions of lattice ideals and binomial ideals of a variety of types. By and large, we focus on the structure of the class of graded binomial ideals $I$ that satisfy the vanishing condition $V\left(I, t_{i}\right)=\{0\}$ for all $i$, where $V(\cdot)$ is the variety of $(\cdot)$. This class of ideals includes the graded lattice ideals of dimension 1 [20], the vanishing ideals over finite fields of algebraic toric sets [33], the toric ideals of monomial curves [16], the Herzog-Northcott ideals [29], the positive critical binomial (PCB) ideals [30], and the Laplacian ideals of complete graphs [23]. We will also present some other interesting families of ideals that satisfy this hypothesis. In particular, for $s=3$, we completely determine the structure of any graded lattice ideal in terms of critical binomial ideals and also the structure of any homogeneous lattice in $\mathbb{Z}^{3}$. The transpose of $L$ is denoted by $L^{\top}$. If $I\left(L^{\top}\right)$ is graded, following [30], we study when $I(L)$ is also graded.

The contents of this paper are as follows. In section 2, we introduce the notion of degree via Hilbert polynomials and observe that the degree is independent of the base field $K$ (Proposition 2.8). We present some of the results on lattice ideals that will be needed throughout the paper, introduce some notation, and recall how the structure of $T\left(\mathbb{Z}^{s} / \mathcal{L}\right)$, the torsion subgroup of $\mathbb{Z}^{s} / \mathcal{L}$, can be read off from the normal form of $L$. All results of this section are well known.

In section 3, we study primary decompositions of lattice ideals. The first main result is an auxiliary theorem that relates the degree of $S / I(\mathcal{L})$ and the torsion of $\mathbb{Z}^{s} / \mathcal{L}$ to the primary decomposition of $I(\mathcal{L})$ over an arbitrary field $K$ (Theorem 3.2). If $K$ is algebraically closed, the primary decomposition of $I(\mathcal{L})$ is given in [10, Corollary 2.5] in terms of lattice ideals of partial characters of the saturation of $\mathcal{L}$. In this situation, the primary components are generated by polynomials of the form $t^{a}-\lambda t^{b}$, where $0 \neq \lambda \in K$. Let $\gamma$ be the order of $T\left(\mathbb{Z}^{s} / \mathcal{L}\right)$. If $K$ is a field containing the $\gamma$ th roots of unity with $\operatorname{char}(K)=0$ or $\operatorname{char}(K)=p, p$ a prime with $p \nmid \gamma$, it is well known that $I(\mathcal{L})$ is a radical ideal (see Theorem 2.7). Assuming that $I(\mathcal{L})$ is a graded lattice ideal of dimension 1 , following [30] we give explicitly the minimal primary decomposition of $I(\mathcal{L})$ in terms of the normal decomposition of $L$ (Theorem 3.3).

Section 4 is devoted to developing a formula for the degree of any lattice ideal. First, we exhibit a formula for the degree that holds for any toric ideal (Theorem 4.5), the graded case was shown in [34, Theorem 4.16, p. 36] and [12]. If $S$ has the standard grading and $I(\mathcal{L})$ is a graded lattice ideal of dimension 1 , the degree of $S / I(\mathcal{L})$ is the 
order of $T\left(\mathbb{Z}^{s} / \mathcal{L}\right)$ [21]. As usual, we denote the relative volume of a lattice polytope $\mathcal{P}$ by $\operatorname{vol}(\mathcal{P})$ and the convex hull of a set $\mathcal{A}$ by $\operatorname{conv}(\mathcal{A})$.

We come to the main result of section 4 .

THEOREM 4.6.

(a) If $\operatorname{rank}(\mathcal{L})=s$, then $\operatorname{deg}(S / I(\mathcal{L}))=\left|\mathbb{Z}^{s} / \mathcal{L}\right|$.

(b) If $\operatorname{rank}(\mathcal{L})<s$, there is an integer matrix $A$ of size $(s-r) \times s$ with $\operatorname{rank}(A)=$ $s-r$ such that we have the containment of rank $r$ lattices $\mathcal{L} \subset \operatorname{ker}_{\mathbb{Z}}(A)$.

(c) If $\operatorname{rank}(\mathcal{L})<s$ and $v_{1}, \ldots, v_{s}$ are the columns of $A$, then

$$
\operatorname{deg}(S / I(\mathcal{L}))=\frac{\left|T\left(\mathbb{Z}^{s} / \mathcal{L}\right)\right|(s-r) ! \operatorname{vol}\left(\operatorname{conv}\left(0, v_{1}, \ldots, v_{s}\right)\right)}{\left|T\left(\mathbb{Z}^{s-r} /\left\langle v_{1}, \ldots, v_{s}\right\rangle\right)\right|} .
$$

One can effectively use Theorem 4.6 to compute the degree of any lattice ideal (Examples 4.11 and 4.12). For certain families, we show explicit formulas for the degree (Corollary 4.9). For a 1-dimensional lattice ideal $I(\mathcal{L})$, not necessarily homogeneous, we can express the degree in terms of a $\mathbb{Z}$-basis of the lattice $\mathcal{L}$ (Corollary 4.10, Example 8.14).

Section 5 focuses on graded binomial ideals satisfying the vanishing condition $V\left(I, t_{i}\right)=\{0\}$ for all $i$. For ideals of this type, we characterize when they are lattice ideals (Proposition 5.3). This enables us to present some applications of the main result of section 3 to the theory of binomial ideals.

We show the following result on the structure of graded matrix ideals $I$, writing $\operatorname{Hull}(I)$ for the intersection of the isolated primary components of $I$.

Proposition 5.7. Let $I$ be the matrix ideal of an $s \times m$ integer matrix $L$ and let $\mathcal{L}$ be the lattice spanned by the columns of $L$. Suppose that $I$ is graded and that $V\left(I, t_{i}\right)=\{0\}$ for all $i$. Then

(a) I has a minimal primary decomposition either of the form $I=\mathfrak{q}_{1} \cap \cdots \cap \mathfrak{q}_{c}$, if $I$ is unmixed, or else $I=\mathfrak{q}_{1} \cap \cdots \cap \mathfrak{q}_{c} \cap \mathfrak{q}$, if I is not unmixed, where the $\mathfrak{q}_{i}$ are $\mathfrak{p}_{i}$-primary ideals with $\mathrm{ht}\left(\mathfrak{p}_{i}\right)=s-1$, and $\mathfrak{q}$ is an $\mathfrak{m}$-primary ideal;

(b) $I(\mathcal{L})=\mathfrak{q}_{1} \cap \cdots \cap \mathfrak{q}_{c}=\operatorname{Hull}(I)$;

(c) $\operatorname{rank}(L)=s-1$ and there exists $\mathbf{d} \in \mathbb{N}_{+}^{s}$ with $\mathbf{d} L=0$;

(d) if $I$ is not unmixed and $h=t_{1} \cdots t_{s}$, there exists $a \in \mathbb{N}_{+}$such that $I(\mathcal{L})=$ $\left(I: h^{a}\right), \mathfrak{q}=I+\left(h^{a}\right)$ is an irredundant $\mathfrak{m}$-primary component of $I$, and $I=$ $I(\mathcal{L}) \cap \mathfrak{q} ;$

(e) either $c \leq\left|T\left(\mathbb{Z}^{s} / \mathcal{L}\right)\right|$, if $\operatorname{char}(K)=0$, or else $c \leq|G|$, if $\operatorname{char}(K)=p, p$ a prime, where $G$ is the unique largest subgroup of $T\left(\mathbb{Z}^{s} / \mathcal{L}\right)$ whose order is relatively prime to $p$. If $K$ is algebraically closed, then equality holds.

As a consequence, for the family of matrix ideals satisfying the hypotheses of Proposition 5.7, we obtain the following formula for the degree:

$$
\operatorname{deg}(S / I)=\max \left\{d_{1}, \ldots, d_{s}\right\}\left|T\left(\mathbb{Z}^{s} / \mathcal{L}\right)\right|
$$

where $\mathbf{d}=\left(d_{1}, \ldots, d_{s}\right) \in \mathbb{N}_{+}^{s}$ is the weight vector, with $\operatorname{gcd}(\mathbf{d})=1$, that makes the matrix ideal $I$ homogeneous (Corollary 5.9).

In section 6 we restrict our study to matrix ideals associated with square integer matrices of a certain type. Throughout, set $\mathbf{1}=(1, \ldots, 1)$.

Definition 1.1. Let $a_{i, j} \in \mathbb{N}, i, j=1, \ldots, s$, and let $L$ be an $s \times s$ matrix of the following special form:

$$
L=\left(\begin{array}{cccc}
a_{1,1} & -a_{1,2} & \cdots & -a_{1, s} \\
-a_{2,1} & a_{2,2} & \cdots & -a_{2, s} \\
\vdots & \vdots & \cdots & \vdots \\
-a_{s, 1} & -a_{s, 2} & \cdots & a_{s, s}
\end{array}\right) .
$$

Copyright $@$ by SIAM. Unauthorized reproduction of this article is prohibited. 
The matrix $L$ is called (a) a pure binomial matrix (PB matrix, for short) if $a_{j, j}>0$ for all $j$, and for each column of $L$ at least one off-diagonal entry is nonzero; (b) a positive pure binomial matrix (PPB matrix) if all the entries of $L$ are nonzero; (c) a critical binomial matrix (CB matrix) if $L$ is a $P B$ matrix and $L \mathbf{1}^{\top}=0$; (d) a $P C B$ matrix [30] if all the entries of $L$ are nonzero and $L \mathbf{1}^{\top}=0$; (e) a generalized critical binomial matrix (GCB matrix), if $L$ is a $P B$ matrix and there exists $\mathbf{b} \in \mathbb{N}_{+}^{s}$ such that $L \mathbf{b}^{\top}=0$; and $(f)$ a generalized positive critical binomial matrix (GPCB matrix) if all the entries of $L$ are nonzero and there exists $\mathbf{b} \in \mathbb{N}_{+}^{s}$ such that $L \mathbf{b}^{\top}=0$.

If $L$ is a PB matrix, we will call $I(L)=\left(f_{1}, \ldots, f_{s}\right)$ the $P B$ ideal associated with $L$, where $f_{i}$ is the binomial defined by the ith column of $L$ (see Definition 5.4). We will use similar terminology when $L$ is a PPB, $C B, P C B, G C B$, or GPCB matrix.

Summarizing, we have the following inclusions among these classes of matrices and ideals:

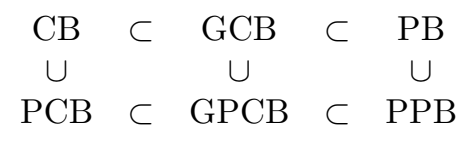

It turns out that $L$ is a $\mathrm{CB}$ matrix if and only if $L$ is the Laplacian matrix of a weighted digraph without sinks or sources (see section 7). Thus, in principle, we can and will use the techniques of algebraic graph theory [4, 14], matrix theory [3], and digraph theory [2] to study $\mathrm{CB}$ and GCB matrices and their matrix ideals.

The support of a polynomial $f$, denoted by $\operatorname{supp}(f)$, is the set of variables that occur in $f$. If $I(L)=\left(f_{1}, \ldots, f_{s}\right)$ is the matrix ideal of a PB matrix $L$ and $\left|\operatorname{supp}\left(f_{j}\right)\right| \geq$ 4 , for $j=1, \ldots, s$, we show that $I(L)$ is not a lattice ideal (Proposition 6.1). Let $g_{1}, \ldots, g_{s}$ be the binomials defined by the rows of a GCB matrix $L$ and let $I$ be the matrix ideal of $L^{\top}$. If $V\left(I, t_{i}\right)=\{0\}$ and $\left|\operatorname{supp}\left(g_{i}\right)\right| \geq 3$ for all $i$, we show that $I$ is not a complete intersection (Proposition 6.2).

The GPCB matrices (resp., ideals) are a generalization of the PCB matrices (resp., ideals) introduced and studied in [30]. The origin of this generalization is in the overlap between the results in [21, section 3] and the results in [30, section 5] (see [30, Remark 5.8]). While the class of PCB matrices is easily seen not to be closed under transposition, the wider class of GPCB matrices is shown to be closed under transposition (Theorem 6.3).

We are also interested in displaying new families of binomial ideals verifying the usual hypotheses above, namely, graded matrix ideals $I$ of integer matrices such that $V\left(I, t_{i}\right)=\{0\}$ for all $i$. Some new such families are the GPCB ideals (Remark 6.6), the Laplacian ideals associated with connected weighted graphs (Proposition 7.1), and the GCB ideals that arise from matrices with strongly connected underlying digraphs (Proposition 7.6). Thus we can apply to these families some of the results of this article.

In the rest of section 6, we extend to GPCB ideals some properties that hold for PCB ideals. We give an explicit syzygy among the generators of a GPCB ideal (Proposition 6.7). This will be used to give an explicit description of an irredundant embedded component of a GPCB ideal in at least 4 variables (Theorem 6.11). We give an explicit description of the hull of a GPCB ideal and, if $s \geq 4$, of an irredundant embedded component (Proposition 6.10 and Theorem 6.11). For $s=2$, we give a description of a GPCB ideal $I(L)$ and its hull in terms of the entries of the matrix $L$ (Lemma 6.12). This description will be used in section 8 (see Proposition 8.8) to characterize when $I(L)$ is a lattice ideal.

Copyright (c) by SIAM. Unauthorized reproduction of this article is prohibited. 
In section 7 we show how our results apply to matrix ideals arising from Laplacian matrices of weighted graphs and digraphs. We are interested in relating the combinatorics of a graph (resp., digraph) to the algebraic invariants and properties of the matrix ideal associated with the Laplacian matrix of the graph (resp., digraph). Let $G=(V, E, w)$ be a weighted simple graph, where $V=\left\{t_{1}, \ldots, t_{s}\right\}$ is the set of vertices, $E$ is the set of edges, and $w$ is a weight function that associates a weight $w_{e}$ with every $e \in E$. The Laplacian matrix of $G$, denoted by $L(G)$, is a prime example of a CB matrix. Laplacian matrices of complete graphs are PCB matrices; this type of matrix occurs in [23]. The matrix ideal $I \subset S$ of $L(G)$ is called the Laplacian ideal of $G$. If $I \subset S$ is the Laplacian ideal of $G$, the lattice ideal $I(\mathcal{L})=\left(I:\left(t_{1} \cdots t_{s}\right)^{\infty}\right)$ is called the toppling ideal of $G[23,32]$. If $G$ is connected, the toppling ideal has dimension 1.

The torsion subgroup of the factor group $\mathbb{Z}^{s} / \operatorname{Im}(L(G))$, denoted by $K(G)$, is called the critical group or the sandpile group of $G[1,22]$. The group $K(G)$ is equal to the torsion subgroup of $\mathbb{Z}^{s} / \mathcal{L}$. Below, we denote the set of edges of $G$ incident to $t_{i}$ by $E\left(t_{i}\right)$. Thus we can give an application of our earlier results to this setting.

Proposition 7.1. Let $G=(V, E, w)$ be a connected weighted simple graph with vertices $t_{1}, \ldots, t_{s}$ and let $I \subset S$ be its Laplacian ideal. Then the following hold.

(a) $V\left(I, t_{i}\right)=\{0\}$ for all $i$.

(b) $\operatorname{deg}(S / I)=\operatorname{deg}(S / I(\mathcal{L}))=|K(G)|$.

(c) $\operatorname{Hull}(I)=I(\mathcal{L})$.

(d) If $\left|E\left(t_{i}\right)\right| \geq 3$ for all $i$, then $I$ is not a lattice ideal.

(e) If $G=\mathcal{K}_{s}$ is a complete graph, then $\operatorname{deg}(S / I)=s^{s-2}$.

As another application, we show that the Laplacian ideal is an almost complete intersection for any connected simple graph without vertices of degree 1 (Proposition 7.3).

Given a square integer matrix $L$, we denote its underlying digraph by $G_{L}$ (Definition 7.4). If $L$ is a GCB matrix and $G_{L}$ is strongly connected, we show that $L^{\top}$ is a GCB matrix (Theorem 7.5). If $L$ is a GCB matrix, we show that $G_{L}$ is strongly connected if and only if $V\left(I(L), t_{i}\right)=\{0\}$ for all $i$ (Proposition 7.6). Thus, the results of the previous sections can also be applied to GCB ideals that arise from matrices with strongly connected underlying digraphs.

Finally, in section 8 we focus on matrix ideals with $s=3$ and apply our earlier results in this setting. From [31, Theorem 6.1], it follows that graded lattice ideals of height 2 in 3 variables are generated by at most 3 binomials. The main results of section 8 uncover the structure of this type of ideal and the structure of graded lattices of rank 2 in $\mathbb{Z}^{3}$. We show that a graded lattice ideal in $K\left[t_{1}, t_{2}, t_{3}\right]$ of height 2 is generated by a full set of critical binomials (Definition 8.1, Theorem 8.2); our proof follows that of [19, pp. 137-140]. This result complements the well-known result of Herzog [16] showing that the toric ideal of a monomial space curve is generated by a full set of critical binomials.

It is easy to see that an ideal $I \subset K\left[t_{1}, t_{2}\right]$ is a graded lattice ideal of dimension 1 if and only if $I$ is a PCB ideal. The main result of section 8 is the analogue of this result in the case of 3 variables. Concretely, an ideal $I \subset K\left[t_{1}, t_{2}, t_{3}\right]$ is a graded lattice ideal of dimension 1 if and only if $I$ is a CB ideal (Theorem 8.6). Then we show that the graded lattices of rank 2 in $\mathbb{Z}^{3}$ are precisely the lattices generated by the columns of a CB matrix of size 3 (Corollary 8.7). As a corollary of Theorem 8.6, and for $s=3$, we deduce a characterization of the structure of the hull of a GCB ideal (Corollary 8.9).

For all unexplained terminology and additional information, we refer to $[10,27,36]$ (for the theory of binomial and lattice ideals), [9, 15] (for Gröbner bases and Hilbert functions), and [5, 25, 38] (for commutative algebra). 
2. Preliminaries. In this section, we present some of the results that will be needed throughout the paper and introduce some more notation. All results of this section are well known. To avoid repetition, we continue to employ the notation and definitions used in section 1.

Let $S=K\left[t_{1}, \ldots, t_{s}\right]$ be a polynomial ring over a field $K$ and let $I$ be an ideal of $S$. As usual, $\mathfrak{m}$ will denote the maximal ideal of $S$ generated by $t_{1}, \ldots, t_{s}$. The vector space of polynomials in $S$ (resp., $I$ ) of degree at most $i$ is denoted by $S_{\leq i}$ (resp., $I_{\leq i}$ ). The functions

$$
H_{I}^{a}(i)=\operatorname{dim}_{K}\left(S_{\leq i} / I_{\leq i}\right) \quad \text { and } \quad H_{I}(i)=H_{I}^{a}(i)-H_{I}^{a}(i-1)
$$

are called the affine Hilbert function and the Hilbert function of $S / I$, respectively.

Let $k=\operatorname{dim}(S / I)$ be the Krull dimension of $S / I$. According to [15, Remark 5.3.16, p. 330], there are unique polynomials $h_{I}^{a}(t)=\sum_{j=0}^{k} a_{j} t^{j}$ and $h_{I}(t)=\sum_{j=0}^{k-1} c_{j} t^{j}$ in $\mathbb{Q}[t]$ of degrees $k$ and $k-1$, respectively, such that $h_{I}^{a}(i)=H_{I}^{a}(i)$ and $h_{I}(i)=H_{I}(i)$ for $i \gg 0$. By convention, the zero polynomial has degree -1 . Notice that $a_{k}(k !)=$ $c_{k-1}\left((k-1)\right.$ !) for $k \geq 1$. If $k=0$, then $H_{I}^{a}(i)=\operatorname{dim}_{K}(S / I)$ for $i \gg 0$.

Definition 2.1. The integer $a_{k}(k !)$, denoted by $\operatorname{deg}(S / I)$, is called the degree of $S / I$.

Remark 2.2. If $S=\oplus_{i=0}^{\infty} S_{i}$ has the standard grading and $I \subset S$ is a graded ideal, then $H_{I}(i)$ is equal to $\operatorname{dim}_{K}\left(S_{i} / I_{i}\right)$ for all $i$, and $H_{I}^{a}$ is the Hilbert-Samuel function of $S / I$ with respect to $\mathfrak{m}$ in the sense of [35, Definition B.3.1].

We will use the following multi-index notation: for $a=\left(a_{1}, \ldots, a_{s}\right) \in \mathbb{Z}^{s}$, set $t^{a}=t_{1}^{a_{1}} \cdots t_{s}^{a_{s}}$. Notice that $t^{a}$ is a monomial in the Laurent polynomial ring $T:=$ $K\left[t_{1}^{ \pm 1}, \ldots, t_{s}^{ \pm 1}\right]$. If $a_{i} \geq 0$ for all $i, t^{a}$ is just a monomial in $S$.

DEFINITION 2.3. The graded reverse lexicographical order (GRevLex for short) on the monomials of $S$ is defined as $t^{b} \succ t^{a}$ if and only if $\operatorname{deg}\left(t^{b}\right)>\operatorname{deg}\left(t^{a}\right)$, or $\operatorname{deg}\left(t^{b}\right)=\operatorname{deg}\left(t^{a}\right)$ and the last nonzero entry of $b-a$ is negative.

Let $\succ$ be a monomial order on $S$ and let $I \subset S$ be an ideal. As usual, if $g$ is a polynomial of $S$, we will denote the leading term of $g$ by in $(g)$ and the initial ideal of $I$ by in $(I)$. We refer to [9] for the theory of Gröbner bases. Let $u=t_{s+1}$ be a new variable. For $f \in S$ of degree $e$ define

$$
f^{h}=u^{e} f\left(t_{1} / u, \ldots, t_{s} / u\right) ;
$$

that is, $f^{h}$ is the homogenization of the polynomial $f$ with respect to $u$. The homogenization of $I$ is the ideal $I^{h}$ of $S[u]$ given by $I^{h}=\left(f^{h} \mid f \in I\right)$, and $S[u]$ is given the standard grading.

The Gröbner bases of $I$ and $I^{h}$ are nicely related.

LEMMA 2.4. Let I be an ideal of $S$ and let $\succ$ be the GRevLex order on $S$ and $S[u]$, respectively.

(a) If $g_{1}, \ldots, g_{r}$ is a Gröbner basis of $I$, then $g_{1}^{h}, \ldots, g_{r}^{h}$ is a Gröbner basis of $I^{h}$.

(b) $H_{I}^{a}(i)=H_{I^{h}}(i)$ for $i \geq 0$.

(c) $\operatorname{deg}(S / I)=\operatorname{deg}\left(S[u] / I^{h}\right)$.

Proof. (a) This follows readily from [36, Propositions 2.4.26 and 2.4.30]. (b) Fix $i \geq 0$. The mapping $S[u]_{i} \rightarrow S_{\leq i}$ induced by mapping $u \mapsto 1$ is a $K$-linear surjection. Consider the induced composite $K$-linear surjection $S[u]_{i} \rightarrow S_{\leq i} \rightarrow S_{\leq i} / I_{\leq i}$. An easy check shows that this has kernel $I_{i}^{h}$. Hence, we have a $K$-linear isomorphism of finite-dimensional $K$-vector spaces

$$
S[u]_{i} / I_{i}^{h} \simeq S_{\leq i} / I_{\leq i} .
$$

Copyright (c) by SIAM. Unauthorized reproduction of this article is prohibited. 
Thus $H_{I}^{a}(i)=H_{I^{h}}(i)$. (c) From classical theory [38, p. 192], $\operatorname{dim}\left(S[u] / I^{h}\right)$ is equal to $\operatorname{dim}(S / I)+1$. Hence the equality follows from (b).

Proposition 2.5. Let $I$ be an ideal of $S$ and let $\mathfrak{p}_{1}, \ldots, \mathfrak{p}_{r}$ be the set of associated primes of I of dimension $\operatorname{dim}(S / I)$. If $I=\mathfrak{q}_{1} \cap \cdots \cap \mathfrak{q}_{m}$ is a minimal primary decomposition of $I$ such that $\operatorname{rad}\left(\mathfrak{q}_{i}\right)=\mathfrak{p}_{i}$ for $i=1, \ldots, r$, then $\operatorname{deg}(S / I)=\sum_{i=1}^{r} \operatorname{deg}\left(S / \mathfrak{q}_{i}\right)$.

Proof. By [38, p. 181, Theorem 17, items [7]-[9]] and [38, p. 192], $\mathfrak{q}_{1}^{h}, \ldots, \mathfrak{q}_{r}^{h}$ are the primary components of $I^{h}$ of maximal dimension. Hence, by (part of) the associativity law of multiplicities (cf. [15, Lemma 5.3.11, p. 327]), we get

$$
\operatorname{deg}\left(S[u] / I^{h}\right)=\sum_{i=1}^{r} \operatorname{deg}\left(S[u] / \mathfrak{q}_{i}^{h}\right) .
$$

Hence, by Lemma 2.4(c), $\operatorname{deg}(S / I)=\sum_{i=1}^{r} \operatorname{deg}\left(S / \mathfrak{q}_{i}\right)$.

DEFINITION 2.6. The torsion subgroup of an abelian group $(M,+)$, denoted by $T(M)$, is the set of all $x$ in $M$ such that $\ell x=0$ for some $\ell \in \mathbb{N}_{+}$.

Binomial and lattice ideals. Let $\mathcal{L} \subset \mathbb{Z}^{s}$ be a lattice and let $I(\mathcal{L}) \subset S$ be its lattice ideal. It is well known that the height of $I(\mathcal{L})$ is the rank of $\mathcal{L}$ and that $I(\mathcal{L})$ is a toric ideal if and only if $\mathbb{Z}^{s} / \mathcal{L}$ is free as a $\mathbb{Z}$-module [27, p. 131]. Let $p$ be the characteristic of the field $K$. The next result will be relevant in our context because it says that when $p=0$, or when $p>0$ and $p$ is relatively prime to the cardinality of the torsion subgroup of $\mathbb{Z}^{s} / \mathcal{L}$, then $I(\mathcal{L})$ is a radical ideal and hence $I(\mathcal{L})$ has a unique minimal primary decomposition (see Theorem 3.3).

TheOREm 2.7 (see [13, pp. 99-106]). If $p=0$, then $\operatorname{rad}(I(\mathcal{L}))=I(\mathcal{L})$, and if $p>0$, then

$$
\operatorname{rad}(I(\mathcal{L}))=\left(t^{a}-t^{b} \mid p^{r}(a-b) \in \mathcal{L} \text { for some } r \in \mathbb{N}\right) .
$$

The degree is independent of the base field $K$.

Proposition 2.8. If $I_{\mathbb{Q}}(\mathcal{L})$ is the lattice ideal of $\mathcal{L}$ over the field $\mathbb{Q}$, then

$$
\operatorname{deg}(S / I(\mathcal{L}))=\operatorname{deg}\left(\mathbb{Q}\left[t_{1}, \ldots, t_{s}\right] / I_{\mathbb{Q}}(\mathcal{L})\right) .
$$

Proof. Let $\succ$ be the GRevLex order on $S$ and $S_{\mathbb{Q}}=\mathbb{Q}\left[t_{1}, \ldots, t_{s}\right]$, and on the extensions $S[u]$ and $S_{\mathbb{Q}}[u]$, respectively. Let $G_{\mathbb{Q}}$ be the reduced Gröbner basis of $I_{\mathbb{Q}}(\mathcal{L})$. We set $I=I(\mathcal{L})$ and $I_{\mathbb{Q}}=I_{\mathbb{Q}}(\mathcal{L})$. Notice that $\mathbb{Z} / p \mathbb{Z} \subset K$, where $p=\operatorname{char}(K)$. Hence $S_{\mathbb{Z}}=\mathbb{Z}\left[t_{1}, \ldots, t_{s}\right]$ maps into $S$. If $G$ denotes the image of $G_{\mathbb{Q}}$ under this map, then using Buchberger's criterion [9, p. 84], it is seen that $G$ is a Gröbner basis of $I$. Hence, by Lemma 2.4(a), $G_{\mathbb{Q}}^{h}$ and $G^{h}$ are Gröbner bases of $I_{\mathbb{Q}}^{h}$ and $I^{h}$, respectively, where $G^{h}$ is the set of all $f^{h}$ with $f \in G$. Therefore, the rings $S_{\mathbb{Q}}[u] / I_{\mathbb{Q}}^{h}$ and $S[u] / I^{h}$ have the same Hilbert function. Thus, by Lemma 2.4, the result follows.

If $I \subset S$ is an ideal and $h \in S$, we set $(I: h):=\{f \in S \mid f h \in I\}$. This is the colon ideal of $I$ relative to $h$. The saturation of $I$ with respect to $h$ is the ideal $\left(I: h^{\infty}\right):=\cup_{k=1}^{\infty}\left(I: h^{k}\right)$.

The following is a well-known result that follows from [10, Corollary 2.5].

Theorem 2.9 (see [10]). Let I be a binomial ideal of $S$. Then the following are equivalent:

(a) I is a lattice ideal;

(b) $I=\left(I:\left(t_{1} \cdots t_{s}\right)^{\infty}\right)$;

(c) $t_{i}$ is a nonzero-divisor of $S / I$ for all $i$.

Lemma 2.10 (see [20]). Let $\mathcal{L} \subset \mathbb{Z}^{s}$ be a lattice. Then $\mathcal{L}$ is generated by $a_{1}, \ldots, a_{m}$ if and only if

$$
\left(\left(t^{a_{1}^{+}}-t^{a_{1}^{-}}, \ldots, t^{a_{m}^{+}}-t^{a_{m}^{-}}\right):\left(t_{1} \cdots t_{s}\right)^{\infty}\right)=I(\mathcal{L})
$$


If $B$ is a subset of $\mathbb{Z}^{s},\langle B\rangle$ will denote the subgroup of $\mathbb{Z}^{s}$ generated by $B$. Let $T$ be the Laurent polynomial ring $K\left[\mathbf{t}^{ \pm 1}\right]=K\left[t_{1}^{ \pm 1}, \ldots, t_{s}^{ \pm 1}\right]$. As usual, if $I$ is an ideal of $S, I T$ will denote its extension in $T$. Part (b) of the next result can be applied to any matrix ideal.

COROLlary 2.11.

(a) If $I(\mathcal{L})=\left(\left\{t^{a_{i}^{+}}-t^{a_{i}^{-}}\right\}_{i=1}^{m}\right)$, then $\mathcal{L}=\left\langle\left\{a_{i}\right\}_{i=1}^{m}\right\rangle$.

(b) If $I=\left(\left\{t^{a_{i}^{+}}-t^{a_{i}^{-}}\right\}_{i=1}^{m}\right) \subset S$ and $\mathcal{L}=\left\langle\left\{a_{i}\right\}_{i=1}^{m}\right\rangle$, then $I(\mathcal{L})=I T \cap S$.

Proof. (a) By Theorem 2.9, $\left(I(\mathcal{L}):\left(t_{1} \cdots t_{s}\right)^{\infty}\right)=I(\mathcal{L})$. Hence, by Lemma 2.10, $\mathcal{L}=\left\langle\left\{a_{i}\right\}_{i=1}^{m}\right\rangle$.

(b) See [10, Corollary 2.5] and its proof.

Normal forms and critical groups. Let $\mathcal{L} \subset \mathbb{Z}^{s}$ be a lattice of rank $r$ generated by $a_{1}, \ldots, a_{m}$ and let $L$ be the $s \times m$ matrix of rank $r$ with column vectors $a_{1}, \ldots, a_{m}$.

There are invertible integer matrices $P$ and $Q$ such that $P L Q=\Gamma$, where $\Gamma$ is an $s \times m$ "diagonal" matrix $\Gamma=\operatorname{diag}\left(\gamma_{1}, \gamma_{2}, \ldots, \gamma_{r}, 0, \ldots, 0\right)$ with $\gamma_{i} \in \mathbb{N}_{+}$and $\gamma_{i} \mid \gamma_{j}$ if $i \leq j$. The matrix $\Gamma$ is called the normal form of $L$ and the expression $P L Q=\Gamma$ the normal decomposition of $L$ ( $\Gamma$ is also called the Smith normal form of $L$ ). The integers $\gamma_{1}, \ldots, \gamma_{r}$ are the invariant factors of $L$. The greatest common divisor of all the nonzero $i \times i$ subdeterminants of $L$ will be denoted by $\Delta_{i}(L)$. The cardinality of a finite set $C$ is denoted by $|C|$.

Definition 2.12. The group $T\left(\mathbb{Z}^{s} / \mathcal{L}\right)$ is called the critical group of $\mathcal{L}$ or the critical group of $L$.

Critical groups of lattices will play an important role in several parts of the paper. Their structure can easily be determined using the next result, which follows from the fundamental structure theorem for finitely generated abelian groups [17]. Thus, using any algebraic system that computes normal forms of matrices, Maple [7] for instance, one can determine the structure of critical groups.

THEOREM 2.13.

(a) [17, Theorem 3.9] $\gamma_{1}=\Delta_{1}(L), \gamma_{i}=\Delta_{i}(L) \Delta_{i-1}(L)^{-1}$ for $i=2, \ldots, r$.

(b) $\left[17\right.$, pp. 187-188] $\mathbb{Z}^{s} / \mathcal{L} \simeq \mathbb{Z} /\left(\gamma_{1}\right) \oplus \mathbb{Z} /\left(\gamma_{2}\right) \oplus \cdots \oplus \mathbb{Z} /\left(\gamma_{r}\right) \oplus \mathbb{Z}^{s-r}$.

(c) $T\left(\mathbb{Z}^{s} / \mathcal{L}\right) \simeq \mathbb{Z} /\left(\gamma_{1}\right) \oplus \mathbb{Z} /\left(\gamma_{2}\right) \oplus \cdots \oplus \mathbb{Z} /\left(\gamma_{r}\right)$.

(d) $\Delta_{r}(L)=\left|T\left(\mathbb{Z}^{s} / \mathcal{L}\right)\right|=\gamma_{1} \cdots \gamma_{r}$.

(e) $\left|T\left(\mathbb{Z}^{s} / \mathcal{L}\right)\right|=\left|T\left(\mathbb{Z}^{m} / \mathcal{L}^{\top}\right)\right|$, where $\mathcal{L}^{\top}$ is the lattice of $\mathbb{Z}^{m}$ spanned by the rows of $L$.

If $m=s$, let $h_{i, j}$ be the $(i, j)$-minor of $L$, i.e., the determinant of the matrix obtained from $L$ by eliminating the $i$ th row and the $j$ th column of $L$. Let $L_{i, j}=$ $(-1)^{i+j} h_{j, i}$ and set $\operatorname{adj}(L)=\left(L_{i, j}\right)$, the adjoint matrix of $L$. Note that $L_{i, i}=h_{i, i}$. The adjoint matrix will come up later in Theorem 6.3 and Proposition 7.6.

3. Degree and torsion in primary decompositions. In this section, we study primary decompositions of lattice ideals over an arbitrary field, using the Eisenbud-Sturmfels theory of binomial ideals over algebraically closed fields [10]. For a graded lattice ideal of dimension 1, we give the explicit minimal primary decomposition over a field with enough roots of unity.

Lemma 3.1. Let $F$ be a field extension of $K$, let $B=F\left[t_{1}, \ldots, t_{s}\right]$ be a polynomial ring with coefficients in $F$, and let $I$ be an ideal of $S$. Then the following hold.

(a) $I B \cap S=I$.

(b) $\operatorname{deg}(S / I)=\operatorname{deg}(B / I B)$.

(c) If $\mathfrak{q}$ is a $\mathfrak{p}$-primary ideal of $B$, then $\mathfrak{q} \cap S$ is a $\mathfrak{p} \cap S$-primary ideal of $S$.

(d) If $I B=\cap_{i=1}^{r} \mathfrak{q}_{i}$ is a primary decomposition of $I B$, then $I=\cap_{i=1}^{r}\left(\mathfrak{q}_{i} \cap S\right)$ is a primary decomposition of $I$ such that $\operatorname{rad}\left(\mathfrak{q}_{i} \cap S\right)=\operatorname{rad}\left(\mathfrak{q}_{i}\right) \cap S$. 
(e) If $I$ is the lattice ideal of $\mathcal{L}$ in $S$, then $I B$ is the lattice ideal of $\mathcal{L}$ in $B$.

Proof. Let $G=\left\{g_{1}, \ldots, g_{m}\right\}$ be a Gröbner basis of $I$ with respect to the GRevLex order. By Buchberger's criterion [9, Theorem 6, p. 84], $G$ is also a Gröbner basis for $I B$ with respect to the GRevLex order on $B$.

(a) Note that $K \hookrightarrow F$ is a faithfully flat extension. Apply the functor $(-) \otimes_{K} S$. By base change, it follows that $S \hookrightarrow B$ is a faithfully flat extension. Hence, by [25, Theorem 7.5(ii)], $I=I B \cap S$ for any ideal $I$ of $S$.

(b) By Lemma 2.4(a), $I^{h}$ and $I B^{h}$ are both generated by $G^{h}=\left\{g_{1}^{h}, \ldots, g_{m}^{h}\right\}$, and $G^{h}$ is a Gröbner basis for $I B^{h}$. Hence, by Lemma 2.4(b), we get

$H_{I}^{a}(i)=H_{I^{h}}(i)=\operatorname{dim}_{K}\left(S[u] / I^{h}\right)_{i}=\operatorname{dim}_{F}\left(B[u] / I B^{h}\right)_{i}=H_{I B^{h}}(i)=H_{I B}^{a}(i)$

for $i \geq 0$. Therefore, one has the equality $\operatorname{deg}(S / I)=\operatorname{deg}(B / I B)$.

(c) This is well known and not hard to show.

(d) This follows from (a) and (c).

(e) Let $t^{a_{1}^{+}}-t^{a_{1}^{-}}, \ldots, t^{a_{m}^{+}}-t^{a_{m}^{-}}$be a set of generators of $I$. This set also generates $I B$. Then, by Lemma 2.10 , one has

$$
\left(I B:_{B}\left(t_{1} \cdots t_{s}\right)^{\infty}\right)=I_{B}
$$

where $I_{B}$ is the lattice ideal of $\mathcal{L}$ in $B$. We claim that $t_{i}$ is not a zero divisor of $B / I B$ for $i=1, \ldots, s$. Note that since $S \hookrightarrow B$ is a flat extension, applying the functor $(-) \otimes_{S} S / I$ and using base change, we deduce that $S / I \hookrightarrow B / I B$ is a flat extension. Hence since the map $S / I \stackrel{t_{i}}{\longrightarrow} S / I$ is injective, by Theorem 2.9, so is the map $B / I B \stackrel{t_{i}}{\longrightarrow} B / I B$. Consequently, by the claim, the left-hand side of (3.1) is equal to $I B$ and we get the equality $I B=I_{B}$.

We come to the first main result of this section.

THEOREM 3.2. Let $I(\mathcal{L})$ be a lattice ideal of $S$ over an arbitrary field $K$ of characteristic $p$, let $c$ be the number of associated primes of $I(\mathcal{L})$, and for $p>0$, let $G$ be the unique largest subgroup of $T\left(\mathbb{Z}^{s} / \mathcal{L}\right)$ whose order is relatively prime to $p$. Then

(a) all associated primes of $I(\mathcal{L})$ have height equal to $\operatorname{rank}(\mathcal{L})$;

(b) $\left|T\left(\mathbb{Z}^{s} / \mathcal{L}\right)\right| \geq c$ if $p=0$ and $|G| \geq c$ if $p>0$ with equality if $K$ is algebraically closed;

(c) $\operatorname{deg}(S / I(\mathcal{L})) \geq\left|T\left(\mathbb{Z}^{s} / \mathcal{L}\right)\right|$ if $p=0$ and $\operatorname{deg}(S / I(\mathcal{L})) \geq|G|$ if $p>0$.

Proof. Let $\bar{K}$ be the algebraic closure of $K$ and let $\bar{S}=\bar{K}\left[t_{1}, \ldots, t_{s}\right]$ be the corresponding polynomial ring with coefficients in $\bar{K}$. Thus, we have an integral extension $S \subset \bar{S}$ of normal domains. We set $I=I(\mathcal{L})$ and $\bar{I}=I \bar{S}$, where the latter is the extension of $I$ to $\bar{S}$. The ideal $\bar{I}$ is the lattice ideal of $\mathcal{L}$ in $\bar{S}$ (see Lemma 3.1(e)). Hence, as $\bar{K}$ is algebraically closed, by [10, Corollaries 2.2 and 2.5] $\bar{I}$ has a unique minimal primary decomposition

$$
\bar{I}=\overline{\mathfrak{q}}_{1} \cap \cdots \cap \overline{\mathfrak{q}}_{c_{1}},
$$

where $c_{1}=\left|T\left(\mathbb{Z}^{s} / \mathcal{L}\right)\right|$ if $p=0$ and $c_{1}=|G|$ if $p>0$. Notice that $c_{1}=\left|T\left(\mathbb{Z}^{s} / \mathcal{L}\right)\right|$ if $p$ is relatively prime to $\left|T\left(\mathbb{Z}^{s} / \mathcal{L}\right)\right|$. Furthermore, also by [10, Corollaries 2.2 and 2.5], one has that if $\overline{\mathfrak{p}}_{i}=\operatorname{rad}\left(\overline{\mathfrak{q}}_{i}\right)$ for $i=1, \ldots, c_{1}$, then $\overline{\mathfrak{p}}_{1}, \ldots, \overline{\mathfrak{p}}_{c_{1}}$ are the associated primes of $\bar{I}$ and $\operatorname{ht}\left(\overline{\mathfrak{p}}_{i}\right)=\operatorname{rank}(\mathcal{L})$ for $i=1, \ldots, c_{1}$. Hence, by Lemma 3.1(d), one has a primary decomposition

$$
I=\left(\overline{\mathfrak{q}}_{1} \cap S\right) \cap \cdots \cap\left(\overline{\mathfrak{q}}_{c_{1}} \cap S\right)
$$

such that $\operatorname{rad}\left(\overline{\mathfrak{q}}_{i} \cap S\right)=\operatorname{rad}\left(\overline{\mathfrak{q}}_{i}\right) \cap S=\overline{\mathfrak{p}}_{i} \cap S$. We set $\mathfrak{p}_{i}=\overline{\mathfrak{p}}_{i} \cap S$ and $\mathfrak{q}_{i}=\overline{\mathfrak{q}}_{i} \cap S$ for $i=1, \ldots, c_{1}$. 
(a) Since $S$ is a normal domain and $S \subset \bar{S}$ is an integral extension, we get $\operatorname{ht}\left(\mathfrak{p}_{i}\right)=\operatorname{ht}\left(\overline{\mathfrak{p}}_{i}\right)=\operatorname{rank}(\mathcal{L})$ for all $i$ (see [24, Theorems 5 and 20]).

(b) By (3.3), the associated primes of $I$ are contained in $\left\{\mathfrak{p}_{1}, \ldots, \mathfrak{p}_{c_{1}}\right\}$. Thus, $c_{1} \geq c$, which proves the first part. Now, assume that $K=\bar{K}$. By (a), we may assume that $\mathfrak{p}_{1}, \ldots, \mathfrak{p}_{c}$ are the minimal primes of $I$. Consequently, $I$ has a unique minimal primary decomposition $I=\mathfrak{Q}_{1} \cap \cdots \cap \mathfrak{Q}_{c}$ such that $\mathfrak{Q}_{i}$ is $\mathfrak{p}_{i}$-primary and $\operatorname{ht}\left(\mathfrak{p}_{i}\right)=\operatorname{rank}(\mathcal{L})$ for $i=1, \ldots, c$. As $I=\bar{I}$, from (3.2), we get that $c_{1}=c$.

(c) Using Lemma 3.1(b), (3.2) that was stated at the beginning of the proof, and the additivity of the degree (Proposition 2.5), we get

$$
\operatorname{deg}(S / I)=\operatorname{deg}(\bar{S} / \bar{I})=\sum_{i=1}^{c_{1}} \operatorname{deg}\left(\bar{S} / \overline{\mathfrak{q}}_{i}\right) \geq c_{1} .
$$

Primary decompositions of graded lattice ideals. The aim here is to give explicitly the minimal primary decomposition of a graded lattice ideal of dimension 1 in terms of the normal decomposition of an integer matrix (see section 2).

Let $\mathcal{L}$ be a lattice in $\mathbb{Z}^{s}$ of rank $s-1$ generated by $a_{1}, \ldots, a_{m}$ and let $L$ be the $s \times m$ matrix with column vectors $a_{1}, \ldots, a_{m}$. There are invertible integer matrices $P=\left(p_{i j}\right)$ and $Q$ such that $P L Q=\Gamma$, where $\Gamma=\operatorname{diag}\left(\gamma_{1}, \gamma_{2}, \ldots, \gamma_{s-1}, 0, \ldots, 0\right)$ with $\gamma_{i} \in \mathbb{N}_{+}$and $\gamma_{i} \mid \gamma_{j}$ if $i \leq j$. The torsion subgroup of $\mathbb{Z}^{s} / \mathcal{L}$ has order $\gamma:=\gamma_{1} \cdots \gamma_{s-1}$. If $p_{i, *}$ denotes the $i$ th row of $P$, then the last row of $P$ satisfies $\operatorname{gcd}\left(p_{s, *}\right)=1$, $p_{s, *} L=0$, and $\mathcal{L} \subset \operatorname{ker}\left(p_{s, *}\right)$. This follows using the technique described in [28, p. 37] to solve systems of linear equations over the integers. Thus $\operatorname{ker}\left(p_{s, *}\right)$ is equal to $\mathcal{L}_{s}$, the saturation of $\mathcal{L}$ in the sense of [10]. For convenience recall that $\mathcal{L}_{s}$ is the set of all $a \in \mathbb{Z}^{s}$ such that $\eta a \in \mathcal{L}$ for some $0 \neq \eta \in \mathbb{Z}$.

For the rest of this section we suppose that $K$ is a field containing the $\gamma_{s-1}$ th roots of unity with $\operatorname{char}(K)=0$ or $\operatorname{char}(K)=p, p$ a prime with $p \nmid \gamma_{s-1}$. Under this assumption, the lattice ideal $I(\mathcal{L})$ is radical because $\operatorname{char}(K)=0$ or $\operatorname{gcd}\left(p, \mid T\left(\mathbb{Z}^{s} / \mathcal{L} \mid\right)=1\right.$ (see Theorem 2.7), and for each $i$ the polynomial $z^{\gamma_{i}}-1$ has $\gamma_{i}$ distinct roots in $K$. Write $\Lambda_{i}$ to denote the set of $\gamma_{i}$ th roots of unity in $K$ and $\Lambda=\prod_{i=1}^{s-1} \Lambda_{i}$. The set $\Lambda$ is a group under componentwise multiplication and $|\Lambda|=\gamma$. We also suppose that $I(\mathcal{L})$ is graded with respect to a weight vector $\mathbf{d}=\left(d_{1}, \ldots, d_{s}\right)$ in $\mathbb{N}_{+}^{s}$ with $\operatorname{gcd}(\mathbf{d})=1$. Notice that $\mathbf{d}= \pm p_{s, *}$. This means that in the nongraded case, $\pm p_{s, *}$ can play the role of $\mathbf{d}$. Consider a polynomial ring $K\left[x_{1}\right]$ in one variable. For any $\lambda=\left(\lambda_{1}, \ldots, \lambda_{s-1}\right) \in \Lambda$, there is an homomorphism of $K$-algebras

$$
\varphi_{\lambda}: S \rightarrow K\left[x_{1}\right], \quad t_{i} \longmapsto \lambda_{1}^{p_{1, i}} \cdots \lambda_{s-1}^{p_{s-1, i}} x_{1}^{d_{i}}, \quad i=1, \ldots, s .
$$

The kernel of $\varphi_{\lambda}$, denoted by $\mathfrak{a}_{\lambda}$, is a prime ideal of $S$ of height $s-1$ [30]. This type of ideal was introduced in [30] to study the algebraic properties of PCB ideals.

The primary decomposition of an arbitrary lattice ideal over an algebraically closed field is given in [10, Corollary 2.5]. This decomposition will be used to prove Theorem 4.6(c). The next result shows an explicit primary decomposition for the class of ideals under consideration.

THEOREM 3.3. If $I(\mathcal{L})$ is a graded lattice ideal of dimension 1 , then $I(\mathcal{L})=$ $\cap_{\lambda \in \Lambda} \mathfrak{a}_{\lambda}$ is the minimal primary decomposition of $I(\mathcal{L})$ into exactly $\gamma$ primary components.

Proof. First of all, recall from Theorem 2.7 that $I(L)$ is a radical ideal. For any $\lambda=\left(\lambda_{1}, \ldots, \lambda_{s-1}\right)$ in $\Lambda$, let $\rho_{\lambda}: \mathcal{L}_{s} \rightarrow K^{*}$ be the partial character of $\mathcal{L}_{s}$ given by

$$
\rho_{\lambda}(\alpha)=\prod_{i=1}^{s-1} \lambda_{i}^{p_{i, 1} \alpha_{1}+\cdots+p_{i, s} \alpha_{s}}, \quad \text { where } \alpha=\left(\alpha_{1}, \ldots, \alpha_{s}\right) .
$$


Clearly $\rho_{\lambda}$ is a group homomorphism. First we show the following two conditions:

(i) $\rho_{\lambda}(\alpha)=1$ for $\alpha \in \mathcal{L}$; (ii) if $\rho_{\lambda}(\alpha)=1$ for all $\alpha \in \mathcal{L}_{s}$, then $\lambda=(1, \ldots, 1)$.

(i) Take $\alpha=\left(\alpha_{1}, \ldots, \alpha_{s}\right)$ in $\mathcal{L}$. To show $\rho_{\lambda}(\alpha)=1$ it suffices to prove

$$
p_{i, 1} \alpha_{1}+\cdots+p_{i, s} \alpha_{s} \equiv 0 \bmod \left(\gamma_{i}\right) \text { for all } i \text {. }
$$

As $\alpha$ is a linear combination of the columns of $L$, one has $\alpha^{\top}=L \mu^{\top}=$ $P^{-1} \Gamma Q^{-1} \mu^{\top}$ for some $\mu \in \mathbb{Z}^{m}$. Hence

$$
P \alpha^{\top}=\Gamma Q^{-1} \mu^{\top}=\left(\eta_{1} \gamma_{1}, \ldots, \eta_{s-1} \gamma_{s-1}, 0\right)^{\top}
$$

for some $\eta_{i}$ 's in $\mathbb{Z}$. Thus (3.4) holds, as required.

(ii) Fix $k$ such that $1 \leq k \leq s-1$. We set $P^{-1}=\left(r_{i j}\right)$ and denote by $r_{*, k}$ the $k$ th column of $P^{-1}$. Then $P^{-1} e_{k}^{\top}=r_{*, k}$ and $P r_{*, k}=e_{k}^{\top}$, where $e_{k}$ is the $k$ th unit vector. Since $P P^{-1}=I$, we get that $\left\langle p_{i, *}, r_{*, k}\right\rangle$ is 1 if $i=k$ and is 0 otherwise. In particular $\left\langle p_{s, *}, r_{*, k}\right\rangle=0$, i.e, $r_{*, k}$ is in $\mathcal{L}_{s}=\operatorname{ker}\left(p_{s, *}\right)$. Setting $\alpha^{\top}=r_{*, k}$ and using that $\rho_{\lambda}(\alpha)=1$, we get that $\lambda_{k}=1$.

Let $I_{\lambda}$ be the ideal of $S$ generated by all $t^{\alpha^{+}}-\rho_{\lambda}(\alpha) t^{\alpha^{-}}$with $\alpha \in \mathcal{L}_{s}$. Let us see that $I(\mathcal{L}) \subset I_{\lambda} \subset \mathfrak{a}_{\lambda}$ for all $\lambda \in \Lambda$. The first inclusion follows from (i). To show the second inclusion take $f=t^{\alpha^{+}}-\rho_{\lambda}(\alpha) t^{\alpha^{-}}$in $I_{\lambda}$ with $\alpha \in \mathcal{L}_{s}$. It is easy to see that $f \in \operatorname{ker}\left(\varphi_{\lambda}\right)$ using that $\alpha \in \operatorname{ker}(\mathbf{d})$ and the definition of $\rho_{\lambda}$. Hence, as $\mathfrak{a}_{\lambda}$ is a prime ideal of height $s-1$ for all $\lambda \in \Lambda$, we get that $\mathfrak{a}_{\lambda}$ is a primary component of $I(\mathcal{L})$ for all $\lambda \in \Lambda$. We claim that $\mathfrak{a}_{\lambda} \neq \mathfrak{a}_{v}$ if $\lambda \neq v$ and $\lambda, v \in \Lambda$. To show this assume that $\mathfrak{a}_{\lambda}=\mathfrak{a}_{v}$. Take an arbitrary $\alpha=\left(\alpha_{i}\right)$ in $\mathcal{L}_{s}$. Then $f=t^{\alpha^{+}}-\rho_{\lambda}(\alpha) t^{\alpha^{-}}$is in $I_{\lambda} \subset \mathfrak{a}_{\lambda}$. Thus $f \in \mathfrak{a}_{v}=\operatorname{ker}\left(\varphi_{v}\right)$. It follows readily that $\rho_{\lambda}(\alpha)=\rho_{v}(\alpha)$. Therefore $\rho_{\lambda v^{-1}}(\alpha)=1$ for all $\alpha \in \mathcal{L}_{s}$, and by (ii) $\lambda=v$. This proves the claim. Altogether $I(\mathcal{L})$ has at least $\gamma$ prime components and by Theorem 3.2(b) it has at most $\gamma$ prime components. Thus $I(\mathcal{L})=\cap_{\lambda \in \Lambda} \mathfrak{a}_{\lambda}$.

Remark 3.4. The ideal $I_{\lambda}$ is called the lattice ideal of $\mathcal{L}_{s}$ relative to the partial character $\rho_{\lambda}$ [10]. Since $\mathfrak{a}_{\lambda}$ is a prime ideal generated by "binomials" of the form $t^{\alpha^{+}}-\eta t^{\alpha^{-}}$, with $\eta \in K^{*}$, it follows that the inclusion $I_{\lambda} \subset \mathfrak{a}_{\lambda}$ is an equality.

4. The degree of lattice and toric ideals. In this section we show that the homogenization of a lattice ideal (resp., toric ideal) is again a lattice ideal (resp., toric ideal). For any toric or lattice ideal, we give formulas to compute the degree in terms of the torsion of certain factor groups of $\mathbb{Z}^{s}$ and in terms of relative volumes of lattice polytopes. A general reference for connections between monomial subrings, Ehrhart rings, polyhedra, and volume is [5, Chapters 5 and 6] (see also [12, 34, 37]).

Let $\mathcal{L} \subset \mathbb{Z}^{s}$ be a lattice and let $e_{i}$ be the $i$ th unit vector in $\mathbb{Z}^{s+1}$. For $a=\left(a_{i}\right) \in \mathbb{Z}^{s}$ define the value of $a$ as $|a|=\sum_{i=1}^{s} a_{i}$, and the homogenization of $a$ with respect to $e_{s+1}$ as $a^{h}=(a, 0)-|a| e_{s+1}$ if $|a| \geq 0$ and $a^{h}=(-a)^{h}$ if $|a|<0$. The choice of the last coordinate of $a^{h}$ is a convenience. The homogenization of $\mathcal{L}$, denoted by $\mathcal{L}^{h}$, is the lattice of $\mathbb{Z}^{s+1}$ generated by all $a^{h}$ such that $a \in \mathcal{L}$.

LEMma 4.1. Let $I(\mathcal{L}) \subset S$ be a lattice ideal and let $f_{1}, \ldots, f_{r}$ be a set of binomials such that the terms of $f_{i}$ have disjoint support for all $i$. Then the following hold.

(a) $I(\mathcal{L})^{h} \subset S[u]$ is a lattice ideal.

(b) If $\mathcal{L}=\left\langle b_{1}, \ldots, b_{r}\right\rangle$, then $\mathcal{L}^{h}=\left\langle b_{1}^{h}, \ldots, b_{r}^{h}\right\rangle$.

(c) $I\left(\mathcal{L}^{h}\right)=I(\mathcal{L})^{h}$.

(d) If $\left(\left(f_{1}, \ldots, f_{r}\right):\left(t_{1} \cdots t_{s}\right)^{\infty}\right)=I(\mathcal{L})$, then $\left(\left(f_{1}^{h}, \ldots, f_{r}^{h}\right):\left(t_{1} \cdots t_{s} u\right)^{\infty}\right)=I(\mathcal{L})^{h}$.

Proof. We set $I=I(\mathcal{L})$. Let $\succ$ denote the GRevLex order on $S$ and on $S[u]$, and let $g_{1}, \ldots, g_{m}$ be the reduced Gröbner basis of $I$. By Lemma $2.4(\mathrm{a}), g_{1}^{h}, \ldots, g_{m}^{h}$ is a Gröbner basis of $I^{h}$. 
(a) As $I^{h}$ is a binomial ideal, by Theorem 2.9 we need only show that $t_{i}$ is a nonzero divisor of $S[u] / I^{h}$ for $i=1, \ldots, s+1$, where $t_{s+1}=u$. First, we show the case $i=s+1$. Assume that $u f \in I^{h}$ for some $f \in S[u]$. By the division algorithm [11, Theorem 2.11], we can write

$$
f=h_{1} g_{1}^{h}+\cdots+h_{m} g_{m}^{h}+\mathfrak{f},
$$

where $h_{1}, \ldots, h_{m}, \mathfrak{f}$ are in $S[u]$ and $\mathfrak{f}$ is not divisible by any of the leading terms of $g_{1}^{h}, \ldots, g_{m}^{h}$. We claim that $\mathfrak{f}=0$. If $\mathfrak{f} \neq 0$, then $u \mathfrak{f} \in I^{h}$ and, consequently, $u \operatorname{in}(\mathfrak{f}) \in \operatorname{in}\left(I^{h}\right)$, where in $(\mathfrak{f})$ is the leading term of $\mathfrak{f}$ and in $\left(I^{h}\right)$ is the initial ideal of $I^{h}$. Hence, $u \operatorname{in}(\mathfrak{f})$ is a multiple of $\operatorname{in}\left(g_{\ell}^{h}\right)$ for some $\ell$. Since $u$ does not appear in the monomial $\operatorname{in}\left(g_{\ell}\right)=\operatorname{in}\left(g_{\ell}^{h}\right)$, we get that in(f) is divisible by $\operatorname{in}\left(g_{\ell}^{h}\right)$, a contradiction. Thus, $\mathfrak{f}=0$ and $f \in I^{h}$, as required. Next, we show the case $1 \leq i \leq s$. Assume that $t_{i} g \in I^{h}$ for some $i$ and some $g$ in $S[u]$. As $I^{h}$ is graded, we may assume that $g$ is homogeneous of degree $\delta$. Since $u$ is a nonzero divisor of $S[u] / I^{h}$, we may assume that $u$ does not divide the leading term of $g$. If $f=g\left(t_{1}, \ldots, t_{s}, 1\right)$, then $\operatorname{deg}(f)=\delta, f^{h}=g$, and $t_{i} f \in I$. Hence $f \in I$. Therefore, as $g_{1}, \ldots, g_{m}$ is a Gröbner basis, we can write $f=h_{1} g_{1}+\cdots+h_{m} g_{m}$, where $\operatorname{deg}(f) \geq \operatorname{deg}\left(h_{j} g_{j}\right)$ for all $j$. It is not hard to see that $g=f^{h} \in I^{h}$, as required.

(b) By changing the sign of $b_{i}$, if necessary, we may assume that $\left|b_{i}\right| \geq 0$ for all $i$. Clearly $\mathcal{L}^{h}$ contains $\left\langle b_{1}^{h}, \ldots, b_{r}^{h}\right\rangle$. Indeed, since $b_{i} \in \mathcal{L}$ for all $i$, we get $b_{i}^{h} \in \mathcal{L}^{h}$ for all $i$. Now, we prove that $\mathcal{L}^{h}$ is contained in $\left\langle b_{1}^{h}, \ldots, b_{r}^{h}\right\rangle$. It suffices to show that $c^{h} \in\left\langle b_{1}^{h}, \ldots, b_{r}^{h}\right\rangle$ for any $c \in \mathcal{L}$ with $|c| \geq 0$. By hypothesis, we can write $c=\lambda_{1} b_{1}+\cdots+\lambda_{r} b_{r}$ for some integers $\lambda_{1}, \ldots, \lambda_{r}$. Hence $|c|=\lambda_{1}\left|b_{1}\right|+\cdots+\lambda_{r}\left|b_{r}\right|$. From the last two equalities, we obtain that $c^{h}$ is equal to $\lambda_{1} b_{1}^{h}+\cdots+\lambda_{r} b_{r}^{h}$, as required.

(c) We can write $g_{i}=t^{a_{i}^{+}}-t^{a_{i}^{-}}$, with $\left|a_{i}\right| \geq 0$, for $i=1, \ldots, m$. By Corollary $2.11, \mathcal{L}$ is generated by $a_{1}, \ldots, a_{m}$. Then, by part $(\mathrm{b}), \mathcal{L}^{h}$ is generated by $a_{1}^{h}, \ldots, a_{m}^{h}$. Notice that $g_{i}^{h}$ is equal to $t^{\left(a_{i}^{h}\right)^{+}}-t^{\left(a_{i}^{h}\right)^{-}}$for all $i$. Therefore, using part (a) and Lemma 2.10, we get

$$
\begin{aligned}
I(\mathcal{L})^{h} & =\left(I(\mathcal{L})^{h}:\left(t_{1} \cdots t_{s} u\right)^{\infty}\right) \\
& =\left(\left(t^{\left(a_{1}^{h}\right)^{+}}-t^{\left(a_{1}^{h}\right)^{-}}, \ldots, t^{\left(a_{m}^{h}\right)^{+}}-t^{\left(a_{m}^{h}\right)^{-}}\right):\left(t_{1} \cdots t_{s} u\right)^{\infty}\right)=I\left(\mathcal{L}^{h}\right) .
\end{aligned}
$$

(d) This part follows from Lemma 2.10 and part (b).

Let $H=\left\{x^{v_{1}}, \ldots, x^{v_{s}}\right\} \subset K\left[x_{1}^{ \pm 1}, \ldots, x_{n}^{ \pm 1}\right]$ be a set of Laurent monomials, where $v_{i} \in \mathbb{Z}^{n}$, and let $K[H]$ be the $K$-subalgebra generated by $H$. There is an epimorphism of $K$-algebras

$$
\varphi: S=K\left[t_{1}, \ldots, t_{s}\right] \longrightarrow K[H], \quad t_{i} \longmapsto x^{v_{i}} .
$$

The kernel of $\varphi$, denoted by $P$, is called the toric ideal of $K[H]$. In general, $P$ is not a graded ideal. Since $S / P \simeq K[H]$, the degree of $K[H]$ is defined to be the degree of $S / P$.

Lemma 4.2. Let $P^{h} \subset S[u]$ be the homogenization of $P$. Then the following hold.

(a) $P^{h}$ is the toric ideal of $K\left[H^{\prime}\right]:=K\left[z, x^{v_{2}-v_{1}} z, \ldots, x^{v_{s}-v_{1}} z, x^{-v_{1}} z\right]$.

(b) The toric ideal of $K\left[x^{v_{1}} z, \ldots, x^{v_{s}} z, z\right]$ is the toric ideal $P^{h}$ of $K\left[H^{\prime}\right]$.

Proof. (a) The toric ideal of $K\left[H^{\prime}\right]$, denoted by $P^{\prime}$, is the kernel of the map $\varphi: S[u] \rightarrow K\left[H^{\prime}\right]$ induced by $t_{i} \mapsto x^{v_{i}-v_{1}} z$ for $i=1, \ldots, s+1$, where $v_{s+1}=0$ and 
$t_{s+1}=u$. Let $G$ be the reduced Gröbner basis of $P$ with respect to the GRevLex order. First, we show the inclusion $P^{h} \subset P^{\prime}$. Take an element $f$ of $G$. By Lemma 2.4(a), it suffices to show that $f^{h}$ is in $P^{\prime}$. We can write $f=t^{a^{+}}-t^{a^{-}}$with $\operatorname{in}(f)=t^{a^{+}}$. Thus, $\left|a^{+}\right| \geq\left|a^{-}\right|$and $f^{h}=t^{a^{+}}-t^{a^{-}} u^{|a|}$, where $a=a^{+}-a^{-}$. We set $a=\left(a_{1}, \ldots, a_{s}\right)$. From the equality

$$
0=a_{1} v_{1}+\cdots+a_{s} v_{s}=a_{2}\left(v_{2}-v_{1}\right)+\cdots+a_{s}\left(v_{s}-v_{1}\right)+\left(a_{1}+\cdots+a_{s}\right) v_{1},
$$

we get that $f^{h} \in P^{\prime}$, as required. Let $G^{\prime}$ be the reduced Gröbner basis of $P^{\prime}$ with respect to the GRevLex order. Next we show the inclusion $P^{\prime} \subset P^{h}$. Take an element $f^{\prime}$ of $G^{\prime}$. It suffices to show that $f^{\prime}$ is in $P^{h}$. As $f^{\prime}$ is homogeneous in the standard grading of $S[u]$, we can write $f^{\prime}=t^{c^{+}}-t^{c^{-}} u^{|c|}$ with in $\left(f^{\prime}\right)=t^{c^{+}}$and $c=\left(c_{1}, \ldots, c_{s}\right)$. Since $f^{\prime} \in P^{\prime}$, we get

$$
\left(z^{c_{1}^{+}}\right)\left(x^{v_{2}-v_{1}} z\right)^{c_{2}^{+}} \cdots\left(x^{v_{s}-v_{1}} z\right)^{c_{s}^{+}}=\left(z^{c_{1}^{-}}\right)\left(x^{v_{2}-v_{1}} z\right)^{c_{2}^{-}} \cdots\left(x^{v_{s}-v_{1}} z\right)^{c_{s}^{-}}\left(x^{-v_{1}} z\right)^{|c|} .
$$

Hence, $c_{2}\left(v_{2}-v_{1}\right)+\cdots+c_{s}\left(v_{s}-v_{1}\right)=-|c| v_{1}$. Consequently, $c_{1} v_{1}+\cdots+c_{s} v_{s}=0$, that is, the binomial $f=t^{c^{+}}-t^{c^{-}}$is in $P$. As $f^{\prime}=f^{h}$, we get $f^{\prime} \in P^{h}$.

(b) The map that sends $z$ to $x^{v_{1}} z$ induces an isomorphism $K\left[H^{\prime}\right] \rightarrow K\left[H^{\prime \prime}\right]$. So this part is an immediate consequence of (a)

Proposition 4.3 (see [12, Proposition 3.5], [37, Corollary 5.35]). Let $\mathcal{A}=$ $\left\{\alpha_{i}\right\}_{i=1}^{m}$ be a set of points of $\mathbb{Z}^{n}$ and let $\mathcal{P}=\operatorname{conv}(\mathcal{A})$ be the convex hull of $\mathcal{A}$. Then

$$
\left|T\left(\mathbb{Z}^{n} /\left(\alpha_{1}-\alpha_{m}, \ldots, \alpha_{m-1}-\alpha_{m}\right)\right)\right| \operatorname{deg}\left(K\left[x^{\alpha_{1}} z, \ldots, x^{\alpha_{m}} z\right]\right)=r ! \operatorname{vol}(\mathcal{P}),
$$

where $r=\operatorname{dim}(\mathcal{P}), \operatorname{vol}(\mathcal{P})$ is the relative volume of $\mathcal{P}$, and $z$ is a new variable.

Definition 4.4. The term $r \operatorname{vol}(\mathcal{P})$ is called the normalized volume of $\mathcal{P}$.

The next result holds for any toric ideal.

TheOREM 4.5. Let $P$ be the toric ideal of $K[H]=K\left[x^{v_{1}}, \ldots, x^{v_{s}}\right]$, let $A$ be the $n \times s$ matrix with column vectors $v_{1}, \ldots, v_{s}$, and let $r$ be the rank of $A$. Then

$$
\left|T\left(\mathbb{Z}^{n} /\left\langle v_{1}, \ldots, v_{s}\right\rangle\right)\right| \operatorname{deg}(S / P)=r ! \operatorname{vol}\left(\operatorname{conv}\left(v_{1}, \ldots, v_{s}, 0\right)\right) .
$$

Proof. By Lemma $2.4(\mathrm{c}), \operatorname{deg}(S / P)=\operatorname{deg}\left(S[u] / P^{h}\right)$. On the other hand, by Lemma $4.2(\mathrm{~b}), P^{h}$ is the toric ideal of the monomial subring

$$
K\left[H^{\prime \prime}\right]=K\left[x^{v_{1}} z, \ldots, x^{v_{s}} z, z\right] .
$$

Hence $S[u] / P^{h} \simeq K\left[H^{\prime \prime}\right]$. Therefore, setting $m=s+1, \alpha_{i}=v_{i}$ for $i=1, \ldots, m-1$, $\alpha_{m}=0$, and $\mathcal{A}=\left\{\alpha_{1}, \ldots, \alpha_{m}\right\}$, the result follows readily from Proposition 4.3.

We come to the main result of this section.

TheOREM 4.6. Let $\mathcal{L} \subset \mathbb{Z}^{s}$ be a lattice of rank $r$. Then the following hold.

(a) If $r=s$, then $\operatorname{deg}(S / I(\mathcal{L}))=\left|\mathbb{Z}^{s} / \mathcal{L}\right|$.

(b) If $r<s$, there is an integer matrix $A$ of size $(s-r) \times s$ with $\operatorname{rank}(A)=s-r$ such that we have the containment of rank $r$ lattices $\mathcal{L} \subset \operatorname{ker}_{\mathbb{Z}}(A)$ with equality if and only if $\mathbb{Z}^{s} / \mathcal{L}$ is torsion free.

(c) If $r<s$ and $v_{1}, \ldots, v_{s}$ are the columns of $A$, then

$$
\operatorname{deg}(S / I(\mathcal{L}))=\frac{\left|T\left(\mathbb{Z}^{s} / \mathcal{L}\right)\right|(s-r) ! \operatorname{vol}\left(\operatorname{conv}\left(0, v_{1}, \ldots, v_{s}\right)\right)}{\left|T\left(\mathbb{Z}^{s-r} /\left\langle v_{1}, \ldots, v_{s}\right\rangle\right)\right|} .
$$

Copyright $@$ by SIAM. Unauthorized reproduction of this article is prohibited. 
Proof. (a) By Lemma 2.4, $\operatorname{deg}(S / I(\mathcal{L}))=\operatorname{deg}\left(S[u] / I(\mathcal{L})^{h}\right)$, and by Lemma 4.1, $I(\mathcal{L})^{h}=I\left(\mathcal{L}^{h}\right)$. Since $S[u] / I\left(\mathcal{L}^{h}\right)$ has dimension 1, by [21, Theorem 3.12] the degree of $S[u] / I\left(\mathcal{L}^{h}\right)$ is $\left|T\left(\mathbb{Z}^{s+1} / \mathcal{L}^{h}\right)\right|$. Let $t^{a_{1}^{+}}-t^{a_{1}^{-}}, \ldots, t^{a_{m}^{+}}-t^{a_{m}^{-}}$be a set of generators of $I(\mathcal{L})$. By Corollary $2.11, \mathcal{L}$ is generated by $a_{1}, \ldots, a_{m}$. We may assume that $\left|a_{i}\right| \geq 0$ for all $i$. Then, by Lemma 4.1(b), $\mathcal{L}^{h}$ is generated by $a_{1}^{h}, \ldots, a_{m}^{h}$. Let $A$ and $A^{h}$ be the matrices with rows $a_{1}, \ldots, a_{m}$ and $a_{1}^{h}, \ldots, a_{m}^{h}$, respectively. Notice that $A^{h}$ is obtained from $A$ by adding the column vector $b=\left(-\left|a_{1}\right|, \ldots,-\left|a_{m}\right|\right)^{\top}$. Since $b$ is a linear combination of the columns of $A$, by the fundamental theorem of finitely generated abelian groups (see $[17$, p. 187] and Theorem 2.13), we get that the groups $\mathbb{Z}^{s} / \mathcal{L}$ and $\mathbb{Z}^{s+1} / \mathcal{L}^{h}$ have the same torsion. Thus, $\left|\mathbb{Z}^{s} / \mathcal{L}\right|$ is equal to $\left|T\left(\mathbb{Z}^{s+1} / \mathcal{L}^{h}\right)\right|$. Altogether, the degree of $S / I(\mathcal{L})$ is the order of $\mathbb{Z}^{s} / \mathcal{L}$.

(b) We may assume that $\mathcal{L}=\mathbb{Z} \alpha_{1} \oplus \cdots \oplus \mathbb{Z} \alpha_{r}$, where $\alpha_{1}, \ldots, \alpha_{s}$ is a $\mathbb{Q}$-basis of $\mathbb{Q}^{s}$. Consider the hyperplane $H_{i}$ of $\mathbb{Q}^{s}$ generated by $\alpha_{1}, \ldots, \widehat{\alpha}_{i}, \ldots, \alpha_{s}$. Note that the subspace of $\mathbb{Q}^{s}$ generated by $\alpha_{1}, \ldots, \alpha_{r}$ is equal to $H_{r+1} \cap \cdots \cap H_{s}$. There is a normal vector $w_{i} \in \mathbb{Z}^{s}$ such that

$$
H_{i}=\left\{\alpha \in \mathbb{Q}^{s} \mid\left\langle\alpha, w_{i}\right\rangle=0\right\} .
$$

It is not hard to see that the matrix $A$ with rows $w_{r+1}, \ldots, w_{s}$ is the matrix with the required conditions, because, by construction, $\alpha_{i} \in H_{j}$ for $i \neq j$ and, consequently, $w_{r+1}, \ldots, w_{s}$ are linearly independent. In particular we have the equality $\operatorname{rank}(\mathcal{L})=$ $\operatorname{rank}\left(\operatorname{ker}_{\mathbb{Z}}(A)\right)$.

(c) By Proposition 2.8 and Lemma 3.1(b), we may assume that $K$ is algebraically closed of characteristic zero. Let $P$ be the toric ideal of $K\left[x^{v_{1}}, \ldots, x^{v_{s}}\right]$ over the field $K$. By Theorem 4.5, we need only show the equality

$$
\operatorname{deg}(S / I(\mathcal{L}))=\left|T\left(\mathbb{Z}^{s} / \mathcal{L}\right)\right| \operatorname{deg}(S / P) .
$$

Let $\mathcal{L}_{s}=\left\{a \in \mathbb{Z}^{s} \mid \eta a \in \mathcal{L}\right.$ for some $\left.\eta \in \mathbb{Z} \backslash\{0\}\right\}$ be the saturation of $\mathcal{L}$. By part (b), $\mathcal{L}_{s}$ is equal to $\operatorname{ker}_{\mathbb{Z}}(A)$. We set $c=\left|T\left(\mathbb{Z}^{s} / \mathcal{L}\right)\right|$. Notice that $T\left(\mathbb{Z}^{s} / \mathcal{L}\right)=\mathcal{L}_{s} / \mathcal{L}$. Recall that a partial character of $\mathcal{L}_{s}$ is a homomorphism from the additive group $\mathcal{L}_{s}$ to the multiplicative group $K^{*}=K \backslash\{0\}$. According to [10, Corollaries 2.2 and 2.5], there exist distinct partial characters $\rho_{1}, \ldots, \rho_{c}$ of $\mathcal{L}_{s}$, extending the trivial character $\rho(a)=1$ for $a \in \mathcal{L}$, such that the minimal primary decomposition of $I(\mathcal{L})$ is given by

$$
I(\mathcal{L})=I_{\rho_{1}}\left(\mathcal{L}_{s}\right) \cap \cdots \cap I_{\rho_{c}}\left(\mathcal{L}_{s}\right),
$$

where $I_{\rho_{i}}\left(\mathcal{L}_{s}\right)$ is a prime ideal generated by all $t^{a^{+}}-\rho_{i}(a) t^{a^{-}}$with $a \in \mathcal{L}_{s}$. As $P$ is a minimal prime of $I(\mathcal{L})$ by part (b), we may assume $\rho_{1}(a)=1$ for $a \in \mathcal{L}_{s}$, i.e., $P=I_{\rho_{1}}\left(\mathcal{L}_{s}\right)$. By the additivity of the degree (see Proposition 2.5), we get

$$
\operatorname{deg}(S / I(\mathcal{L}))=\operatorname{deg}\left(S / I_{\rho_{1}}\left(\mathcal{L}_{s}\right)\right)+\cdots+\operatorname{deg}\left(S / I_{\rho_{c}}\left(\mathcal{L}_{s}\right)\right) .
$$

Therefore, it suffices to show that $\operatorname{deg}(S / P)=\operatorname{deg}\left(S / I_{\rho_{k}}\left(\mathcal{L}_{s}\right)\right)$ for $k=1, \ldots, c$. The ideal $I_{\rho_{k}}\left(\mathcal{L}_{s}\right)$ contains no monomials because it is a prime ideal. Let $\succ$ be the GRevLex order on $S$ and let $G=\left\{g_{1}, \ldots, g_{m}\right\}$ be the reduced Gröbner basis of $P$ with respect to $\succ$. We can write $g_{i}=t^{a_{i}^{+}}-t^{a_{i}^{-}}$for $i=1, \ldots, m$, with $\operatorname{in}\left(g_{i}\right)=t^{a_{i}^{+}}$. We set $g_{i}^{\prime}=t^{a_{i}^{+}}-\rho_{k}\left(a_{i}\right) t^{a_{i}^{-}}$and $G_{k}=\left\{g_{1}^{\prime}, \ldots, g_{m}^{\prime}\right\}$. Next, we show that $G_{k}$ is a Gröbner basis of $I_{\rho_{k}}\left(\mathcal{L}_{s}\right)$. Let $f \neq 0$ be an arbitrary (not necessarily pure) binomial of $I_{\rho_{k}}\left(\mathcal{L}_{s}\right)$. We claim that $f$ reduces to zero with respect to $G_{k}$ in the sense of $[11$, p. 23]. By the division algorithm (see [11, Theorem 2.11]), we can write

$$
f=h_{1} g_{1}^{\prime}+\cdots+h_{m} g_{m}^{\prime}+g
$$


where $\operatorname{in}(f) \succ \operatorname{in}\left(h_{i} g_{i}^{\prime}\right)$ for all $i$, and $g$ is a binomial (not necessarily pure) in $I_{\rho_{k}}\left(\mathcal{L}_{s}\right)$ such that none of the two terms of $g$ is divisible by any of the monomials $t^{a_{1}^{+}}, \ldots, t^{a_{m}^{+}}$. We can write

$$
g=\mu\left(t^{a}-\lambda t^{b}\right)=\mu t^{\delta}\left(t^{u^{+}}-\lambda t^{u^{-}}\right)
$$

with $\mu, \lambda \in K^{*}$ and $a-b=u^{+}-u^{-}$. As $t^{u^{+}}-\lambda t^{u^{-}}$is in $I_{\rho_{k}}\left(\mathcal{L}_{s}\right)$, it can be seen that $u=u^{+}-u^{-}$is in $\mathcal{L}_{s}$. Since $t^{u^{+}}-\rho_{k}(u) t^{u^{-}} \in I_{\rho_{k}}\left(\mathcal{L}_{s}\right)$, we get that $\lambda=\rho_{k}(u)$. If $g \neq 0$, we obtain that $t^{u^{+}}-t^{u^{-}}$, being in $P$, has one of its terms in the ideal $\operatorname{in}(P)=\left(t^{a_{1}^{+}}, \ldots, t_{m}^{a_{m}^{+}}\right)$, a contradiction. Thus, $g$ must be zero, i.e., $f$ reduces to zero with respect to $G_{k}$. This proves the claim. In particular, we obtain that $I_{\rho_{k}}\left(\mathcal{L}_{s}\right)$ is generated by $G_{k}$. To show that $G_{k}$ is a Gröbner basis, note that the S-polynomial of $g_{i}^{\prime}$ and $g_{j}^{\prime}$ is a binomial; thus, by the claim, it reduces to zero with respect to $G_{k}$. Therefore by Buchberger's criterion [9, Theorem 6, p. 84], $G_{k}$ is a Gröbner basis of $I_{\rho_{k}}\left(\mathcal{L}_{s}\right)$. Hence, $S / P$ and $S / I_{\rho_{k}}\left(\mathcal{L}_{s}\right)$ have the same degree.

Remark 4.7. Part (b) is well known. The proof given here is constructive and can be used - in one of the steps - to compute the degree of an arbitrary lattice ideal (see Example 4.12).

The program Normaliz [6] computes normalized volumes of lattice polytopes using polyhedral geometry. Thus we can compute the degree of any lattice ideal using Theorem 4.6. Next we give some applications and present some examples.

Corollary 4.8. If $d_{1}, \ldots, d_{s}$ are positive integers and $I$ is the toric ideal of $K\left[x_{1}^{d_{1}}, \ldots, x_{1}^{d_{s}}\right]$, then

$$
\operatorname{gcd}\left(d_{1}, \ldots, d_{s}\right) \operatorname{deg}(S / I)=\max \left\{d_{1}, \ldots, d_{s}\right\} .
$$

Proof. We may assume that $d_{1} \leq \cdots \leq d_{s}$. The order of $T\left(\mathbb{Z} /\left\langle d_{1}, \ldots, d_{s}\right\rangle\right)$ is $\operatorname{gcd}\left(d_{1}, \ldots, d_{s}\right)$. Then, by Theorem 4.5 , we get that $\operatorname{gcd}\left(d_{1}, \ldots, d_{s}\right) \operatorname{deg}(S / I)$ is $\operatorname{vol}\left(\left[0, d_{s}\right]\right)=d_{s}$.

Corollary 4.9. If $I(\mathcal{L})$ is a lattice ideal of dimension 1 which is homogeneous with respect to a positive vector $\left(d_{1}, \ldots, d_{s}\right)$, then

$$
\operatorname{gcd}\left(d_{1}, \ldots, d_{s}\right) \operatorname{deg}(S / I(\mathcal{L}))=\max \left\{d_{1}, \ldots, d_{s}\right\}\left|T\left(\mathbb{Z}^{s} / \mathcal{L}\right)\right| .
$$

Proof. Let $A$ be the $1 \times s$ matrix $\left(d_{1}, \ldots, d_{s}\right)$. By hypothesis $\mathcal{L} \subset \operatorname{ker}_{\mathbb{Z}}(A)$. The order of $T\left(\mathbb{Z} /\left\langle d_{1}, \ldots, d_{s}\right\rangle\right)$ is $\operatorname{gcd}\left(d_{1}, \ldots, d_{s}\right)$. Then, by Theorem 4.6 , we get

$$
\operatorname{deg}(S / I(\mathcal{L}))=\frac{T\left(\mathbb{Z}^{s} / \mathcal{L}\right) \operatorname{vol}\left(\operatorname{conv}\left(0, d_{1}, \ldots, d_{s}\right)\right)}{T\left(\mathbb{Z} /\left\langle d_{1}, \ldots, d_{s}\right\rangle\right)}=\frac{\left|T\left(\mathbb{Z}^{s} / \mathcal{L}\right)\right| \max \left\{d_{1}, \ldots, d_{s}\right\}}{\operatorname{gcd}\left(d_{1}, \ldots, d_{s}\right)} .
$$

For 1-dimensional lattice ideals that are not necessarily homogeneous, we can express the degree in terms of a basis of the lattice (see Example 8.14).

Corollary 4.10. Let $\mathcal{L} \subset \mathbb{Z}^{s}$ be a lattice of rank $s-1$ and let $\alpha_{1}, \ldots, \alpha_{s-1}$ be $a \mathbb{Z}$-basis of $\mathcal{L}$. If $\alpha_{i}=\left(\alpha_{1, i}, \ldots, \alpha_{s, i}\right)$, for $i=1, \ldots, s-1$, and

$$
v_{i}=(-1)^{i} \operatorname{det}\left(\begin{array}{ccc}
\alpha_{1,1} & \ldots & \alpha_{1, s-1} \\
\vdots & & \vdots \\
\alpha_{i-1,1} & \ldots & \alpha_{i-1, s-1} \\
\alpha_{i+1,1} & \ldots & \alpha_{i+1, s-1} \\
\vdots & & \vdots \\
\alpha_{s, 1} & \ldots & \alpha_{s, s-1}
\end{array}\right) \text {, for } i=1, \ldots, s,
$$

then $\operatorname{deg}(S / I(\mathcal{L}))=\max \left\{v_{1}, \ldots, v_{s}, 0\right\}-\min \left\{v_{1}, \ldots, v_{s}, 0\right\}$. 
Proof. Let $B$ be the $s \times(s-1)$ matrix with columns $\alpha_{1}, \ldots, \alpha_{s-1}$ and let $A$ be the $1 \times s$ matrix $\left(v_{1}, \ldots, v_{s}\right)$. We set $r=s-1$. The order of $T\left(\mathbb{Z}^{s} / \mathcal{L}\right)$ is equal to $\operatorname{gcd}\left(v_{1}, \ldots, v_{s}\right)$, the gcd of the $r \times r$ minors of $B$. The order of $T\left(\mathbb{Z} /\left\langle v_{1}, \ldots, v_{s}\right\rangle\right)$ is also equal to $\operatorname{gcd}\left(v_{1}, \ldots, v_{s}\right)$. Since $A B=0$, we obtain that $\mathcal{L} \subset \operatorname{ker}(A)$. Hence, by Theorem 4.6, we get that $\operatorname{deg}(S / I(\mathcal{L}))$ is equal to $\operatorname{vol}\left(\operatorname{conv}\left(0, v_{1}, \ldots, v_{s}\right)\right)$ which is equal to $\max \left\{v_{1}, \ldots, v_{s}, 0\right\}-\min \left\{v_{1}, \ldots, v_{s}, 0\right\}$.

The next examples illustrate how to use Theorems 4.5 and 4.6 to compute the degree.

Example 4.11. Let $P$ be the toric ideal of the monomial subring

$$
K[H]=K\left[x_{2} x_{1}^{-1}, x_{3} x_{2}^{-1}, x_{4} x_{3}^{-1}, x_{1} x_{4}^{-1}, x_{5} x_{2}^{-1}, x_{3} x_{5}^{-1}, x_{4} x_{5}^{-1}\right] .
$$

We employ the notation of Theorem 4.5. The rank of $A$ is 4 and the height of $P$ is 3 . Using Normaliz [6], we get

$$
4 ! \operatorname{vol}\left(\operatorname{conv}\left(v_{1}, \ldots, v_{7}, 0\right)\right)=11 .
$$

As the group $\mathbb{Z}^{5} /\left\langle v_{1}, \ldots, v_{7}\right\rangle$ is torsion free, by Theorem 4.5 we have that the degree of $K\left[t_{1}, \ldots, t_{7}\right] / P$ is equal to 11 .

Example 4.12. Let $K$ be the field of rational numbers and let $\mathcal{L}$ be the lattice of rank 4 generated by

$$
\begin{array}{ll}
a_{1}=(2,1,1,1,-1,-1,-1,-2), & a_{2}=(1,1,-1,-1,1,1,-1,-1), \\
a_{3}=(2,-1,1,-2,1,-1,1,-1), & a_{4}=(5,-5,0,0,0,0,0,0) .
\end{array}
$$

We use the notation of Theorem 4.6. The vectors $a_{1}, \ldots, a_{4}, e_{1}, e_{3}, e_{4}, e_{5}$ form a $\mathbb{Q}$ basis of $\mathbb{Q}^{8}$. In this case we obtain the matrix

$$
A=\left(\begin{array}{rrrrrrrr}
4 & 4 & 0 & 0 & 0 & -1 & 1 & 6 \\
0 & 0 & 1 & 0 & 0 & 1 & 0 & 0 \\
0 & 0 & 0 & 4 & 0 & 7 & 9 & -6 \\
0 & 0 & 0 & 0 & 2 & -3 & -3 & 2
\end{array}\right)
$$

whose columns are denoted by $v_{1}, \ldots, v_{8}$. Let $P$ be the toric ideal of $K\left[x^{v_{1}}, \ldots, x^{v_{8}}\right]$. Therefore, by Theorem 4.6, we get

$$
\operatorname{deg}(S / I(\mathcal{L}))=\left|T\left(\mathbb{Z}^{8} / \mathcal{L}\right)\right| \operatorname{deg}(S / P)=\frac{5(4) ! \operatorname{vol}\left(\operatorname{conv}\left(0, v_{1}, \ldots, v_{8}\right)\right)}{\left|T\left(\mathbb{Z}^{4} /\left\langle v_{1}, \ldots, v_{8}\right\rangle\right)\right|}=\frac{(5)(200)}{8} .
$$

Thus $\operatorname{deg}(S / I(\mathcal{L}))=125$. The normalized volume of the polytope $\operatorname{conv}\left(0, v_{1}, \ldots, v_{8}\right)$ was computed using Normaliz [6].

5. Primary decompositions of homogeneous binomial ideals. In this part we present some applications of the main result of section 3 to the theory of graded binomial ideals and graded lattice ideals of dimension 1 . We continue to employ the notation and definitions used in section 2.

Given a subset $I \subset S$, its variety, denoted by $V(I)$, is the set of all $a \in \mathbb{A}_{K}^{s}$ such that $f(a)=0$ for all $f \in I$, where $\mathbb{A}_{K}^{s}$ denotes an affine $s$-space over $K$. In this section we focus on homogeneous binomial ideals $I$ of $S$ with $V\left(I, t_{i}\right)=\{0\}$ for all $i$.

LEMma 5.1. Let $I$ be a homogeneous binomial ideal of $S$ such that $V\left(I, t_{i}\right)=\{0\}$ for all $i$. Then, for any associated prime $\mathfrak{p}$ of $I$, either $\mathfrak{p}=\mathfrak{m}$, or else $\mathfrak{p} \subsetneq \mathfrak{m}, t_{i} \notin \mathfrak{p}$ for all $i$, and $\operatorname{ht}(\mathfrak{p})=s-1$. Moreover, $\operatorname{ht}(I)=s-1$.

Proof. Since $I$ is homogeneous, any associated prime $\mathfrak{p}$ of $I$ is homogeneous too. Hence $I \subset \mathfrak{p} \subset \mathfrak{m}$. In particular, $\left(I, t_{1}\right) \subset \mathfrak{m}$. Let $\mathfrak{q}$ be any minimal prime ideal over $\left(I, t_{1}\right)$. By [20, Lemma 2.6], $\mathfrak{q}=\mathfrak{m}$ and so $s=\mathrm{ht}\left(I, t_{1}\right) \leq \mathrm{ht}(I)+1$ (here we use the 
fact that $I$ is homogeneous). Thus $h t(I) \geq s-1$. Suppose that $\mathfrak{p}$ is an associated prime of $I, \mathfrak{p} \neq \mathfrak{m}$. Then $t_{i} \notin \mathfrak{p}$ for all $i$, because if some $t_{i} \in \mathfrak{p},\left(I, t_{i}\right) \subset \mathfrak{p}$, and by $[20$, Lemma 2.6] again, $\mathfrak{p}$ would be equal to $\mathfrak{m}$. In particular, $\operatorname{ht}(\mathfrak{p})=s-1$. Finally ht $(I)=s-1$, otherwise $\mathfrak{m}$ would be the only associated prime of $I$. Thus $\mathfrak{m}=\operatorname{rad}(I)$, a contradiction because $I$ cannot contain a power of $t_{i}$ for any $i=1, \ldots, s$.

The assumption that $I$ is homogeneous in the result above is crucial.

Example 5.2. Let $S=K\left[t_{1}, t_{2}, t_{3}\right]$ and $I=\left(t_{1} t_{2} t_{3}-1\right) \mathfrak{m}$. Clearly $V\left(I, t_{i}\right)=\{0\}$ for all $i$. However, $\operatorname{ht}(I)=1$ and $\operatorname{ht}\left(I, t_{i}\right)=3$ for all $i$.

An ideal $I$ is said to be unmixed if all of its associated primes have the same height (see, e.g., [38, p. 196]). We write $\operatorname{Hull}(I)$ for the intersection of the isolated primary components of $I$.

Proposition 5.3. Let I be a graded binomial ideal of $S$ such that $V\left(I, t_{i}\right)=\{0\}$ for all $i$. The following conditions are equivalent:

(a) $I$ is a lattice ideal; (b) $I=\left(I:\left(t_{1} \cdots t_{s}\right)^{\infty}\right)$; (c) $t_{i}$ is regular on $S / I$ for all $i$;

(d) $I=\left(I: t_{1}\right)$; (e) $I$ is Cohen-Macaulay; (f) $I$ is unmixed; (g) $I=\operatorname{Hull}(I)$.

Proof. The equivalences among (a), (b), and (c) follow from Theorem 2.9. By Lemma 5.1, (f) and (g) are equivalent. Clearly (c) $\Rightarrow(\mathrm{d})$. By Lemma 5.1, ht $(I)=s-1$ and, for any associated prime $\mathfrak{p}$ of $I$, either $\mathfrak{p}=\mathfrak{m}$, or else $\mathfrak{p} \subsetneq \mathfrak{m}, \operatorname{ht}(\mathfrak{p})=s-1$, and $t_{1} \cdots t_{s} \notin \mathfrak{p}$. Therefore $I$ has a minimal primary decomposition either of the form $I=\mathfrak{q}_{1} \cap \cdots \cap \mathfrak{q}_{c}=\operatorname{Hull}(I)$, or else $I=\mathfrak{q}_{1} \cap \cdots \cap \mathfrak{q}_{c} \cap \mathfrak{q}=\operatorname{Hull}(I) \cap \mathfrak{q}$, where the $\mathfrak{q}_{i}$ are $\mathfrak{p}_{i}$-primary ideals with $\operatorname{ht}\left(\mathfrak{p}_{i}\right)=s-1$, and $\mathfrak{q}$ is $\mathfrak{m}$-primary. Therefore (e) and (f) are equivalent. Moreover, either $\left(I: t_{1}^{\infty}\right)=\cap_{i=1}^{c}\left(\mathfrak{q}_{i}: t_{1}^{\infty}\right)=\cap_{i=1}^{c} \mathfrak{q}_{i}=\operatorname{Hull}(I)$, or else $\left(I: t_{1}^{\infty}\right)=\cap_{i=1}^{c}\left(\mathfrak{q}_{i}: t_{1}^{\infty}\right) \cap\left(\mathfrak{q}: t_{1}^{\infty}\right)=\cap_{i=1}^{c} \mathfrak{q}_{i}=\operatorname{Hull}(I)$, since $\left(\mathfrak{q}: t_{1}^{\infty}\right)=S$. In both cases, $\operatorname{Hull}(I)=\left(I: t_{1}^{\infty}\right)$.

Suppose (d) holds. Then $I=\left(I: t_{1}\right)=\left(I: t_{1}^{\infty}\right)=\operatorname{Hull}(I)$ and $I$ is unmixed. Thus (d) $\Rightarrow(\mathrm{f})$. Suppose (f) holds. Then $\mathfrak{m}$ is not an associated prime of $I$, and, by Lemma $5.1, t_{i}$ cannot be in any associated prime of $I$, so each $t_{i}$ is regular modulo $I$ and (c) holds.

DeFINITION 5.4. Let $L$ be an $s \times m$ integer matrix, let $l_{*, i}$ be the ith column of $L$, and let $f_{i}=t_{*, i}^{l_{*}^{+}}-t_{*, i}^{l_{*}^{-}}$be the binomial of $S=K\left[t_{1}, \ldots, t_{s}\right]$ defined by $l_{*, i}$. We call $I(L):=\left(f_{1}, \ldots, f_{m}\right)$ the matrix ideal associated with $L$.

Whenever $I(L)$ is graded with respect to a weight vector $\mathbf{d}=\left(d_{1}, \ldots, d_{s}\right) \in \mathbb{N}_{+}^{s}$, we can and will suppose, without loss of generality, that $\operatorname{gcd}(\mathbf{d})=1$.

Definition 5.5 (see [30, p. 397]). Let $\mathbf{d}=\left(d_{1}, \ldots, d_{s}\right) \in \mathbb{N}_{+}^{s}$. The Herzog ideal associated with $\mathbf{d}$, denote by $\mathfrak{p}_{\mathbf{d}}$, is the toric ideal of $K\left[x_{1}^{d_{1}}, \ldots, x_{1}^{d_{s}}\right]$, where $x_{1}$ is a variable.

Remark 5.6. Let $I(L)=\left(f_{1}, \ldots, f_{m}\right)$ be the matrix ideal associated with an $s \times m$ integer matrix $L$. The following conditions are equivalent:

(a) there exists an $\mathbb{N}$-grading of $S$, with each $t_{i}$ of weight $d_{i}>0$, under which each $f_{i}$ is homogeneous of positive degree;

(b) there exists $\mathbf{d}=\left(d_{1}, \ldots, d_{s}\right) \in \mathbb{N}_{+}^{s}$ such that $\mathbf{d} L=0$;

(c) there exists $\mathbf{d}=\left(d_{1}, \ldots, d_{s}\right) \in \mathbb{N}_{+}^{s}$ with $I(L) \subset \mathfrak{p}_{\mathbf{d}}$, the Herzog ideal associated with $\mathbf{d}$.

We give now a result on the structure of graded matrix ideals.

Proposition 5.7. Let $I$ be the matrix ideal of an $s \times m$ integer matrix $L$ and let $\mathcal{L}$ be the lattice spanned by the columns of $L$. Suppose that $I$ is graded and that $V\left(I, t_{i}\right)=\{0\}$ for all $i$. Then we have the following.

(a) I has a minimal primary decomposition either of the form $I=\mathfrak{q}_{1} \cap \cdots \cap \mathfrak{q}_{c}$, if $I$ is unmixed, or else $I=\mathfrak{q}_{1} \cap \cdots \cap \mathfrak{q}_{c} \cap \mathfrak{q}$, if I is not unmixed, where the $\mathfrak{q}_{i}$ are $\mathfrak{p}_{i}$-primary ideals with $\mathrm{ht}\left(\mathfrak{p}_{i}\right)=s-1$, and $\mathfrak{q}$ is an $\mathfrak{m}$-primary ideal. 
(b) For all $g \in \mathfrak{m} \backslash \cup_{i=1}^{c} \mathfrak{p}_{i}, I(\mathcal{L})=\left(I:\left(t_{1} \cdots t_{s}\right)^{\infty}\right)=\left(I: g^{\infty}\right)=\mathfrak{q}_{1} \cap \cdots \cap \mathfrak{q}_{c}=$ $\operatorname{Hull}(I)$.

(c) $\operatorname{rank}(L)=s-1$ and there exists a unique Herzog ideal $\mathfrak{p}_{\mathbf{d}}$ containing $I$.

(d) If $I$ is not unmixed and $h=t_{1} \cdots t_{s}$, there exists $a \in \mathbb{N}_{+}$such that $I(\mathcal{L})=$ $\left(I: h^{a}\right), \mathfrak{q}=I+\left(h^{a}\right)$ is an irredundant $\mathfrak{m}$-primary component of $I$, and $I=$ $I(\mathcal{L}) \cap \mathfrak{q}$.

(e) Either $c \leq\left|T\left(\mathbb{Z}^{s} / \mathcal{L}\right)\right|$, if $\operatorname{char}(K)=0$, or else $c \leq|G|$, if $\operatorname{char}(K)=p, p$ a prime, where $G$ is the unique largest subgroup of $T\left(\mathbb{Z}^{s} / \mathcal{L}\right)$ whose order is relatively prime to $p$. If $K$ is algebraically closed, then equality holds.

Proof. We set $h=t_{1} \cdots t_{s}$. Item ( $a$ ) follows from Lemma 5.1 (see the proof of Proposition 5.3). By Lemma 2.10, $I(\mathcal{L})=\left(I: h^{\infty}\right)$, which proves the first equality in (b). Let $g \in \mathfrak{m} \backslash \cup_{i=1}^{c} \mathfrak{p}_{i}$. Suppose that $I=\mathfrak{q}_{1} \cap \cdots \cap \mathfrak{q}_{c}$. Then, for any $a \in \mathbb{N}_{+}$, we have $\left(I: g^{a}\right)=\cap_{j=1}^{c}\left(\mathfrak{q}_{j}: g^{a}\right)=\cap_{j=1}^{c} \mathfrak{q}_{j}$, because $g^{a} \notin \mathfrak{p}_{j}$ and $\mathfrak{q}_{j}$ is $\mathfrak{p}_{j}$-primary. Suppose that $I=\mathfrak{q}_{1} \cap \cdots \cap \mathfrak{q}_{c} \cap \mathfrak{q}$. Then, for $a \gg 0,\left(I: g^{a}\right)=\left(I: g^{\infty}\right)$ and $g^{a} \in \mathfrak{m}^{a} \subset \mathfrak{q}$, so that $\left(\mathfrak{q}: g^{a}\right)=S$. Thus $\left(I: g^{a}\right)=\cap_{j=1}^{c}\left(\mathfrak{q}_{j}: g^{a}\right) \cap\left(\mathfrak{q}: g^{a}\right)=\cap_{j=1}^{c} \mathfrak{q}_{j}$. In either case, one has the equality $\left(I: g^{\infty}\right)=\mathfrak{q}_{1} \cap \cdots \cap \mathfrak{q}_{c}$, which coincides with Hull $(I)$. By a similar calculation, we get $\left(I: h^{\infty}\right)=\mathfrak{q}_{1} \cap \cdots \cap \mathfrak{q}_{c}$.

In particular, using $(b), \operatorname{ht}(I)=\operatorname{ht}(I(\mathcal{L}))$. Since $\operatorname{ht}(I(\mathcal{L}))=\operatorname{rank}(\mathcal{L})=\operatorname{rank}(L)$, it follows that $\operatorname{rank}(L)=s-1$. Since $I$ is homogeneous, by Remark 5.6, there exists $\mathbf{d} \in \mathbb{N}_{+}^{s}$ such that $I \subset \mathfrak{p}_{\mathbf{d}}$, the Herzog ideal associated with $\mathbf{d}$. Since $\operatorname{rank}\left(L^{\top}\right)=$ $\operatorname{rank}(L)=s-1$, then $\operatorname{ker}\left(L^{\top}\right)$ is generated as a $\mathbb{Q}$-linear subspace by $\mathbf{d}^{\top}$. Thus $\mathfrak{p}_{\mathbf{d}}$ is the unique Herzog ideal containing $I$ (see [30, Remark 3.2]). This proves (c).

Suppose that $I$ is not unmixed. As $S$ is a Noetherian ring, there exists $a \in \mathbb{N}_{+}$ such that $\left(I: h^{a}\right)=\left(I: h^{\infty}\right)$. By [10, Proposition 7.2], $I=\left(I: h^{a}\right) \cap\left(I+\left(h^{a}\right)\right)$, where $\left(I: h^{a}\right)=I(\mathcal{L})$ is the hull of $I$. Since $I$ is not unmixed, $I+\left(h^{a}\right)$ must be irredundant. Moreover, by [20, Lemma 2.6], the only prime ideal containing $I+\left(h^{a}\right)$ is $\mathfrak{m}$. It follows that $I+\left(h^{a}\right)$ is $\mathfrak{m}$-primary. This proves $(d)$. Finally, $(e)$ follows from Theorem $3.2(\mathrm{~b})$.

Let $I$ be the matrix ideal associated with an $s \times m$ integer matrix $L$. The conditions $I$ homogeneous and $\operatorname{rank}(L)=s-1$ do not imply that $V\left(I, t_{i}\right)=\{0\}$ for all $i$.

Example 5.8. Let $L$ be the matrix with column vectors $(2,-2,0)$ and $(-2,1,1)$ and let $I$ be its matrix ideal $\left(t_{1}^{2}-t_{2}^{2}, t_{1}^{2}-t_{2} t_{3}\right)$. Clearly $I$ is homogeneous (with the standard grading) and $\operatorname{rank}(L)=2$. However $(0,0, \lambda) \in V\left(I, t_{1}\right)$ for any $\lambda \in K$.

As a consequence of the previous result, we obtain the following.

Corollary 5.9. Let $I=I(L)$ be the matrix ideal associated with an $s \times m$ integer matrix $L$. Let $\mathcal{L}$ be the lattice spanned by the columns of $L$. Suppose that $I$ is homogeneous with respect to a weight vector $\mathbf{d}=\left(d_{1}, \ldots, d_{s}\right) \in \mathbb{N}_{+}^{s}$ and that $V\left(I, t_{i}\right)=\{0\}$ for all $i$. Then

$$
\operatorname{deg}(S / I)=\max \left\{d_{1}, \ldots, d_{s}\right\}\left|T\left(\mathbb{Z}^{s} / \mathcal{L}\right)\right|=\max \left\{d_{1}, \ldots, d_{s}\right\} \Delta_{s-1}(L) .
$$

Proof. By Proposition 5.7, $\operatorname{Hull}(I)=\mathfrak{q}_{1} \cap \cdots \cap \mathfrak{q}_{c}=I(\mathcal{L})$. Thus, by Proposition 2.5,

$$
\operatorname{deg}(S / I)=\sum_{j=1}^{c} \operatorname{deg}\left(S / \mathfrak{q}_{j}\right)=\operatorname{deg}(S / I(\mathcal{L}))
$$

By Corollary 4.9, we have $\operatorname{deg}(S / I(\mathcal{L}))=\max \left\{d_{1}, \ldots, d_{s}\right\}\left|T\left(\mathbb{Z}^{s} / \mathcal{L}\right)\right|$. To complete the proof notice that, by Theorem 2.13 , one has $\left|T\left(\mathbb{Z}^{s} / \mathcal{L}\right)\right|=\Delta_{s-1}(L)$.

6. Generalized positive critical binomial ideals. We continue to employ the notation and definitions used in section 1 . In this section we restrict ourselves to 
the study of certain classes of square integer matrices (see Definition 1.1) and their corresponding matrix ideals.

Recall that the support of a polynomial $f \in S$, denoted by $\operatorname{supp}(f)$, is defined as the set of all variables $t_{i}$ that occur in $f$.

Proposition 6.1. Let $I=I(L)=\left(f_{1}, \ldots, f_{s}\right)$ be the matrix ideal associated with an $s \times s P B$ matrix L. Suppose that $\left|\operatorname{supp}\left(f_{j}\right)\right| \geq 4$, for all $j=1, \ldots, s$. Then

(a) $I$ is not a lattice ideal;

(b) if $V\left(I, t_{i}\right)=\{0\}$ for all $i$, then $\mathfrak{m}$ is an associated prime of $I$;

(c) if $I$ is graded and $V\left(I, t_{i}\right)=\{0\}$ for all $i$, then $I$ has a minimal primary decomposition of the form $I=\mathfrak{q}_{1} \cap \cdots \cap \mathfrak{q}_{c} \cap \mathfrak{q}$, where the $\mathfrak{q}_{i}$ are $\mathfrak{p}_{i}$-primary ideals with $\mathrm{ht}\left(\mathfrak{p}_{i}\right)=s-1$, and $\mathfrak{q}=\left(I,\left(t_{1} \cdots t_{s}\right)^{a}\right)$ is an $\mathfrak{m}$-primary ideal, for some $a \in \mathbb{N}_{+}$. Moreover, I has at most $\left|T\left(\mathbb{Z}^{s} / \mathcal{L}\right)\right|+1$ primary components.

Proof. Assume that $I$ is a lattice ideal. There is $k>1$ such that $f_{1}=t_{1}^{a_{1,1}}-$ $t_{k}^{a_{k, 1}} \cdots t_{s}^{a_{s, 1}}$ and $f_{k}=t_{k}^{a_{k, k}}-t_{1}^{a_{1, k}} \cdots t_{k-1}^{a_{k-1, k}} t_{k+1}^{a_{k+1, k}} \cdots t_{s}^{a_{s, k}}$ with $a_{k, 1}>0$. We claim that $a_{1,1}>a_{1, k}$. If $a_{1,1} \leq a_{1, k}$, then from the equality

$$
\begin{aligned}
& \left(t_{1}^{a_{1,1}}-t_{k}^{a_{k, 1}} \cdots t_{s}^{a_{s, 1}}\right) t_{1}^{a_{1, k}-a_{1,1}} t_{2}^{a_{2, k}} \cdots t_{k-1}^{a_{k-1, k}} t_{k+1}^{a_{k+1, k}} \cdots t_{s}^{a_{s, k}} \\
& \quad+\left(t_{k}^{a_{k, k}}-t_{1, k}^{a_{1, k}} \cdots t_{k-1}^{a_{k-1, k}} t_{k+1}^{a_{k+1, k}} \cdots t_{s}^{a_{s, k}}\right) \\
& \quad=t_{k}^{a_{k, k}}-t_{1}^{a_{1, k}-a_{1,1}} t_{2}^{a_{2, k}} \cdots t_{k-1}^{a_{k-1, k}} t_{k}^{a_{k, 1}} t_{k+1}^{a_{k+1, k}+a_{k+1,1}} \cdots t_{s}^{a_{s, k}+a_{s, 1}}=: t_{k} g \neq 0
\end{aligned}
$$

one has $t_{k} g \in I$. As $t_{k}$ is a nonzero divisor of $S / I$, we get $g \in I$. Thus, $g$ is a linear combination with coefficients in $S$ of $f_{1}, \ldots, f_{s}$. Since $t_{k}^{a_{k, k}-1}$ is a term of $g$, we conclude that $t_{k}^{a_{k, k}-1}$ is a multiple of some term of $f_{i}$ for some $i \neq k$, a contradiction to the fact that, a fortiori, $\operatorname{supp}\left(f_{i}\right)$ has at least 3 variables. This proves $a_{1,1}>a_{1, k}$. Moreover, from the equality

$$
\begin{aligned}
& \left(t_{k}^{a_{k, k}}-t_{1}^{a_{1, k}} \cdots t_{k-1}^{a_{k-1, k}} t_{k+1}^{a_{k+1, k}} \cdots t_{s}^{a_{s, k}}\right) t_{1}^{a_{1,1}-a_{1, k}} \\
& \quad+\left(t_{1,1}^{a_{1,1}}-t_{k}^{a_{k, 1}} \cdots t_{s}^{a_{s, 1}}\right) t_{2}^{a_{2, k}} \cdots t_{k-1}^{a_{k-1, k}} t_{k+1}^{a_{k+1, k}} \cdots t_{s}^{a_{s, k}} \\
& \quad=t_{k}^{a_{k, k}} t_{1}^{a_{1,1}-a_{1, k}}-t_{2}^{a_{2, k}} \cdots t_{k-1}^{a_{k-1, k}} t_{k}^{a_{k, 1}} t_{k+1}^{a_{k+1, k}+a_{k+1,1}} \cdots t_{s}^{a_{s, k}+a_{s, 1}}=: t_{k} g^{\prime} \neq 0
\end{aligned}
$$

the argument above shows that $t_{k}^{a_{k, k}-1} t_{1}^{a_{1,1}-a_{1, k}}$ is a multiple of some term of $f_{i}$ for some $i$, a contradiction to the fact that $\operatorname{supp}\left(f_{i}\right)$ has at least 4 variables. Hence $I$ is not a lattice ideal.

Since $I$ is not a lattice ideal, $t_{i}$ is a zero divisor of $S / I$ for some $i$. Then some associated prime $\mathfrak{p}$ of $I$ contains $t_{i}$. Hence, by [20, Lemma 2.6], $\mathfrak{p}$ is equal to $\mathfrak{m}$, which proves (b). Since $I$ is not a lattice ideal, $I$ is not unmixed (see Proposition 5.3). The rest follows on applying Proposition 5.7.

Proposition 6.2. Let $g_{1}, \ldots, g_{s}$ be the binomials defined by the rows of a $G C B$ matrix $L$ and let $I$ be the ideal generated by $g_{1}, \ldots, g_{s}$. If $V\left(I, t_{i}\right)=\{0\}$ and $\left|\operatorname{supp}\left(g_{i}\right)\right| \geq 3$ for all $i$, then $I$ is not a complete intersection.

Proof. We may assume that $g_{i}$ corresponds to the $i$ th row of $L$. We proceed by contradiction. Assume that $I$ is a complete intersection. As $I$ is graded, since $L$ is a GCB matrix, and the height of $I$ is $s-1$, we may assume that $g_{1}, \ldots, g_{s-1}$ generate $I$. Hence, we can write

$$
g_{s}=t_{s}^{a_{s, s}}-t_{1}^{a_{s, 1}} \cdots t_{s-1}^{a_{s, s-1}}=h_{1} g_{1}+\cdots+h_{s-1} g_{s-1}
$$

for some $h_{1}, \ldots, h_{s-1}$ in $S$, where $a_{s, s}>0$. Therefore, the monomial $t_{s}^{a_{s, s}}$ has to occur in the right-hand side of this equation, a contradiction to the fact that $\left|\operatorname{supp}\left(g_{i}\right)\right| \geq 3$ for all $i$. 
In what follows, we examine GPCB matrices - a natural extension of the $\mathrm{PCB}$ matrices introduced in [30] - and the algebra of their matrix ideals. The class of GPCB matrices is closed under transposition, as the next result shows.

THEOREM 6.3. Let $L$ be an integer matrix of size $s \times s$ with rows $\ell_{1}, \ldots, \ell_{s}$ and let $\left(L_{i, j}\right)$ be the adjoint matrix of $L$. Suppose $L \mathbf{b}^{\top}=0$ for some $\mathbf{b}$ in $\mathbb{N}_{+}^{s}$. The following hold.

(a) If $\operatorname{rank}\left(\ell_{1}, \ldots, \widehat{\ell}_{i}, \ldots, \ell_{s}\right)=s-1$ and $L_{i, i} \geq 0$ for all $i$, then $\mathbf{c} L=0$ for some $\mathbf{c} \in \mathbb{N}_{+}^{s}$.

(b) If $L_{i, i}>0$ for all $i$, then $\mathbf{c} L=0$ for some $\mathbf{c} \in \mathbb{N}_{+}^{s}$.

(c) If $L$ is a GCB matrix and $\operatorname{rank}\left(\ell_{1}, \ldots, \widehat{\ell}_{i}, \ldots, \ell_{s}\right)=s-1$ for all $i$, then $L^{\top}$ is a $G C B$ matrix.

(d) If $L$ is a GPCB matrix, then $\operatorname{rank}(L)=s-1$ and $L^{\top}$ is a GPCB matrix.

(e) If $s=3$ and $L$ is a GCB matrix, then $\operatorname{rank}(L)=2$ and $L^{\top}$ is a GCB matrix.

Proof.

(a) Let $L_{i}$ be the $i$ th column of $\operatorname{adj}(L)=\left(L_{i, j}\right)$. Since $\ell_{1}, \ldots, \widehat{\ell}_{i}, \ldots, \ell_{s}$ are linearly independent, we get $L_{i} \neq 0$. The vector $\mathbf{b}$ generates $\operatorname{ker}_{\mathbb{Q}}(L)$ because $L$ has rank $s-1$ and $L \mathbf{b}^{\top}=0$. Then, because of the equality $\operatorname{Ladj}(L)=0$, we can write $L_{i}=\mu_{i} \mathbf{b}$ for some $\mu_{i} \in \mathbb{Q}$. Notice that $\mu_{i}>0$ because $L_{i, i} \geq 0$ and $\mathbf{b} \in \mathbb{N}_{+}^{s}$. Hence, all entries of $\operatorname{adj}(L)$ are positive integers. If $\mathbf{c}$ is any row of $\operatorname{adj}(L)$, we get $\mathbf{c} L=0$ because $\operatorname{adj}(L) L=0$.

(b) For any $i$, the vectors $\ell_{1}, \ldots, \widehat{\ell}_{i}, \ldots, \ell_{s}$ are linearly independent because $L_{i, i}>$ 0 . Thus this part follows from (a).

(c) Let $L$ be a GCB matrix as in Definition 1.1, (1.1). ( $\left.\mathrm{c}_{1}\right)$ First we treat the case $\mathbf{b}=\mathbf{1}=(1, \ldots, 1)$, i.e., the case where $L$ is a $\mathrm{CB}$ matrix. By part (a) it suffices to show that $L_{i, i} \geq 0$ for all $i$. Let $H_{i, i}$ be the submatrix of $L$ obtained by eliminating the $i$ th row and $i$ th column. By the Gershgorin circle theorem, every (possibly complex) eigenvalue $\lambda$ of $H_{i, i}$ lies within at least one of the discs $\left\{z \in \mathbb{C} \mid\left\|z-a_{j, j}\right\| \leq r_{j}\right\}, j \neq i$, where $r_{j}=\sum_{u \neq i, j}\left|-a_{j, u}\right| \leq a_{j, j}$ since $L \mathbf{1}^{\top}=0$ and $a_{i, j} \geq 0$ for all $i, j$. If $\lambda \in \mathbb{R}$, we get $\left|\lambda-a_{j, j}\right| \leq a_{j, j}$, and, consequently, $\lambda \geq 0$. If $\lambda \notin \mathbb{R}$, then since $H_{i, i}$ is a real matrix, its conjugate $\bar{\lambda}$ must also be an eigenvalue of $H_{i, i}$. Since $\operatorname{det}\left(H_{i, i}\right)$ is the product of the $s-1$ (possibly repeated) eigenvalues of $H_{i, i}$, we get $L_{i, i} \geq 0$. This argument is adapted from the proof of [30, Lemma 2.1]. $\left(\mathrm{c}_{2}\right)$ Now, we treat the general case. Let $B$ be the $s \times s$ diagonal matrix $\operatorname{diag}\left(b_{1}, \ldots, b_{s}\right)$, where $\mathbf{b}=\left(b_{1}, \ldots, b_{s}\right)$, and let $\widetilde{L}=L B$. Notice that $\widetilde{L} \mathbf{1}^{\top}=0$ because $L \mathbf{b}^{\top}=0$, and $\widetilde{L}$ is a CB matrix because $L$ is a GCB matrix. Let $\left(\widetilde{L}_{i, j}\right)$ be the adjoint matrix of $\widetilde{L}$. Since $b_{i}>0$ for all $i$, by the multilinearity of the determinant, it follows that $L_{i, j} \neq 0$ if and only if $\widetilde{L}_{i, j} \neq 0$. Hence, any set of $s-1$ rows of $\widetilde{L}$ is linearly independent. Therefore, applying case $\left(c_{1}\right)$ to $\widetilde{L}$, we obtain that $\widetilde{L}^{\top}$ is a GCB matrix. Thus, there is $\mathbf{c} \in \mathbb{N}_{+}^{s}$ such that $\mathbf{c} \widetilde{L}=0$. Then, c $L=0$, i.e., $L^{\top}$ is a GCB matrix, as required.

(d) Let $L$ be a GPCB matrix. By the argument given in $\left(\mathrm{c}_{1}\right)$, it follows readily that $L_{i, i}>0$ for all $i$. In particular the rank of $L$ is $s-1$. Hence, by part (b), $L^{\top}$ is a GPCB matrix.

(e) Let $L$ be a GCB matrix with $s=3$. It is easy to see that $L_{i, j}>0$ for $i \neq j$. In particular $L$ has rank 2 and any two rows of $L$ are linearly independent. Then by (c), $L^{\top}$ is a GCB matrix.

Following the proof of (c) above, we call $\widetilde{L}:=L B$ the associated $\mathrm{CB}$ matrix of $L$. 
Lemma 6.4. Let $L$ be a $G C B$ matrix of size $s \times s$. If $\operatorname{rank}(L)=s-1$, then any set of $s-1$ columns is linearly independent.

Proof. Let $\ell_{*, 1}, \ldots, \ell_{*, s}$ be the columns of the matrix $L$. By hypothesis, there is a vector $\mathbf{b}=\left(b_{1}, \ldots, b_{s}\right) \in \mathbb{N}_{+}^{s}$ such that $L \mathbf{b}^{\top}=0$. Thus it suffices to observe that $b_{1} \ell_{*, 1}+\cdots+b_{s} \ell_{*, s}=0$.

Example 6.5 . Let $L$ be the $4 \times 4$ integer matrix of rank 3 :

$$
L=\left(\begin{array}{rrrr}
5 & -2 & 0 & -1 \\
0 & 1 & -1 & 0 \\
0 & -1 & 1 & 0 \\
-1 & 0 & -1 & 4
\end{array}\right)
$$

Then $L$ is a GCB matrix, where $L \mathbf{b}^{\top}=0$ if $\mathbf{b}=(9,19,19,7)$ but $L^{\top}$ is not a GCB matrix because rows 2,3 , and 4 are linearly dependent (see Lemma 6.4).

Remark 6.6. Observe that if $I=I(L)$ is a GPCB ideal associated to a GPCB matrix $L$, then $\left(I, t_{1}\right)=\left(t_{1}, t_{2}^{a_{2,2}}, \ldots, t_{s}^{a_{s, s}}\right), \operatorname{rad}\left(I, t_{1}\right)=\mathfrak{m}$, and $V\left(I, t_{1}\right)=\{0\}$ (and similarly $V\left(I, t_{i}\right)=\{0\}$ for all the other variables). Moreover, by Theorem $6.3, I$ is homogeneous. Therefore, one can apply to GPCB ideals most of the results of the previous section.

In what follows, we extend to GPCB ideals some properties that hold for PCB ideals. First of all, using Theorem 6.3, we get that [30, Proposition 3.3(a)-(c)] holds for any GPCB ideal provided that we assume $s \geq 3$ in part (c). Regarding the (un)mixedness property of GPCB ideals, observe that Proposition 5.7 generalizes [30, Proposition 4.1].

To simplify notation and to avoid repetitions, for the rest of this section we assume that $L$ is a GPCB matrix with $\mathbf{b}=\left(b_{1}, \ldots, b_{s}\right) \in \mathbb{N}_{+}^{s}, \operatorname{gcd}(\mathbf{b})=1$, and $L \mathbf{b}^{\top}=0$. If $\operatorname{char}(K)=p, p$ a prime, since $\operatorname{gcd}(\mathbf{b})=1$, then on reordering the variables, one can always suppose without loss of generality that $p \nmid b_{s}$. From now on and until the end of the section, we will suppose that, if $\operatorname{char}(K)=p>0$, then $p \nmid b_{s}$. The entries of $L$ are denoted by $a_{i, i}$ and $-a_{i, j}$ if $i \neq j$. As in the proof of Theorem 6.3(c), the matrix $\widetilde{L}:=L B$, where $B:=\operatorname{diag}\left(b_{1}, \ldots, b_{s}\right)$, denotes the so-called PCB matrix associated with $L$.

Proposition 6.7 (cf. [30, Remark 3.4]). Let $I=I(L)=\left(f_{1}, \ldots, f_{s}\right)$ be the ideal of a GPCB matrix $L$ and let $\widetilde{L}$ be the PCB matrix associated with $L$. For $i=1, \ldots, s$, let $x_{i}=t_{i}^{a_{i, i}}$ and $y_{i}=t_{1}^{a_{1, i}} \cdots t_{i-1}^{a_{i-1, i}} t_{i+1}^{a_{i+1, i}} \cdots t_{s}^{a_{s, i}}$ be the two terms of $f_{i}$, so that $f_{i}=x_{i}-y_{i}$. If $b_{i}=1$, set $g_{i}=1$ and $q_{i}=0$. If $b_{i}>1$, set $g_{i}=\sum_{j=1}^{b_{i}} x_{i}^{b_{i}-j} y_{i}^{j-1} \in S$. Let $q_{i}=\sum_{j=1}^{b_{i}-1} j x_{i}^{b_{i}-1-j} y_{i}^{j-1} \in S$ and let $b(1), \ldots, b(s)$ be the vectors in $\mathbb{N}^{s}$ given by

$$
\begin{aligned}
b(1)= & \left(0,0, b_{3,3}-b_{3,4}-\cdots-b_{3, s}-b_{3,1}, b_{4,4}-b_{4,5}\right. \\
& \left.\quad-\cdots-b_{4, s}-b_{4,1}, \ldots, b_{s, s}-b_{s, 1}\right), \\
b(2)= & \left(b_{1,1}-b_{1,2}, 0,0, b_{4,4}-b_{4,5}-\cdots-b_{4, s}-b_{4,1}-b_{4,2}, \ldots, b_{s, s}-b_{s, 1}-b_{s, 2}\right), \\
b(3)= & \left(b_{1,1}-b_{1,2}-b_{1,3}, b_{2,2}-b_{2,3}, 0,0, \ldots, b_{s, s}-b_{s, 1}-b_{s, 2}-b_{s, 3}\right), \ldots, \\
b(s-1)= & \left(b_{1,1}-b_{1,2}-\cdots-b_{1, s-1}, \ldots, b_{s-2, s-2}-b_{s-2, s-1}, 0,0\right), \text { and } \\
b(s)= & \left(0, b_{2,2}-b_{2,3}-\cdots-b_{2, s}, b_{3,3}-b_{3,4}-\cdots-b_{3, s}, \ldots, b_{s-1, s-1}-b_{s-1, s}, 0\right),
\end{aligned}
$$

where $b_{i, i}$ and $-b_{i, j}, i \neq j$, are the entries of $\widetilde{L}$. Then, for each $i=1, \ldots, s$,

(a) $g_{i}$ is homogeneous and $t^{b(1)} g_{1} f_{1}+\cdots+t^{b(s)} g_{s} f_{s}=0$;

(b) $q_{i} f_{i}=g_{i}-b_{i} y_{i}^{b_{i}-1}$.

In particular, $b_{i} y_{i}^{b_{i}-1} t^{b(i)} f_{i} \in\left(f_{1}, \ldots, f_{i-1}, f_{i+1}, \ldots, f_{s}\right)+I^{2}$. 
Proof. Applying [30, Remark 3.4] to $\widetilde{L}$, the PCB matrix associated with $L$, one gets the syzygy $t^{b(1)} \widetilde{f}_{1}+\cdots+t^{b(s)} \widetilde{f}_{s}=0$, where $\widetilde{f}_{i}$ is the binomial defined by the $i$ th column of $\widetilde{L}$. Note that $\widetilde{f}_{i}=f_{\widetilde{l}_{*, i}}=x_{i}^{b_{i}}-y_{i}^{b_{i}}=\left(x_{i}-y_{i}\right)\left(x_{i}^{b_{i}-1}+\cdots+x_{i}^{b_{i}-j} y_{i}^{j}+\cdots+\right.$ $\left.y_{i}^{b_{i}-1}\right)=f_{i} g_{i}$ (even if $b_{i}=1$ ). It follows that $t^{b(1)} g_{1} f_{1}+\cdots+t^{b(s)} g_{s} f_{s}=0$. Since $\mathbf{d} L=0$ for some $\mathbf{d} \in \mathbb{N}_{+}^{s}$ (see Theorem 6.3), clearly $\mathbf{d} \widetilde{L}=0$ and $\widetilde{f}_{i}$ is homogeneous. Since $\widetilde{f}_{i}=f_{i} g_{i}$ and $S$ is a domain, $g_{i}$ is homogeneous too. This proves (a). On the other hand, one has the following identity:

$$
\begin{aligned}
q_{i} f_{i} & =\left(x_{i}^{b_{i}-2}+2 x_{i}^{b_{i}-3} y_{i}+\cdots+\left(b_{i}-1\right) y_{i}^{b_{i}-2}\right)\left(x_{i}-y_{i}\right) \\
& =x_{i}^{b_{i}-1}+x_{i}^{b_{i}-2} y_{i}+\cdots+x_{i} y_{i}^{b_{i}-2}-\left(b_{i}-1\right) y_{i}^{b_{i}-1}=g_{i}-b_{i} y_{i}^{b_{i}-1} .
\end{aligned}
$$

To see where this equality comes from, consider the polynomials $f=X-Y$ and $g=X^{b-1}+X^{b-2} Y+\cdots+Y^{b-1}$ in a polynomial ring $K[X, Y]$, where $b$ is a positive integer. Set $Z=X / Y$ and dehomogenize $f$ and $g$ to obtain $u=Z-1$ and $v=$ $Z^{b-1}+Z^{b-2}+\cdots+1$ in the Euclidean domain $K[Z]$. If $\operatorname{char}(K)=0$ or $\operatorname{char}(K)=p$, $p$ a prime with $p \nmid b, u$ and $v$ are relatively prime. The Euclidean algorithm explicitly gives us two polynomials $\alpha, \beta \in K[Z]$ with $\alpha u+\beta v=1$. On rehomogenizing and multiplying by $b$ one gets the desired identity, which holds in any characteristic. This proves (b). Finally, on multiplying the equality $q_{i} f_{i}=g_{i}-b_{i} y_{i}^{b_{i}-1}$ by $t^{b(i)} f_{i}$, one gets $b_{i} y_{i}^{b_{i}-1} t^{b(i)} f_{i}=t^{b(i)} g_{i} f_{i}-q_{i} t^{b(i)} f_{i}^{2} \in\left(f_{1}, \ldots, f_{i-1}, f_{i+1}, \ldots, f_{s}\right)+I^{2}$.

Lemma 6.8. Let $I(L)=\left(f_{1}, \ldots, f_{s}\right)$ be the ideal of a GPCB matrix L. Then, any subset of $s-1$ elements of $f_{1}, \ldots, f_{s}$ is a regular sequence in $S$.

Proof. The ideal $I(L)$ is graded by Theorem 6.3. Thus the lemma follows using the proof of [30, Proposition 3.3].

The following result generalizes [30, Proposition 3.5].

Corollary 6.9. Let $I=I(L)$ be the ideal of a GPCB matrix L. Then the following hold.

(a) For any associated prime $\mathfrak{p}$ of $I$, either $\mathrm{ht}(\mathfrak{p})=s-1$ and $t_{i} \notin \mathfrak{p}$, for all $i=1, \ldots, s$, or else $\mathfrak{p}=\mathfrak{m}$.

(b) For any minimal prime ideal $\mathfrak{p}$ over $I, I S_{\mathfrak{p}}$ is a complete intersection.

(c) If $s \geq 3, I$ is an almost complete intersection.

Proof. Item (a) follows from Remark 6.6, Theorem 6.3, and Lemma 5.1. Item (c) follows from Proposition 6.2. To prove (b), let $I=\left(f_{1}, \ldots, f_{s}\right)$, let $\mathfrak{p}$ be an arbitrary minimal prime over $I$, so $t_{i} \notin \mathfrak{p}$ for all $i=1, \ldots, s$. Either $\operatorname{char}(K)=0$, or else we may suppose that $\operatorname{char}(K)=p, p$ a prime with $p \nmid b_{s}$, because $\operatorname{gcd}(\mathbf{b})=1$ (and $s \geq 2$ ). In particular, by Proposition $6.7, b_{s} y_{s}^{b_{s}-1} \notin \mathfrak{p}$ (otherwise, if we had $b_{s} y_{s}^{b_{s}-1} \in \mathfrak{p}$, it would follow that $y_{s}$ is in $\mathfrak{p}$, so one of $t_{1}, \ldots, t_{s-1}$ is in $\mathfrak{p}$, a contradiction) and $g_{s}=q_{s} f_{s}+b_{s} y_{s}^{b_{s}-1} \notin \mathfrak{p}$. Therefore $t^{b(s)} g_{s} \notin \mathfrak{p}$ and it follows from Proposition 6.7(a) and Lemma 6.8 that $I S_{\mathfrak{p}}=\left(f_{1}, \ldots, f_{s-1}\right) S_{\mathfrak{p}}$ is generated by a regular sequence in $S_{\mathrm{p}}$.

In the next pair of results, we give an explicit description of the hull of a GPCB ideal and, if $s \geq 4$, of an irredundant embedded component.

Proposition 6.10 (cf. [30, Proposition 4.4]). Let $I=I(L)=\left(f_{1}, \ldots, f_{s}\right)$ be the ideal of a GPCB matrix L. Set $J=\left(f_{1}, \ldots, f_{s-1}\right)$. Suppose that $g \in\left(J: f_{s}\right)$ is such that $g \notin \mathfrak{p}$ for any minimal prime $\mathfrak{p}$ over $I$. Then the following hold.

(a) $I(\mathcal{L})=\operatorname{Hull}(I)=(I: g)=(J: g)$.

(b) For $b(s) \in \mathbb{N}^{s}$ and $g_{s} \in S$ as in Proposition 6.7, $I(\mathcal{L})=\left(I: t^{b(s)} g_{s}\right)=$ $\left(J: t^{b(s)} g_{s}\right)$.

Proof. Since $J$ is a graded complete intersection and $f_{s} \notin J,\left(J: f_{s}\right) \subset \mathfrak{m}$ and $g \in \mathfrak{m}$. By Proposition 5.7, $I(\mathcal{L})=\operatorname{Hull}(I)=\left(I: g^{\infty}\right)$. Hence $I \subset(J: g) \subset(I: g) \subset$ 
$\left(I: g^{\infty}\right)=I(\mathcal{L})$. To finish the proof of $($ a), just proceed as in [30, Proposition 4.4]. By Proposition 6.7, $t^{b(s)} g_{s} \in\left(J: f_{s}\right)$ and $t^{b(s)} g_{s} \in \mathfrak{m} \backslash \mathfrak{p}$, for all minimal prime ideals $\mathfrak{p}$ over $I$. Thus (b) follows from (a).

Theorem 6.11 (cf. [30, Theorem 4.10]). Let $s \geq 4$. Let $I=I(L)=\left(f_{1}, \ldots, f_{s}\right)$ be the ideal of a GPCB matrix L. Suppose that $(I: g)=\left(I: g^{\infty}\right)$ for some $g \in \mathfrak{m}, g \notin \mathfrak{p}$ for any minimal prime ideal $\mathfrak{p}$ over $I$. Then the following hold.

(a) $I+(g)$ is an irredundant $\mathfrak{m}$-primary component of $I$.

(b) For $b(s) \in \mathbb{N}^{s}$ and $g_{s} \in S$ as in Proposition $6.7, I+\left(x^{b(s)} g_{s}\right)$ is an irredundant $\mathfrak{m}$-primary component of $I$.

Proof. This is a straightforward extension of [30, Theorem 4.10].

In the light of the preceding results, and taking into account Theorem 3.3 and Proposition 5.7, it is not hard to see that analogues of [30, Theorems 6.5 and 7.1] hold for GPCB ideals. The details are left to the interested reader.

For $s=2$, we now give an explicit description of a GPCB ideal and its hull in terms of the entries of the corresponding GPCB matrix. This description will be used later in section 8 (see Proposition 8.8).

LEMMA 6.12. Let $I=I(L)=\left(f_{1}, f_{2}\right)$ be a $G P C B$ ideal associated with a $2 \times 2$ $G P C B$ matrix $L$ and let $\mathcal{L}$ be the lattice of $\mathbb{Z}^{2}$ spanned by the columns of $L$. Then there exists $\left(c_{1}, c_{2}\right) \in \mathbb{N}_{+}^{2}$ such that $I(\mathcal{L})=\left(t_{1}^{c_{1}}-t_{2}^{c_{2}}\right)$. Moreover, either $I$ is a PCB ideal, $I$ is principal, and $I=I(\mathcal{L})$ is a lattice ideal, or else $I$ is not a PCB ideal, $I$ is not principal, and $I$ is not a lattice ideal.

Proof. Set $\mathbf{b}=\left(b_{1}, b_{2}\right) \in \mathbb{N}_{+}^{2}, \operatorname{gcd}(\mathbf{b})=1$, with $L \mathbf{b}^{\top}=0$. Then $a_{1,1} b_{1}=a_{1,2} b_{2}$ and $a_{2,1} b_{1}=a_{2,2} b_{2}$. Since $\operatorname{gcd}(\mathbf{b})=1$, this forces $a_{1,1}=b_{2} c_{1}, a_{1,2}=b_{1} c_{1}$, for some $c_{1} \in \mathbb{N}_{+}$, and $a_{2,1}=b_{2} c_{2}, a_{2,2}=b_{1} c_{2}$, for some $c_{2} \in \mathbb{N}_{+}$. Therefore,

$$
L=\left(\begin{array}{rr}
a_{1,1} & -a_{1,2} \\
-a_{2,1} & a_{2,2}
\end{array}\right)=\left(\begin{array}{rr}
b_{2} c_{1} & -b_{1} c_{1} \\
-b_{2} c_{2} & b_{1} c_{2}
\end{array}\right) .
$$

Set $h:=t_{1}^{c_{1}}-t_{2}^{c_{2}}$ and $g_{i}:=t_{1}^{\left(b_{i}-1\right) c_{1}}+\cdots+t_{1}^{\left(b_{i}-j\right) c_{1}} t_{2}^{j c_{2}}+\cdots+t_{2}^{\left(b_{i}-1\right) c_{2}}$, for $i=1,2$ (if $b_{i}=1$, we understand that $g_{i}=1$ ). Then $f_{1}=t_{1}^{b_{2} c_{1}}-t_{2}^{b_{2} c_{2}}=h g_{2}$ and $f_{2}=$ $t_{2}^{b_{1} c_{2}}-t_{1}^{b_{1} c_{1}}=-h g_{1}$. Hence $I=I(L)=\left(h g_{2}, h g_{1}\right)$ and $I \subset(h)$. Since $\operatorname{gcd}(\mathbf{b})=1$, there exist $v_{1}, v_{2} \in \mathbb{Z}$ such that $1=v_{1} b_{1}+v_{2} b_{2}$. Hence

$$
v_{2}\left(a_{1,1},-a_{2,1}\right)-v_{1}\left(-a_{1,2}, a_{2,2}\right)=v_{2}\left(b_{2} c_{1},-b_{2} c_{2}\right)-v_{1}\left(-b_{1} c_{1}, b_{1} c_{2}\right)=\left(c_{1},-c_{2}\right),
$$

and $\mathcal{L}=\left\langle\left(a_{1,1},-a_{2,1}\right),\left(-a_{1,2}, a_{2,2}\right)\right\rangle=\left\langle\left(c_{1},-c_{2}\right)\right\rangle$. Therefore $I=I(L) \subset I(\mathcal{L})=$ $\left(t_{1}^{c_{1}}-t_{2}^{c_{2}}\right)=(h)$.

If $b_{1}=1$ or $b_{2}=1$, then $g_{1}=1$ or $g_{2}=1$ and $I=\left(h g_{1}, h g_{2}\right)=(h)=I(\mathcal{L})$. Moreover $I$ is the PCB ideal associated to the PCB matrix with columns $\left(c_{1},-c_{2}\right)^{\top}$ and $\left(-c_{1}, c_{2}\right)^{\top}$.

Suppose that $b_{1}, b_{2}>1$ with $\operatorname{gcd}(\mathbf{b})=1$. Then $b_{1} \nmid b_{2}$ and $b_{2} \nmid b_{1}$. It follows that $f_{2} \nmid f_{1}$ and $f_{1} \nmid f_{2}$ (see, e.g., [29, Lemma 8.2]). Since $I=\left(f_{1}, f_{2}\right)$ is homogeneous, $f_{1}, f_{2}$ is a minimal homogeneous system of generators of $I$. Hence $I$ is not principal and $h \in I(\mathcal{L}) \backslash I$. In particular, $I$ is not a PCB ideal (by [30, Remark 2.3]) and is not a lattice ideal (see Proposition 5.7(b)).

We finish the section with an example.

Example 6.13. Let $L$ be the following PCB matrix and let $I=I(L)$ and $I^{\top}=$ $I\left(L^{\top}\right)$ be the matrix ideals of $L$ and $L^{\top}$, respectively:

$$
L=\left(\begin{array}{rrrr}
4 & -2 & -1 & -1 \\
-1 & 4 & -2 & -1 \\
-1 & -1 & 3 & -1 \\
-1 & -1 & -1 & 3
\end{array}\right)
$$

Copyright $@$ by SIAM. Unauthorized reproduction of this article is prohibited. 
Observe that $L \mathbf{1}^{\top}=0$ and $L^{\top} \mathbf{b}^{\top}=0$ with $\mathbf{b}=(20,24,31,25)$ and $\mathbf{1}=(1,1,1,1)$. Let $\mathcal{L}$ and $\mathcal{L}^{\top}$ be the lattices spanned by the columns of $L$ and $L^{\top}$, respectively. Here $\Delta_{3}(L)=1$. By Corollary $5.9, \operatorname{deg}(S / I)=31$ and $\operatorname{deg}\left(S / I^{\top}\right)=1$. Moreover $I=\mathfrak{p}_{\mathbf{b}} \cap \mathfrak{q}$ and $I^{\top}=\mathfrak{p}_{1} \cap \mathfrak{q}^{\prime}$ are minimal primary decompositions of $I$ and $I^{\top}$, respectively, where $I(\mathcal{L})=\mathfrak{p}_{\mathbf{b}}$ is the Herzog ideal associated with $\mathbf{b}, I\left(\mathcal{L}^{\top}\right)=\mathfrak{p}_{\mathbf{1}}$ is the Herzog ideal associated with $\mathbf{1}$, and $\mathfrak{q}$ and $\mathfrak{q}^{\prime}$ are $\mathfrak{m}$-primary ideals which can be calculated explicitly (see Theorem 6.11).

7. Laplacian matrices and ideals. In this section we show how our results can be applied to an interesting family of binomial ideals arising from Laplacian matrices.

Connecting combinatorial properties of graphs to linear-algebraic properties of Laplacian matrices has attracted a great deal of attention $[4,14,26]$. We are interested in relating the combinatorics of the graph with the algebraic invariants and properties of the binomial ideals associated with Laplacian matrices.

Let $S=K\left[t_{1}, \ldots, t_{s}\right]$ be a polynomial ring over a field $K$ and let $G=(V, E, w)$ be a weighted connected simple graph, where $V=\left\{t_{1}, \ldots, t_{s}\right\}$ is the set of vertices, $E$ is the set of edges, and $w$ is a weight function that associates a weight $w_{e}$ with every edge $e$ in the graph. Edges of $G$ are unordered pairs $\left\{t_{i}, t_{j}\right\}$ with $i \neq j$. To define the Laplacian matrix, recall that the adjacency matrix $A(G)$ of this graph is given by

$$
A(G)_{i, j}:= \begin{cases}w_{e} & \text { if } e=\left\{t_{i}, t_{j}\right\} \in E \\ 0 & \text { otherwise }\end{cases}
$$

Now, the Laplacian $L(G)$ of the graph $G$ is defined as $L(G):=D(G)-A(G)$, where $D(G)$ is a diagonal matrix with entry $D(G)_{i, i}$ equal to the weighted degree $\sum_{e \in E\left(t_{i}\right)} w_{e}$ of the vertex $t_{i}$. Here, we denoted by $E\left(t_{i}\right)$ the set of edges adjacent (incident) to $t_{i}$. One can check that the entries of the Laplacian are given by

$$
L(G)_{i, j}:= \begin{cases}\sum_{e \in E\left(t_{i}\right)} w_{e} & \text { if } i=j \\ -w_{e} & \text { if } i \neq j \text { and } e=\left\{t_{i}, t_{j}\right\} \in E \\ 0 & \text { otherwise }\end{cases}
$$

Notice that $L(G)$ is symmetric and $\mathbf{1}(G)=0$. The Laplacian matrix is a prime example of a CB matrix. The Laplacian matrices of complete graphs are PCB matrices; this type of matrix occurs in [23]. The binomial ideal $I \subset S$ defined by the columns of $L(G)$ is called the Laplacian ideal of $G$. If $I \subset S$ is the Laplacian ideal of $G$, the lattice ideal $I(\mathcal{L})=\left(I:\left(t_{1} \cdots t_{s}\right)^{\infty}\right)$ is called the toppling ideal of the graph [23, 32]. If $G$ is connected, the toppling ideal is a lattice ideal of dimension 1.

The torsion subgroup of the factor group $\mathbb{Z}^{s} / \operatorname{Im}(L(G))$, denoted by $K(G)$, is called the critical group or the sandpile group of $G$ (see [1,22] for additional information). Notice that $K(G)$ is the torsion subgroup of $\mathbb{Z}^{s} / \mathcal{L}$. The structure, as a finite abelian group, of $K(G)$ is only known for a few families of graphs (see [1] and the references therein). If $G$ is regarded as a multigraph (where each edge $e$ occurs $w_{e}$ times), then by Kirchhoff's matrix tree theorem, the order of $K(G)$ is the number of spanning trees of $G$ and this number is equal to the $(i, j)$-entry of the adjoint matrix of $L(G)$ for any $(i, j)$ (see [4, Theorem 6.3, p. 39] and [26, Theorem 1.1]).

Next we give an application of our earlier results to this setting.

Proposition 7.1. Let $G=(V, E, w)$ be a connected weighted simple graph with vertices $t_{1}, \ldots, t_{s}$ and let $I \subset S$ be its Laplacian ideal. Then the following hold.

(a) $V\left(I, t_{i}\right)=\{0\}$ for all $i$.

(b) $\operatorname{deg}(S / I)=\operatorname{deg}(S / I(\mathcal{L}))=|K(G)|$. 


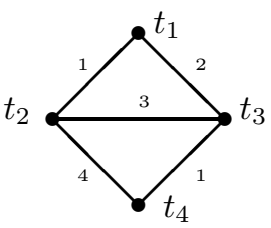

$$
L(G)=\left(\begin{array}{rrrr}
3 & -1 & -2 & 0 \\
-1 & 8 & -3 & -4 \\
-2 & -3 & 6 & -1 \\
0 & -4 & -1 & 5
\end{array}\right)
$$

FIG. 1. Weighted graph $G$.

(c) $\operatorname{Hull}(I)=I(\mathcal{L})$.

(d) If $\left|E\left(t_{i}\right)\right| \geq 3$ for all $i$, then $I$ is not a lattice ideal.

(e) If $G=\mathcal{K}_{s}$ is a complete graph, then $\operatorname{deg}(S / I)=s^{s-2}$.

(f) If $G$ is a tree, then $\operatorname{deg}(S / I)=\operatorname{deg}(S / I(\mathcal{L}))=1$.

Proof. (a) For $1 \leq k \leq s$, let $f_{k}$ be the binomial defined by the $k$ th column of $L(G)$. Fix $i$ such that $1 \leq i \leq s$. Clearly $\{0\}$ is contained in $V\left(I, t_{i}\right)$ because $I$ is graded. To show the reverse containment, let $\alpha=\left(\alpha_{1}, \ldots, \alpha_{s}\right)$ be a point in $V\left(I, t_{i}\right)$. If $\left\{t_{i}, t_{k}\right\} \in E\left(t_{i}\right)$, we claim that $\alpha_{k}=0$. We can write

$$
f_{k}=t_{k}^{\sum_{e \in E\left(t_{k}\right)} w_{e}}-\prod_{e \in E\left(t_{k}\right), t_{j} \in e} t_{j}^{w_{e}} .
$$

Using that $f_{k}(\alpha)=0$, we get $\alpha_{k}^{\sum_{e \in E\left(t_{k}\right)} w_{e}}=\prod_{\left\{e \in E\left(t_{k}\right), t_{j} \in e\right\}} \alpha_{j}^{w_{e}}$. Since $\left\{t_{i}, t_{k}\right\}$ is in $E\left(t_{k}\right)$ and using that $\alpha_{i}=0$, we obtain that $\alpha_{k}=0$, as claimed. Let $\ell$ be an integer in $\{1, \ldots, s\}$. Since the graph $G$ is connected, there is a path $\left\{v_{1}, \ldots, v_{r}\right\}$ joining $t_{i}$ and $t_{\ell}$, i.e., $v_{1}=t_{i}, v_{r}=t_{\ell}$, and $\left\{v_{j}, v_{j+1}\right\} \in E(G)$ for all $j$. There is a permutation $\pi$ of $V$ such that $\pi(1)=i, \pi(r)=\ell$, and $v_{j}=t_{\pi(j)}$ for $j=1, \ldots, r$. Then $t_{\pi(j)} \in E\left(t_{\pi(j-1)}\right)$ for $j=2, \ldots, r$. Applying the claim successively for $j=2, \ldots, r$, we obtain $\alpha_{\pi(2)}=0, \alpha_{\pi(3)}=0, \ldots, \alpha_{\pi(r)}=0$. Thus, $\alpha_{\ell}=0$. This proves that $\alpha=0$.

(b) and (c) These two parts follow from Proposition 2.5, Corollary 4.9, and Proposition $5.7(\mathrm{a})$, since $L(G)$ is homogeneous with respect to the weight vector $\mathbf{1}$.

(d) This part follows from Proposition 6.1(a). $s^{s-2}$.

(e) By part (b) one has $\operatorname{deg}(S / I)=|K(G)|$, and by [4, p. 39] one has $|K(G)|=$

(f) Since $|K(G)|$ is the number of spanning trees of the graph $G$, this number is equal to 1 . Thus, $K(G)=\{0\}$, i.e., $K(G)$ is torsion free. By (b), we get that $\operatorname{deg}(S / I)=1$.

Example 7.2. Let $G$ be the weighted graph of Figure 1 and let $I$ be its Laplacian ideal. Then $I=\left(t_{1}^{3}-t_{2} t_{3}^{2}, t_{2}^{8}-t_{1} t_{3}^{3} t_{4}^{4}, t_{3}^{6}-t_{1}^{2} t_{2}^{3} t_{4}, t_{4}^{5}-t_{2}^{4} t_{3}\right), \operatorname{deg}(S / I)=67$, and

$\operatorname{Hull}(I)=I(\mathcal{L})=\left(t_{1}^{3}-t_{2} t_{3}^{2}, t_{1} t_{3}^{4}-t_{2}^{4} t_{4}, t_{2}^{4} t_{3}-t_{4}^{5}, t_{3}^{6}-t_{1}^{2} t_{2}^{3} t_{4}, t_{2}^{8}-t_{1} t_{3}^{3} t_{4}^{4}, t_{1}^{2} t_{2}^{7}-t_{3}^{5} t_{4}^{4}\right)$.

If $K=\mathbb{Q}$, the toppling ideal $I(\mathcal{L})$ has two primary components of degrees 66 and 1 .

As another application, by Proposition 6.2, the Laplacian ideal is an almost complete intersection for any connected simple graph without vertices of degree 1 .

Proposition 7.3. Let $G=(V, E, w)$ be a connected weighted simple graph with vertices $t_{1}, \ldots, t_{s}$ and let $I \subset S$ be its Laplacian ideal. If $\left|E\left(t_{i}\right)\right| \geq 2$ for all $i$, then $I$ is an almost complete intersection.

The notion of a Laplacian matrix can be extended to weighted digraphs; see [8] and the references therein. Let $G=(V, E, w)$ be a weighted digraph without loops and with vertices $t_{1}, \ldots, t_{s}$, let $w\left(t_{i}, t_{j}\right)$ be the weight of the directed arc from $t_{i}$ to $t_{j}$, and let $A(G)$ be the adjacency matrix of $G$ given by $A(G)_{i, j}=w\left(t_{i}, t_{j}\right)$. The Laplacian matrix of $G$ is given by $L(G)=D^{+}(G)-A(G)$, where $D^{+}(G)$ is the diagonal matrix with the out-degrees of the vertices of $G$ in the diagonal entries. Note that 
$L(G) \mathbf{1}^{\top}=0$ and that the Laplacian matrix of a digraph may not be symmetric (see Example 7.8). If $t_{i}$ is a sink, i.e., there is no arc of the form $\left(t_{i}, t_{j}\right)$, then the $i$ th row of $L(G)$ is zero. Thus, the rank of $L(G)$ may be much less than $s-1$. When $G$ is a strongly connected digraph, it is well-known that the rank of $L(G)$ is $s-1$. This follows from the Perron-Frobenius theorem; see the proof of Theorem 7.5 .

Let $G$ be a weighted digraph without sources or sinks, i.e., for each vertex $t_{i}$ there is at least one arc of the form $\left(t_{j}, t_{i}\right)$ and one arc of the form $\left(t_{i}, t_{k}\right)$. Then $L(G)$ is a $\mathrm{CB}$ matrix. Conversely if $L$ is a $\mathrm{CB}$ matrix as in Definition 1.1, then $L$ is the Laplacian matrix of the weighted digraph $G$ defined as follows. A pair $\left(t_{i}, t_{j}\right)$ is an arc of $G$ if and only if $i \neq j$ and $a_{i, j} \neq 0$. The weight of the arc $\left(t_{i}, t_{j}\right)$ is $a_{i, j}$.

Definition 7.4 (see [14, pp. 175 and 29]). Let $A=\left(a_{i, j}\right)$ be an $s \times s$ real matrix. The underlying digraph of $A$, denoted by $G_{A}$, has vertex set $\left\{t_{1}, \ldots, t_{s}\right\}$ with an arc from vertex $t_{i}$ to vertex $t_{j}$ if and only if $a_{i, j} \neq 0$. Note that this digraph may have loops. A digraph is called strongly connected if any two vertices can be joined by a directed path.

The underlying digraph of the matrix of Example 7.2 is strongly connected. If $G$ is a weighted digraph and $L$ is its Laplacian matrix, then $G$ is obtained from the underlying digraph $G_{L}$ of $L$ by removing all loops of $G_{L}$.

THEOREM 7.5. Let $L$ be a GCB matrix and let $G$ be its underlying graph. If $G$ is strongly connected, then $\operatorname{rank}(L)=s-1$ and $L^{\top}$ is a GCB matrix.

Proof. By passing to the associated $\mathrm{CB}$ matrix $\widetilde{L}$, we may assume that $L$ is a $\mathrm{CB}$ matrix (see the proof of Theorem 6.3(c)). Let $L$ be a CB matrix as in Definition 1.1. We can write $L=D-A$, where $D=\operatorname{diag}\left(a_{1,1}, \ldots, a_{s, s}\right)$ and $A$ is the matrix whose $i, j$ entry is $a_{i, j}$ if $i \neq j$ and whose diagonal entries are equal to zero. We set $\delta_{i}=a_{i, i}$ for $i=1, \ldots, s$. By hypothesis $L \mathbf{1}^{\top}=0$, hence $\operatorname{rank}(L) \leq s-1$. There exists a nonzero vector $\mathbf{b} \in \mathbb{Z}^{s}$ such that $\mathbf{b} L=0$. Therefore

$$
\mathbf{b} D=\mathbf{b} A=\mathbf{b} D\left(D^{-1} A\right) .
$$

Since $L \mathbf{1}^{\top}=0$, we get that $D \mathbf{1}^{\top}=A \mathbf{1}^{\top}$ or, equivalently, $\left(D^{-1} A\right) \mathbf{1}^{\top}=\mathbf{1}^{\top}$. Thus, as the entries of $D^{-1} A$ are nonnegative, the matrix $B:=D^{-1} A$ is stochastic. It is well known that the spectral radius $\rho(B)$ of a stochastic matrix $B$ is equal to $1[3$, Theorem 5.3], where $\rho(B)$ is the maximum of the moduli of the eigenvalues of $B$. As the diagonal entries of $B$ are zero and $\delta_{i}>0$ for all $i$, the underlying digraph $G_{B}$ of $B$ is equal to the digraph obtained from $G$ by removing all loops of $G$. Since $G$ is strongly connected, so is $G_{B}$, and by the Perron-Frobenius theorem for nonnegative matrices [14, Theorem 8.8.1, p. 178], $\rho(B)=1$ and 1 is a simple eigenvalue of $B$ (i.e., the eigenspace of $B$ relative to $\rho(B)=1$ is 1 dimensional), and if $z$ is an eigenvector for $\rho(B)=1$, then no entries of $z$ are zero and all have the same sign. Applying this to $z=\mathbf{b} D$, we get that $b_{i} \neq 0$ for all $i$ and all entries of $\mathbf{b}$ have the same sign. Hence, $\operatorname{ker}\left(L^{\top}\right)=\left(\mathbf{b}^{\top}\right)$ for any nonzero vector $\mathbf{b}$ such that $\mathbf{b} L=0$, so $L^{\top}$ is a GCB matrix of rank $s-1$.

The results of the previous sections can also be applied to GCB ideals that arise from matrices with strongly connected underlying digraphs.

Proposition 7.6. Let $L$ be a GCB matrix of size $s \times s$, let $G_{L}$ be the underlying digraph of $L$, and let $I=I\left(L^{\top}\right)$ be the matrix ideal of $L^{\top}$. The following conditions are equivalent.

(a) $G_{L}$ is strongly connected.

(b) $V\left(I, t_{i}\right)=\{0\}$ for all $i$.

(c) $L_{i, j}>0$ for all $i, j$, where $\operatorname{adj}(L)=\left(L_{i, j}\right)$ is the adjoint of $L$. 


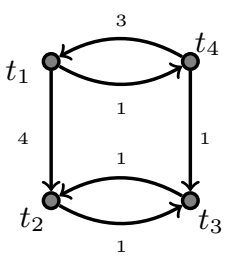

$$
L=L(G)=\left(\begin{array}{rrrr}
5 & -4 & 0 & -1 \\
0 & 1 & -1 & 0 \\
0 & -1 & 1 & 0 \\
-3 & 0 & -1 & 4
\end{array}\right)
$$

FiG. 2. A weighted digraph $G$ with four vertices and its Laplacian matrix.

Proof. By passing to the associated matrix $\widetilde{L}$, we may assume that $L$ is a $\mathrm{CB}$ matrix (see the proof of Theorem 6.3(c)).

(a) $\Rightarrow$ (b). Since any two vertices can be joined by a directed path, the proof follows adapting the argument given to prove Proposition 7.1(a).

(b) $\Rightarrow$ (a). We proceed by contradiction. Assume that $G_{L}$ is not strongly connected. Without loss of generality we may assume that there is no directed path from $t_{1}$ to $t_{s}$. Let $W$ be the set of all vertices $t_{i}$ such that there is a directed path from $t_{i}$ to $t_{s}$, the vertex $t_{s}$ being included in $W$. The set $W$ is nonempty because $G_{L}$ has no sources or sinks by the definition of a CB matrix, and the vertex $t_{1}$ is not in $W$. Consider the vector $\alpha \in K^{s}$ defined as $\alpha_{i}=1$ if $t_{i} \notin W$ and $\alpha_{i}=0$ if $t_{i} \in W$. To derive a contradiction it suffices to show that all binomials of $I\left(L^{\top}\right)$ vanish at the nonzero vector $\alpha$. Let $L$ be as in Definition 1.1 and let $f_{k}=t_{k}^{a_{k, k}}-\prod_{j \neq k} t_{j}^{a_{k, j}}$ be the binomial defined by the $k$-row of $L$. If $t_{k} \in W$, there is a directed path $\mathcal{P}$ from $t_{k}$ to $t_{s}$. Then $t_{j}$ is part of the path $\mathcal{P}$ for some $j$ such that $a_{k, j}>0$. Thus, since $t_{j} \in W$, $f_{k}(\alpha)=0$. If $t_{k} \notin W$, then $t_{j}$ is not in $W$ for any $j$ such that $a_{k, j}>0$, because if $a_{k, j}>0$, the pair $\left(t_{k}, t_{j}\right)$ is an arc of $G_{L}$. Thus, $f_{k}(\alpha)=0$.

(a) $\Rightarrow$ (c). By the proof of Theorem 6.3(c), one has that $L_{i, i} \geq 0$ for all $i$. By Theorem 7.5 and using the proof of Theorem 6.3(a), we get that $L_{i, j}>0$ for all $i, j$.

(c) $\Rightarrow$ (a). We proceed by contradiction. Assume that $G_{L}$ is not strongly connected. We may assume that there is no directed path from $t_{1}$ to $t_{s}$. Let $W$ be as above and let $W^{c}=\left\{t_{i} \mid t_{i} \notin W\right\}$ be its complement. We can write $W^{c}=\left\{t_{\ell_{1}}, \ldots, t_{\ell_{r}}\right\}$. Consider the $r \times r$ submatrix $B$ obtained from $L$ by fixing rows $\ell_{1}, \ldots, \ell_{r}$ and columns $\ell_{1}, \ldots, \ell_{r}$. Notice that by the arguments above $W^{c}=\cup_{t_{k} \in W^{c}}\left(\operatorname{supp}\left(f_{k}\right)\right)$. Hence any row of $B$ extends to a row of $L$ by adding 0's only, and, consequently, the sum of the columns of $B$ is zero. Hence $\operatorname{det}(B)=0$. By permuting rows and columns, $L$ can be brought to the form

$$
L^{\prime}=\left(\begin{array}{cc}
C & 0 \\
C^{\prime} & C^{\prime \prime}
\end{array}\right)
$$

where $C$ and $C^{\prime \prime}$ are square matrices of orders $r$ and $s-r$, respectively, and $\operatorname{det}(C)=0$. Hence the adjoint of $L^{\prime}$ has a zero entry, and so does the adjoint of $L$, a contradiction.

Corollary 7.7. Let $L$ be a GCB matrix of size $s \times s$ and let $\left(L_{i, j}\right)$ be its adjoint. If $G_{L}$ is strongly connected, then

$$
\operatorname{gcd}\left(\left\{L_{i, k}\right\}_{i=1}^{s}\right) \operatorname{deg}\left(S / I\left(L^{\top}\right)\right)=\max \left(\left\{L_{i, k}\right\}_{i=1}^{s}\right) \operatorname{gcd}\left(\left\{L_{i, j}\right\}\right) \text { for any } k .
$$

Proof. By Proposition 7.6, all entries of $\operatorname{adj}(L)$ are positive and any column of $\operatorname{adj}(L)$ gives a grading for $I\left(L^{\top}\right)$. Hence the formula follows from Corollary 5.9.

Example 7.8. Let $G$ be the weighted digraph of Figure 2 and let $L$ be its Laplacian matrix. The digraph $G_{L}$ is not strongly connected, the CB ideal $I\left(L^{\top}\right)$ is graded but $I(L)$ is not. 
8. Homogeneous lattice ideals of dimension 1 in 3 variables. The main results of this section uncover the structure of lattice ideals of dimension 1 in 3 variables and the structure of the homogeneous lattices of rank 2 in $\mathbb{Z}^{3}$.

Definition 8.1. Let $I$ be a binomial ideal of $S$ and let $f=t^{a}-t^{b} \in I$. We will say that

(a) $f$ is $t_{i}$-pure if $t^{a}$ and $t^{b}$ are nonconstant, have no common variables, and $\operatorname{supp}(a)=\{i\}$

(b) $f$ is $t_{i}$-critical if $f$ is $t_{i}$-pure and for any other $t_{i}$-pure binomial $g=t_{i}^{c_{i}}-t^{d}$ of $I, c_{i} \geq a_{i}$

(c) a full set of pure (respectively, critical) binomials of $I$ is a family $f_{1}, \ldots, f_{s}$ of binomials where each $f_{i}$ is a $t_{i}$-pure (respectively, $t_{i}$-critical) binomial of $I$.

We begin with a result that complements the well-known result of Herzog [16] that shows that the toric ideal of a monomial space curve is generated by a full set of critical binomials.

TheOREM 8.2. Let $S=K\left[t_{1}, t_{2}, t_{3}\right]$ and let $I$ be a homogeneous lattice ideal of $S$ of height 2. Then $I$ is generated by a full set of critical binomials. Concretely, and with a suitable renumbering of the variables, only the two following cases can occur:

(a) $I$ is minimally generated by $f_{1}=t_{1}^{a_{1}}-t_{3}^{c_{3}}$ and $f_{2}=t_{2}^{b_{2}}-t_{1}^{b_{1}} t_{3}^{b_{3}}$ with $0 \leq b_{1} \leq$ $a_{1}, a_{1}, b_{2}, c_{3}>0$, and $b_{1}+b_{3}>0$;

(b) $I$ is minimally generated by $f_{1}=t_{1}^{a_{1}}-t_{2}^{a_{2}} t_{3}^{a_{3}}, f_{2}=t_{2}^{b_{2}}-t_{1}^{b_{1}} t_{3}^{b_{3}}$, and $f_{3}=$ $t_{3}^{c_{3}}-t_{1}^{c_{1}} t_{2}^{c_{2}}$ with $0<a_{2}<b_{2}, 0<a_{3}<c_{3}, 0<b_{1}<a_{1}, 0<b_{3}<c_{3}$, $0<c_{1}<a_{1}$, and $0<c_{2}<b_{2}$. Moreover, $a_{1}=b_{1}+c_{1}, b_{2}=a_{2}+c_{2}$, and $c_{3}=a_{3}+b_{3}$.

Proof. Let $\mathbf{d}=\left(d_{1}, d_{2}, d_{3}\right), \operatorname{gcd}(\mathbf{d})=1$, be the grading in $S$ under which $I$ is homogeneous. Since $I$ is a lattice ideal, $t_{i}$ is a nonzero divisor of $S / I$ for $i=1,2,3$. In particular, $I$ can be generated by pure binomials, i.e., binomials of the form $t_{1}^{e_{1}}-t_{2}^{e_{2}} t_{3}^{e_{3}}$ with $e_{1}>0$, and similarly for $i=2,3$.

Since $I$ is a homogeneous lattice ideal of height 2, by [20, Proposition 2.9], $V\left(I, t_{i}\right)=\{0\}$ for all $i$. In particular, $I$ contains $t_{i}$-pure binomials, for $i=1,2,3$. Indeed, if $I$ contains no $t_{3}$-pure binomials, say, then $V\left(I, t_{1}\right) \supset V\left(t_{1}, t_{2}\right) \neq\{0\}$, a contradiction.

Therefore there exist $f_{1}=t_{1}^{a_{1}}-t_{2}^{a_{2}} t_{3}^{a_{3}}, f_{2}=t_{2}^{b_{2}}-t_{1}^{b_{1}} t_{3}^{b_{3}}$, and $f_{3}=t_{3}^{c_{3}}-t_{1}^{c_{1}} t_{2}^{c_{2}}$, a full set of critical binomials of $I$; i.e., $a_{1}, b_{2}, c_{3}>0$ and for any $t_{1}$-pure binomial of $I$ of the form $t_{1}^{e_{1}}-t_{2}^{e_{2}} t_{3}^{e_{3}}$, one has $e_{1} \geq a_{1}$, and similarly with the other variables $t_{2}$ and $t_{3}$. Notice that one could have $f_{j}=-f_{i}$ for $j \neq i$.

Following the proof of Kunz in [19, pp. 137-140], one can show that $I$ is generated by a full set of critical binomials. For the sake of clarity, we outline the main details of the proof.

After renumbering the variables one may suppose that $f_{1}$ is the one of least degree among $f_{1}, f_{2}$, and $f_{3}$. Then $a_{2} \leq b_{2}$ and $a_{3} \leq c_{3}$. Moreover, $a_{2}=b_{2}$ is equivalent to $a_{3}=0$, and, in this case, $-f_{1}=t_{2}^{b_{2}}-t_{1}^{a_{1}}$ is $t_{2}$-critical and one may choose $f_{2}$ to be $-f_{1}$. Similarly, $a_{3}=c_{3}$ is equivalent to $a_{2}=0$, and, in this case, $-f_{1}=t_{3}^{c_{3}}-t_{1}^{a_{1}}$ is $t_{3}$-critical and one may choose $f_{3}$ to be $-f_{1}$.

If $a_{2}=b_{2}$, (i.e., $a_{3}=0$ ), we may interchange the numbering of the variables $t_{2}$ and $t_{3}$ so that, in the new numbering, $a_{3}=c_{3}$ and $a_{2}=0$. Hence, there are only the following two cases for $f_{1}$ :

(a) $f_{1}=t_{1}^{a_{1}}-t_{3}^{c_{3}}$, if $a_{2}=0$ and $a_{3}=c_{3}$;

(b) $f_{1}=t_{1}^{a_{1}}-t_{2}^{a_{2}} t_{3}^{a_{3}}$ with $0<a_{2}<b_{2}$ and $0<a_{3}<c_{3}$.

CASE (a). Here $f_{1}=t_{1}^{a_{1}}-t_{3}^{c_{3}}, f_{2}=t_{2}^{b_{2}}-t_{1}^{b_{1}} t_{3}^{b_{3}}$, and $f_{3}=t_{3}^{c_{3}}-t_{1}^{a_{1}}=-f_{1}$ with $\operatorname{deg}\left(f_{1}\right) \leq \operatorname{deg}\left(f_{2}\right)$. Moreover, one can suppose that $0 \leq b_{1} \leq a_{1}$. 
In a rather long, but not difficult way, one proves that for any pure binomial $f$ of $I$, either $f$ is in $\left(f_{1}, f_{2}\right)$ or $f$ modulo $\left(f_{1}, f_{2}\right)$ is a multiple of a binomial $g$ of $I$ with $\operatorname{deg}(g)<\operatorname{deg}(f)$. One starts by taking $f=t_{1}^{e_{1}}-t_{2}^{e_{2}} t_{3}^{e_{3}} \in I$ with $e_{1} \geq a_{1}$, and proving that $f-t_{1}^{e_{1}-a_{1}} f_{1}$ is a multiple of a binomial $g \in I$ with $\operatorname{deg}(g)<\operatorname{deg}(f)$. There is an analogous argument if $f=t_{2}^{e_{2}}-t_{1}^{e_{1}} t_{3}^{e_{3}} \in I$ or $f=t_{3}^{e_{3}}-t_{1}^{e_{1}} t_{2}^{e_{2}} \in I$.

One concludes that $I=\left(f_{1}, f_{2}\right)$. Indeed, suppose not and take $f$ a pure binomial in $I \backslash\left(f_{1}, f_{2}\right)$ of the smallest possible degree. We have seen that there exists $h \in\left(f_{1}, f_{2}\right)$ such that $f-h$ is a multiple of a binomial $g \in I$ with $\operatorname{deg}(g)<\operatorname{deg}(f)$. Since $f$ is the element in $I \backslash\left(f_{1}, f_{2}\right)$ of the smallest possible degree, this forces $g$ to be in $\left(f_{1}, f_{2}\right)$. Hence $f \in(g, h) \subset\left(f_{1}, f_{2}\right)$, a contradiction.

CASE (b). Here $f_{1}=t_{1}^{a_{1}}-t_{2}^{a_{2}} t_{3}^{a_{3}}, f_{2}=t_{2}^{b_{2}}-t_{1}^{b_{1}} t_{3}^{b_{3}}$ and $f_{3}=t_{3}^{c_{3}}-t_{1}^{c_{1}} t_{2}^{c_{2}}$ with $0<a_{2}<b_{2}$ and $0<a_{3}<c_{3}$.

Possibly after renumbering $t_{2}$ and $t_{3}$, one can assume that the degree of $f_{2}$ is smaller than the degree of $f_{3}$. Observe that $b_{1}<a_{1}$ and, in particular, $b_{3}>0$. Analogously, $c_{1}<a_{1}$ and, in particular, $c_{2}>0$. Moreover $b_{3} \leq c_{3}$, and $b_{3}=c_{3}$ is equivalent to $b_{1}=0$. In this case, $-f_{2}=t_{3}^{c_{3}}-t_{2}^{b_{2}}$ is $t_{3}$-critical and one may choose as $f_{3}$ the binomial $-f_{2}$.

Therefore, there are only the following two cases for $f_{2}$ :

(b1) $f_{2}=t_{2}^{b_{2}}-t_{3}^{c_{3}}$, if $b_{1}=0$ and $b_{3}=c_{3}$;

(b2) $f_{2}=t_{2}^{b_{2}}-t_{1}^{b_{1}} t_{3}^{b_{3}}$ with $0<b_{1}<a_{1}$ and $0<b_{3}<c_{3}$.

CASE (b1). Here $f_{1}=t_{1}^{a_{1}}-t_{2}^{a_{2}} t_{3}^{a_{3}}, f_{2}=t_{2}^{b_{2}}-t_{3}^{c_{3}}$ and $f_{3}=t_{3}^{c_{3}}-t_{2}^{b_{2}}=-f_{2}$ with $0<a_{2}<b_{2}, 0<a_{3}<c_{3}$, and $\operatorname{deg}\left(f_{1}\right) \leq \operatorname{deg}\left(f_{2}\right)$. Similarly to the proof of Case (a), one can show that $I=\left(f_{1}, f_{2}\right)$ (although now the doubly pure binomial has a greater degree than the other binomial).

CASE (b2). Here $f_{1}=t_{1}^{a_{1}}-t_{2}^{a_{2}} t_{3}^{a_{3}}, f_{2}=t_{2}^{b_{2}}-t_{1}^{b_{1}} t_{3}^{b_{3}}$, and $f_{3}=t_{3}^{c_{3}}-t_{1}^{c_{1}} t_{2}^{c_{2}}$ with $0<a_{2}<b_{2}, 0<a_{3}<c_{3}, 0<b_{1}<a_{1}$, and $0<b_{3}<c_{3}$. Moreover, $0 \leq c_{1}<a_{1}$, $c_{2}>0$, and $\operatorname{deg}\left(f_{1}\right) \leq \operatorname{deg}\left(f_{2}\right) \leq \operatorname{deg}\left(f_{3}\right)$. Since $b_{3}>0$, then $c_{2}<b_{2}$. Since $b_{3}<c_{3}$ (and $b_{1} \neq 0$ ), then $c_{1}>0$.

As in Case (a), one can prove that, for each pure binomial $f$ of $I$, either $f$ is in $\left(f_{1}, f_{2}, f_{3}\right)$ or $f$ modulo $\left(f_{1}, f_{2}, f_{3}\right)$ is a multiple of a binomial $g$ of $I$ with $\operatorname{deg}(g)<$ $\operatorname{deg}(f)$. One concludes, as before, that $I=\left(f_{1}, f_{2}, f_{3}\right)$.

In Case (b2), $I$ is minimally generated by $f_{1}, f_{2}$, and $f_{3}$. Indeed, if $f_{3} \in\left(f_{1}, f_{2}\right)$, say, then on taking $t_{1}=0$ and $t_{2}=0$, one would get a contradiction.

Finally, $a_{1}=b_{1}+c_{1}, b_{2}=a_{2}+c_{2}$, and $c_{3}=a_{3}+b_{3}$ (see [19, p. 139, line 15]). Indeed, let $\alpha_{1}:=a_{1}-b_{1}-c_{1}, \alpha_{2}:=b_{2}-a_{2}-c_{2}$, and $\alpha_{3}:=c_{3}-a_{3}-b_{3}$. Clearly $\alpha_{1} d_{1}+$ $\alpha_{2} d_{2}+\alpha_{3} d_{3}=0$. We may suppose that $\alpha_{2}$ and $\alpha_{3}$, say, have the same sign. Then necessarily $\alpha_{1}=0$, because if not, since $f_{1}$ is $t_{1}$-critical and either $t_{1}^{\alpha_{1}}-t_{2}^{-\alpha_{2}} t_{3}^{-\alpha_{3}} \in I$ or $t_{1}^{-\alpha_{1}}-t_{2}^{\alpha_{2}} t_{3}^{\alpha_{3}} \in I$, we get that either $\alpha_{1} \geq a_{1}$ or $-\alpha_{1} \geq a_{1}$, which would imply that either $-b_{1}-c_{1} \geq 0$ or $b_{1}+c_{1} \geq 2 a_{1}$, in contradiction to $0<b_{1}<a_{1}$ and $0<c_{1}<a_{1}$. Thus $\alpha_{1}=0$, so $\alpha_{2}=0$ and $\alpha_{3}=0$.

Corollary 8.3. If $I$ is a lattice ideal of dimension 0 and $s=2$, then $I$ is generated by at most 3 binomials.

Proof. By Lemma 4.1, $I^{h} \subset S[u]$ is a graded lattice ideal of dimension 1. Thus, the result follows from Theorem 8.2.

Before proceeding with the main result of the section, we state some properties of GCB and CB binomial ideals in the case $s=3$.

Lemma 8.4. Let $I=I(L)$ be the GCB ideal associated with $a 3 \times 3$ GCB matrix $L$. Then $I$ is homogeneous and $V\left(I, t_{i}\right)=\{0\}$ for all $i$. 
Proof. Let $L$ be a $3 \times 3$ GCB matrix, where $\mathbf{b}=\left(b_{1}, b_{2}, b_{3}\right) \in \mathbb{N}_{+}^{3}, \operatorname{gcd}(\mathbf{b})=1$, and $L \mathbf{b}^{\top}=0$,

$$
L=\left(\begin{array}{rrr}
a_{1,1} & -a_{1,2} & -a_{1,3} \\
-a_{2,1} & a_{2,2} & -a_{2,3} \\
-a_{3,1} & -a_{3,2} & a_{3,3}
\end{array}\right) .
$$

If $L$ is a GPCB matrix, $I=I(L)$ is homogeneous and $V\left(I, t_{i}\right)=\{0\}$ for all $i$ (see Remark 6.6). Suppose that $L$ is not a GPCB matrix, but a GCB matrix. After renumbering the variables, one may suppose that $a_{2,1}=0$. In particular, $a_{3,1}>0$, $a_{1,2}+a_{3,2}>0$, and $b_{2} a_{2,2}=b_{3} a_{2,3}$, so $a_{2,3}>0$. Let $h_{*, 3}$ be the third row of $\operatorname{adj}(L)$, the adjoint matrix of $L$ :

$$
h_{*, 3}=\left(a_{2,2} a_{3,1}, a_{1,1} a_{3,2}+a_{1,2} a_{3,1}, a_{1,1} a_{2,2}\right) .
$$

It follows that $h_{*, 3} \in \mathbb{N}_{+}^{3}$. Set $\mathbf{d}=h_{*, 3} / \operatorname{gcd}\left(h_{*, 3}\right)$. Then $\mathbf{d} \in \mathbb{N}_{+}^{3}, \operatorname{gcd}(\mathbf{d})=1$, and $\mathbf{d} L=0$. Hence $I=I(L)$ is homogeneous. Moreover,

$$
I=\left(t_{1}^{a_{1,1}}-t_{3}^{a_{3,1}}, t_{2}^{a_{2,2}}-t_{1}^{a_{1,2}} t_{3}^{a_{3,2}}, t_{3}^{a_{3,3}}-t_{1}^{a_{1,3}} t_{2}^{a_{2,3}}\right) .
$$

Clearly $\operatorname{rad}\left(I, t_{1}\right)=\mathfrak{m}$ and $\operatorname{rad}\left(I, t_{3}\right)=\mathfrak{m}$. Since $a_{2,3}>0$, it follows that $\operatorname{rad}\left(I, t_{2}\right)=\mathfrak{m}$ too. Thus $V\left(I, t_{i}\right)=\{0\}$ for all $i$.

Proposition 8.5. Let $I=I(L)$ be the $C B$ ideal associated with a $3 \times 3 C B$ matrix $L$. Then the following conditions hold:

(a) $t_{2}^{a_{2,3}} f_{1}+t_{3}^{a_{3,1}} f_{2}+t_{1}^{a_{1,2}} f_{3}=0$ and $t_{3}^{a_{3,2}} f_{1}+t_{1}^{a_{1,3}} f_{2}+t_{2}^{a_{2,1}} f_{3}=0$;

(b) for $\{i, j, k\}=\{1,2,3\}$, then $f_{i}, f_{j}, t_{k}$ is a regular sequence (in any order);

(c) I is either a complete intersection or an almost complete intersection;

(d) I is an unmixed ideal of height 2;

(e) $I$ is a homogeneous lattice ideal and $I=I(\mathcal{L})=\left(I:\left(t_{1} t_{2} t_{3}\right)^{\infty}\right)=\operatorname{Hull}(I)$.

Proof. The proof of (a) follows from a simple check. By Lemma 8.4, $S$ can be graded with $t_{1}, t_{2}, t_{3}$ and $f_{1}, f_{2}, f_{3}$ homogeneous elements of positive degree. Using [29, Proposition 4.2, (c)], one deduces (b). If $I$ is a PCB ideal, by Corollary 6.9, $I$ is an almost complete intersection of height 2. If $I$ is not a PCB ideal, $a_{i, j}=0$ for some $i \neq j$. Using (a), $I$ is generated by two of the three $f_{1}, f_{2}, f_{3}$. In particular, by (b), $I$ is a complete intersection. This proves (c). If $I$ is a PCB ideal, by [30, Remark 4.7 and Proposition 3.3], $I$ is an unmixed ideal of height 2. If $I$ is not a PCB ideal, $a_{i, j}=0$ for some $i \neq j$, by (c), $I$ is a complete intersection, hence unmixed too. This proves (d). By Lemma 8.4, I=I(L) is graded and $V\left(I, t_{i}\right)=\{0\}$ for all $i$. Moreover $I=I(L)$ is an unmixed binomial ideal associated with an integer matrix $L$ (in fact a CB matrix). By Proposition 5.7, $I=I(\mathcal{L})=\left(I:\left(t_{1} t_{2} t_{3}\right)^{\infty}\right)=\operatorname{Hull}(I)$ is a homogeneous lattice ideal.

As a consequence of Theorem 8.2 and Proposition 8.5, we obtain the main result of the section.

TheOREm 8.6. Let $S=K\left[t_{1}, t_{2}, t_{3}\right]$ and let $I$ be an ideal of $S$. Then $I$ is a homogeneous lattice ideal of dimension 1 if and only if $I$ is a CB ideal.

Proof. Suppose that $I$ is a homogeneous lattice ideal of $S$ of dimension 1. By Theorem 8.2, only two cases can occur for $I$. In the first case, $I=\left(f_{1}, f_{2}\right)$, where $f_{1}=t_{1}^{a_{1}}-t_{3}^{c_{3}}$ and $f_{2}=t_{2}^{b_{2}}-t_{1}^{b_{1}} t_{3}^{b_{3}}$, with $0 \leq b_{1} \leq a_{1}$ and $a_{1}, b_{2}, c_{3}>0$. Then $I$ is the CB ideal associated with the CB matrix $L$, where

$$
L=\left(\begin{array}{rrc}
a_{1} & -b_{1} & -a_{1}+b_{1} \\
0 & b_{2} & -b_{2} \\
-c_{3} & -b_{3} & b_{3}+c_{3}
\end{array}\right),
$$

because $f_{3}=-t_{3}^{b_{3}} f_{1}-t_{1}^{a_{1}-b_{1}} f_{2} \in\left(f_{1}, f_{2}\right)$. 
In the second case, $I=\left(f_{1}, f_{2}, f_{3}\right)$, where $f_{1}=t_{1}^{a_{1}}-t_{2}^{a_{2}} t_{3}^{a_{3}}, f_{2}=t_{2}^{b_{2}}-t_{1}^{b_{1}} t_{3}^{b_{3}}$, and $f_{3}=t_{3}^{c_{3}}-t_{1}^{c_{1}} t_{2}^{c_{2}}$, with $0<a_{2}<b_{2}, 0<a_{3}<c_{3}, 0<b_{1}<a_{1}, 0<b_{3}<c_{3}, 0<c_{1}<a_{1}$, and $0<c_{2}<b_{2}$, with $a_{1}=b_{1}+c_{1}, b_{2}=a_{2}+c_{2}$, and $c_{3}=a_{3}+b_{3}$. Then $I$ is the PCB ideal associated with the PCB matrix $L$, where

$$
L=\left(\begin{array}{rrr}
a_{1} & -b_{1} & -c_{1} \\
-a_{2} & b_{2} & -c_{2} \\
-a_{3} & -b_{3} & c_{3}
\end{array}\right) .
$$

Conversely, if $I$ is a CB ideal, $I$ is a graded lattice ideal of dimension 1 by Proposition 8.5.

Next, we show that the homogeneous lattices of rank 2 in $\mathbb{Z}^{3}$ are precisely the lattices generated by the columns of a $\mathrm{CB}$ matrix.

Corollary 8.7. Let $\mathcal{L}$ be a lattice of rank 2 in $\mathbb{Z}^{3}$. Then, $\mathcal{L}$ is homogeneous if and only if $\mathcal{L}$ is generated by the columns of a $C B$ matrix.

Proof. If $\mathcal{L}$ is homogeneous of rank $2, I(\mathcal{L})$ is homogeneous of height 2. By Theorem 8.6, $I(\mathcal{L})$ is a CB ideal. Hence, by Corollary $2.11, \mathcal{L}$ is generated by the columns of a $\mathrm{CB}$ matrix. Conversely, let $\mathcal{L}$ be the lattice generated by the columns of a $3 \times 3$ CB matrix $L$. Clearly $L$ has rank 2 and, by Proposition 8.5, $\mathbf{d} L=0$ for some $\mathbf{d} \in \mathbb{N}_{+}^{3}$. In particular, $\mathcal{L}$ is homogeneous.

In the next result we add a new condition for a GPCB ideal to be a lattice ideal (see Propositions 5.3 and 5.7(b)).

Proposition 8.8. Let $I=I(L)$ be the binomial ideal associated with an $s \times s$ GPCB matrix L. Then $I$ is a lattice ideal if and only if $s \leq 3$ and $I$ is a PCB ideal.

Proof. $(\Rightarrow)$ Assume that $I$ is a lattice ideal. By Proposition $6.1, s \leq 3$. If $s=2$, by Lemma $6.12, I$ is a PCB ideal. If $s=3$, by Theorem $6.3, I$ is a graded lattice ideal. Hence, by Proposition 6.2, I cannot be a complete intersection. Applying Theorem 8.2, we get as in the final paragraph of the proof of Theorem 8.6 that $I$ is a PCB ideal.

$(\Leftarrow)$ Assume that $I$ is a PCB ideal. In particular $I$ is a $\mathrm{CB}$ ideal. If $s=3$, by Theorem 8.6, $I$ is a lattice ideal. If $s=2$, by Lemma $6.12, I$ is a lattice ideal.

As a corollary of Theorem 8.6 we deduce the structure of the hull of a GCB ideal.

Corollary 8.9. Let $I=I(L)$ be the $G C B$ ideal associated with a $3 \times 3 G C B$ matrix $L$. Then $I(\mathcal{L})$ is a $C B$ ideal.

Proof. By Lemma 8.4, $I$ is homogeneous with $V\left(I, t_{i}\right)=\{0\}$ for all $i$. Therefore, $I(\mathcal{L})$ is a homogeneous lattice ideal of dimension 1 (see Proposition 5.7). Thus, by Theorem 8.6, $I(\mathcal{L})$ is a CB ideal.

We deduce a method to find a generating set for the hull of a GCB ideal.

Procedure 8.10. Given a $3 \times 3$ GCB matrix $L$,

(a) find a $C B$ matrix $M$ such that $\mathcal{M}=\mathcal{L}$, where $\mathcal{M}$ and $\mathcal{L}$ are the lattices of $\mathbb{Z}^{3}$ spanned by the columns of $M$ and $L$, respectively. Equivalently, find a $C B$ matrix $M$ and $a 3 \times 3$ integer matrix $Q$ with $\operatorname{det}(Q)=1$, such that $L Q=M$;

(b) by Proposition 8.5, $I(M)$ is a lattice ideal and $I(M)=I(\mathcal{M})$. Hence $I(\mathcal{L})=$ $I(M)$.

We illustrate this method with some examples.

Example 8.11. Let $I=\left(f_{1}, f_{2}, f_{3}\right)=\left(t_{1}^{4}-t_{2} t_{3}, t_{2}^{3}-t_{1}^{5} t_{3}, t_{3}^{3}-t_{1}^{3} t_{2}\right)$ be the GPCB ideal associated with the GPCB matrix

$$
L=\left(\begin{array}{rrr}
4 & -5 & -3 \\
-1 & 3 & -1 \\
-1 & -1 & 3
\end{array}\right) .
$$

Copyright $@$ by SIAM. Unauthorized reproduction of this article is prohibited. 
Here $L \mathbf{b}^{\top}=0$ with $\mathbf{b}=(2,1,1)$. We have $(-1,2,-2)=(4,-1,-1)+(-5,3,-1) \in \mathcal{L}$. Therefore $\mathcal{L}=\langle(4,-1,-1),(-1,2,-2),(-3,-1,3)\rangle$. Take

$$
M=\left(\begin{array}{rrr}
4 & -1 & -3 \\
-1 & 2 & -1 \\
-1 & -2 & 3
\end{array}\right)
$$

which is a PCB matrix. Hence $I(\mathcal{L})=I(\mathcal{M})=I(M)=\left(t_{1}^{4}-t_{2} t_{3}, t_{2}^{2}-t_{1} t_{3}^{2}, t_{3}^{3}-t_{1}^{3} t_{2}\right)$. In this example, the hull of a GPCB ideal is a PCB ideal.

Example 8.12. Let $I=\left(f_{1}, f_{2}, f_{3}\right)=\left(t_{1}^{4}-t_{2}^{2} t_{3}, t_{2}^{3}-t_{1} t_{3}, t_{3}-t_{1} t_{2}\right)$ be the GPCB ideal associated with the GPCB matrix

$$
L=\left(\begin{array}{rrr}
4 & -1 & -1 \\
-2 & 3 & -1 \\
-1 & -1 & 1
\end{array}\right)
$$

Here $L \mathbf{b}^{\top}=0$ with $\mathbf{b}=(2,3,5)$. Let $Q$ and $M$ be the $3 \times 3$ integer matrices

$$
Q=\left(\begin{array}{lll}
1 & 1 & 0 \\
1 & 2 & 0 \\
2 & 2 & 1
\end{array}\right), \operatorname{det}(Q)=1 ; M=\left(\begin{array}{rrr}
1 & 0 & -1 \\
-1 & 2 & -1 \\
0 & -1 & 1
\end{array}\right), \text { a CB matrix; } L Q=M
$$

Hence $I(\mathcal{L})=I(\mathcal{M})=I(M)=\left(t_{1}-t_{2}, t_{2}^{2}-t_{3}, t_{3}-t_{1} t_{2}\right)$. In this example, the hull of a GPCB ideal is a CB ideal.

Example 8.13. Let $\mathcal{L}=\langle(-2,4,-2),(-2,-3,4)\rangle$, which is a rank 2 homogeneous lattice with respect to the vector $(5,6,7)$. Thus the lattice ideal $I(\mathcal{L})$ of $\mathcal{L}$ is a graded lattice ideal of dimension 1 . By Theorem $8.2, I(\mathcal{L})$ is generated by a full set of critical binomials and, by Theorem 8.6, $I(\mathcal{L})$ is a CB ideal (here a PCB ideal). Concretely,

$$
I(\mathcal{L})=\left(\left(t_{2}^{4}-t_{1}^{2} t_{3}^{2}, t_{1}^{2} t_{2}^{3}-t_{3}^{4}\right):\left(t_{1} t_{2} t_{3}\right)^{\infty}\right)=\left(t_{1}^{4}-t_{2} t_{3}^{2}, t_{2}^{4}-t_{1}^{2} t_{3}^{2}, t_{1}^{2} t_{2}^{3}-t_{3}^{4}\right) .
$$

To obtain the above generating set one may "complete" the two generators of $\mathcal{L}$ to a $\mathrm{CB}$ (in fact a $\mathrm{PCB}$ ) matrix $M$, namely,

$$
M=\left(\begin{array}{rrr}
4 & -2 & -2 \\
-1 & 4 & -3 \\
-2 & -2 & 4
\end{array}\right)
$$

and apply Procedure 8.10. The degree of $S / I$ is 14 . If $K=\mathbb{Q}$, then $I=\mathfrak{p}_{1} \cap \mathfrak{p}_{2}$, where $\mathfrak{p}_{1}, \mathfrak{p}_{2}$ are prime ideals of degree 7 .

Example 8.14. Let $\mathcal{L}=\langle(2,-1,-1),(-3,1,-1)\rangle$, which is a rank 2 nonhomogeneous lattice. The lattice ideal $I(\mathcal{L})$ of $\mathcal{L}$ is a nongraded lattice ideal of height 2. By Theorem 8.6, $I(\mathcal{L})$ cannot be a CB ideal. Concretely,

$$
I(\mathcal{L})=\left(\left(t_{1}^{2}-t_{2} t_{3}, t_{2}-t_{1}^{3} t_{3}\right):\left(t_{1} t_{2} t_{3}\right)^{\infty}\right)=\left(t_{1}^{2}-t_{2} t_{3}, t_{1} t_{3}^{2}-1\right) .
$$

If we apply Corollary 4.10 with $v_{1}=-2, v_{2}=-5$, and $v_{3}=1$, we get that $I(\mathcal{L})$ has degree 6 .

Acknowledgments. The authors would like to thank the referees for their careful reading of the paper and for the improvements that they suggested. 


\section{REFERENCES}

[1] C. Alfaro and C. E. Valencia, On the sandpile group of the cone of a graph, Linear Algebra Appl., 436 (2012), pp. 1154-1176.

[2] J. Bang-Jensen and G. Gutin, Digraphs. Theory, Algorithms and Applications, Springer Monogr. Math., 2nd ed., Springer, London, 2006.

[3] A. Berman and R. J. Plemmons, Nonnegative matrices in the mathematical sciences, Classics Appl. Math. 9, SIAM, Philadelphia, 1994.

[4] N. Biggs, Algebraic Graph Theory, Cambridge University Press, Cambridge, 1993.

[5] W. Bruns and J. Herzog, Cohen-Macaulay Rings, Rev. ed., Cambridge University Press, Cambridge, 1997.

[6] W. Bruns and B. Ichim, Normaliz 2.0, Computing Normalizations of Affine Semigroups, http://www.math.uos.de/normaliz (2008).

[7] B. W. Char, K. G. Geddes, G. H. Gonnet, and S. M. Watt, Maple V Language Reference Manual, Springer-Verlag, Berlin, 1991.

[8] H. Corrales and C. E. Valencia, On the critical ideals of graphs, Linear Algebra Appl., 439 (2013), pp. 3870-3892.

[9] D. Cox, J. Little, and D. O'Shea, Ideals, Varieties, and Algorithms, Springer-Verlag, New York, 1992.

[10] D. Eisenbud And B. Sturmfels, Binomial ideals, Duke Math. J., 84 (1996), pp. 1-45.

[11] V. Ene And J. Herzog, Gröbner Bases in Commutative Algebra, Grad. Stud. Math. 130, AMS, Providence, RI, 2012.

[12] C. Escobar, J. Martínez-Bernal, and R. H. Villarreal, Relative volumes and minors in monomial subrings, Linear Algebra Appl., 374 (2003), pp. 275-290.

[13] R. Gilmer, Commutative Semigroup Rings, Chicago Lectures in Math., University of Chicago Press, Chicago, 1984.

[14] C. Godsil and G. Royle, Algebraic Graph Theory, Grad. Texts in Math. 207, Springer, New York, 2001.

[15] G. M. Greuel and G. Pfister, A Singular Introduction to Commutative Algebra, 2nd extended ed., Springer, Berlin, 2008.

[16] J. Herzog, Generators and relations of Abelian semigroups and semigroup rings, Manuscripta Math., 3 (1970), pp. 175-193.

[17] N. Jacobson, Basic Algebra I, 2nd ed., W. H. Freeman, New York, 1996.

[18] T. Kahle and E. Miller, Decompositions of Commutative Monoid Congruences and Binomial Ideals, preprint, arXiv:1107.4699v3, 2012.

[19] E. Kunz, Introduction to Commutative Algebra and Algebraic Geometry, Birkhäuser, Boston, 1985.

[20] H. H. López and R. H. Villarreal, Complete intersections in binomial and lattice ideals, Internat. J. Algebra Comput., 23 (2013), pp. 1419-1429.

[21] H. H. López and R. H. Villarreal, Computing the degree of a lattice ideal of dimension one, J. Symbolic Comput., http://dx.doi.org/10.1016/j.jsc.2014.01.002 .

[22] D. Lorenzini, Smith normal form and Laplacians, J. Combin. Theory Ser. B, 98 (2008), pp. 1271-1300.

[23] M. Manjunath and B. Sturmfels, Monomials, binomials and Riemann-Roch, J. Algebraic Combin., 37 (2013), pp. 737-756.

[24] H. Matsumura, Commutative Algebra, Benjamin-Cummings, Reading, MA, 1980.

[25] H. Matsumura, Commutative Ring Theory, Cambridge Stud. Adv. Math. 8, Cambridge University Press, Cambridge, 1986.

[26] R. MerRis, Laplacian matrices of graphs: A survey, Linear Algebra Appl., 197/198 (1994), pp. 143-176.

[27] E. Miller And B. Sturmfels, Combinatorial Commutative Algebra, Grad. Texts in Math. 227, Springer, New York, 2004.

[28] M. Newman, Integral Matrices, Pure Appl. Math. 45, Academic Press, New York, 1972.

[29] L. O'Carroll and F. Planas-Vilanova, Ideals of Herzog-Northcott type, Proc. Edinburgh Math. Soc. (2), 54 (2011), pp. 161-186.

[30] L. O'Carroll and F. Planas-Vilanova, The primary components of positive critical binomial ideals, J. Algebra, 373 (2013), pp. 392-413.

[31] I. Peeva And B. Sturmfels, Syzygies of codimension 2 lattice ideals, Math. Z., 229 (1998), pp. 163-194.

[32] D. Perkinson, J. Perlman, and J. Wilmes, Primer for the Algebraic Geometry of Sandpiles, preprint, arXiv:1112.6163v2, 2011.

Copyright (C) by SIAM. Unauthorized reproduction of this article is prohibited. 
[33] C. Rentería, A. Simis, and R. H. Villarreal, Algebraic methods for parameterized codes and invariants of vanishing ideals over finite fields, Finite Fields Appl., 17 (2011), pp. 81-104.

[34] B. Sturmfels, Gröbner Bases and Convex Polytopes, Univ. Lecture Ser. 8, AMS, Providence, RI, 1996.

[35] W. V. Vasconcelos, Computational Methods in Commutative Algebra and Algebraic Geometry, Springer-Verlag, Berlin, 1998.

[36] R. H. Villarreal, Monomial Algebras, Monogr. Textbooks Pure Appl. Math. 238, Marcel Dekker, New York, 2001.

[37] R. H. Villarreal, Monomial algebras and polyhedral geometry, in Handbook of Algebra, Vol. 3, M. Hazewinkel, ed., Elsevier, Amsterdam, 2003, pp. 257-314.

[38] O. Zariski and P. Samuel, Commutative Algebra, Vol. II, Grad. Texts Math. 29, SpringerVerlag, New York, 1975.

Copyright (c) by SIAM. Unauthorized reproduction of this article is prohibited. 